\title{
Rigid surface operators
}

\author{
Sergei Gukov ${ }^{1,2,4}$ and Edward Witten ${ }^{3}$
}

${ }^{1}$ Department of Physics, University of California, Santa Barbara, CA 93106, USA

${ }^{2}$ School of Mathematics, Institute for Advanced Study, Princeton, NJ 08540, USA

gukov@theory.caltech.edu

${ }^{3}$ School of Natural Sciences, Institute for Advanced Study, Princeton, NJ 08540, USA

${ }^{4}$ On leave from California Institute of Technology.

\begin{abstract}
Surface operators in gauge theory are analogous to Wilson and 't Hooft line operators except that they are supported on a two-dimensional surface rather than a one-dimensional curve. In a previous paper, we constructed a certain class of half-BPS surface operators in $\mathcal{N}=4$ super Yang-Mills theory, and determined how they transform under $S$-duality. Those surface operators depend on a relatively large number of freely adjustable parameters. In the present paper, we consider the opposite case of half-BPS surface operators that are "rigid" in the sense that they do not depend on any parameters at all. We present some simple constructions of rigid half-BPS surface operators and attempt to determine how they transform under duality. This attempt is only partially successful, suggesting that our constructions are not the whole story. The partial match suggests interesting connections with quantization. We discuss some possible refinements and some string theory constructions which might lead to a more complete picture.
\end{abstract}

e-print archive: http://lanl.arXiv.org/abs/hep-th//0804.1561 


\section{Contents}

$\begin{array}{llr}1 & \text { Introduction } & \mathbf{9 0}\end{array}$

$\begin{array}{lll}1.1 & \text { Organization of the paper } & 92\end{array}$

$\begin{array}{lll}2 & \text { Rigid surface operators } & 93\end{array}$

$\begin{array}{lll}2.1 & \text { Review } & 93\end{array}$

$2.2 \quad$ Limit for $\alpha, \beta, \gamma \rightarrow 0 \quad 95$

$\begin{array}{lll}2.2 .1 & \text { The monodromy } & 96\end{array}$

$\begin{array}{lll}\text { 2.2.2 Counting dimensions } & 99\end{array}$

2.2.3 More general conjugacy classes 100

2.3 Searching for rigid surface operators 103

$\begin{array}{lll}\text { 2.3.1 Some examples } & 105\end{array}$

2.3.2 Computing the dimension of a unipotent orbit 106

2.3.3 Strongly rigid orbits in orthogonal and symplectic groups $\quad 107$

2.4 Strongly rigid semisimple orbits 110

2.4.1 Rigid semisimple surface operators $\quad 115$

2.5 Combining the two constructions 116

3 Alternative point of view 118

$\begin{array}{lll}3.1 & \text { Coupling to sigma models } & 118\end{array}$

$\begin{array}{lll}3.2 & \text { An example } & 120\end{array}$

$\begin{array}{lll}3.3 & \text { Another example } & 122\end{array}$

3.3.1 Cover of a unipotent orbit 125 
3.3.2 Semisimple surface operators

3.4 Rigid surface operator for the dual group

3.4.1 Duality conjecture

3.5 Minimal surface operators

4 Fingerprints of surface operators

4.1 Invariant polynomials

4.1.1 The dimension

4.2 Polar polynomials

4.3 Center versus topology

5 Duality for strongly rigid surface operators

5.1 Duality for $G=\operatorname{SO}(5)$ and ${ }^{L} G=\operatorname{Sp}(4)$

5.2 Duality for $G=\operatorname{SO}(8)$

5.3 Duality for $G=\operatorname{SO}(7)$ and ${ }^{L} G=\operatorname{Sp}(6)$

5.4 Duality for $G=\operatorname{SO}(9)$ and ${ }^{L} G=\operatorname{Sp}(8)$

6 More examples

6.1 Special rigid orbits

6.2 Dualities involving rigid semisimple orbits

$7 \quad$ Duality and quantization

8 Stringy constructions of rigid surface operators 
Appendix A Rigid nilpotent orbits for exceptional groups 169

Appendix B Orthogonal and symplectic Lie algebras and duality

\section{References}

\section{Introduction}

The familiar examples of non-local operators in four-dimensional gauge theory include line operators, such as Wilson and 't Hooft operators, supported on a one-dimensional curve $L$ in the space-time manifold $M$. While a Wilson operator labeled by a representation $R$ of the gauge group $G$ can be defined by modifying the measure in the path integral, namely by inserting a factor

$$
W_{R}(L)=\operatorname{Tr}_{R} \operatorname{Hol}_{L}(A)=\operatorname{Tr}_{R}\left(P \exp \oint_{L} A\right),
$$

an 't Hooft operator is defined by modifying the space of fields over which one performs the path integral.

Similarly, a surface operator in four-dimensional gauge theory is an operator supported on a two-dimensional submanifold $D \subset M$ in the space-time manifold $M$. Although in this paper we mainly take $M=\mathbb{R}^{4}$ and $D=\mathbb{R}^{2}$, the constructions are local and one might consider more general space-time four-manifolds $M$ and embedded surfaces $D$. In general, surface operators do not admit a simple "electric" description analogous to the definition of Wilson lines, and should be defined, like 't Hooft operators, by modifying the domain of integration in the path integral, that is by requiring the gauge field $A$ (and, possibly, other fields) to have prescribed singularities along $D$.

Four-dimensional gauge theories admit surface operators, and in the supersymmetric case, they often admit supersymmetric surface operators, that is, surface operators that preserve some of the supersymmetry. In this paper, we consider $\mathcal{N}=4$ super Yang-Mills theory in four dimensions, the 
maximally supersymmetric case. This theory has many remarkable properties, including electric-magnetic duality, and has been extensively studied in the context of string dualities, in particular in the AdS/CFT correspondence [1]. It also has a rich spectrum of non-local operators, including supersymmetric Wilson and 't Hooft operators that play an important role in many applications, as well as supersymmetric surface operators and domain walls.

A half-supersymmetric or half-BPS Wilson operator is determined by discrete data, namely the choice of a representation of the gauge group $G$. Similarly, a half-BPS 't Hooft operator is determined by discrete data. In contrast, the half-BPS surface operators that we constructed in previous work [2] depend on freely adjustable parameters, typically quite a few of them. Much of their interest actually comes from the dependence on these parameters.

As will become clear, the problem of describing all half-BPS surface operators in $\mathcal{N}=4$ super Yang-Mills theory is rather involved. In this paper, we will consider the opposite case from what was considered in [2]: surface operators that depend on no continuously variable parameters at all. We call these rigid surface operators.

It is purely for simplicity that we consider only maximally supersymmetric or half-BPS surface operators. In the case of line operators, in addition to the half-BPS Wilson and 't Hooft operators, there are many more $\frac{1}{4}$-BPS line operators; their analysis is very interesting but is much more complex than the half-BPS case, as shown in [3]. Surface operators with reduced supersymmetry are probably also interesting, but harder to study.

In addition to being rigid, the surface operators that we consider here are in a certain sense minimal or irreducible. They do not have any extra fields supported on the surface. This notion is clarified in Section 3.5; in the meanwhile, we simply remark that our surface operators are related to individual orbits of the gauge group $G$ (or rather its complexification), and this leads to minimality.

Finally, rigid surface operators are probably automatically conformally invariant. They must be scale-invariant, or a scale transformation would introduce a free parameter. In local quantum field theory, scale invariance usually implies conformally invariance. Our constructions will be manifestly conformally invariant at the classical level. Quantum conformal invariance can probably be argued along the lines of [4], and is manifest for some of our surface operators in the string theory construction of Section 8. $\mathcal{N}=4$ super Yang-Mills theory also has (non-rigid) half-BPS surface operators that are not conformally invariant [5]. 


\subsection{Organization of the paper}

In Section 2, after a brief review of the surface operators considered in [2], we describe two constructions of rigid surface operators. Some further refinements leading to additional rigid surface operators are described in Section 3.

$S$-duality must transform rigid surface operators of $\mathcal{N}=4$ super YangMills theory with gauge group $G$ to similar operators in the same theory with the dual gauge group ${ }^{L} G$. Aiming to understand this, we describe in Section 4 some properties of surface operators that are computable and should be invariant under electric-magnetic duality or should transform in a known way.

In Section 5, we attempt to use this information to determine, in examples, how our surface operators transform under duality. In doing this, we concentrate on orthogonal and symplectic gauge groups of small rank. A simple example involving unitary groups is also discussed in Section 3.4. We omit exceptional groups, which are more complicated. It is especially interesting to consider the dual pairs of groups $G=\mathrm{SO}(2 n+1)$ and ${ }^{L} G=\operatorname{Sp}(2 n)$, whose Lie algebras are not isomorphic. In carrying out this analysis, we do find some interesting examples of what appear to be dual pairs of surface operators, but we are not able to get a complete duality conjecture. It is quite likely that our constructions of rigid surface operators are in need of some further refinement. There may be a relation to the construction in.

The remainder of the paper is devoted to some attempts at a more systematic understanding. In Section 6, we try to be more systematic, at least for certain families of rigid surface operators, in orthogonal and symplectic gauge groups of any rank. In Section 6.1, we argue that the mathematical theory of special unipotent conjugacy classes $[6,7]$ provides the right framework for a duality conjecture for a certain family of surface operators. In Section 6.2, we make analogous proposals for other families of surface operators. This discussion is somewhat similar to a relation between conjugacy classes defined in [8, Section 13.3]. In Section 7, we make a general conjecture about how the conjugacy class associated with a rigid surface operator transforms under duality. Finally, in Section 8, we describe string theory constructions of some of the rigid surface operators of Section 2.

The paper contains two appendices. In Appendix A, we describe rigid nilpotent orbits for exceptional groups which, together with the material of Section 2, can be used to study rigid surface operators in super Yang-Mills theories with exceptional gauge groups. In Appendix B, we review the root 
systems and matrix realizations of the Lie algebras $\mathfrak{s o}(2 N+1)$ and $\mathfrak{s p}(2 N)$. In particular, we identify the invariant polynomials of the Higgs field in dual theories with gauge groups $G=\mathrm{SO}(2 n+1)$ and ${ }^{L} G=\mathrm{Sp}(2 n)$ which play an important role in identifying dual pairs of rigid surface operators.

\section{Rigid surface operators}

\subsection{Review}

To keep this paper self-contained, we begin with a brief review of the surface operators constructed in [2]. We consider $\mathcal{N}=4$ super Yang-Mills theory on $\mathbb{R}^{4}$, with coordinates $x^{0}, x^{1}, x^{2}, x^{3}$. The support $D$ of the surface operator will be a copy of $\mathbb{R}^{2}$ at $x^{2}=x^{3}=0$. The supersymmetry preserved by the surface operator is $(4,4)$ supersymmetry in the two-dimensional sense. We recall that the vector multiplet of $(4,4)$ supersymmetry in two dimensions consists of a gauge field and four scalars in the adjoint representation (plus fermions). Accordingly, components $A_{0}, A_{1}$ of the four-dimensional gauge field plus four of the six scalars of $\mathcal{N}=4$ super Yang-Mills theory transform in a vector multiplet of two-dimensional $(4,4)$ supersymmetry. The "normal" components $A_{2}$ and $A_{3}$ of the gauge field transform in a hypermultiplet of the unbroken supersymmetry, along with two of the scalars. It is convenient to denote those two scalars as $\phi_{2}$ and $\phi_{3}$.

Surface operators were defined in [2] by postulating a suitable singular behavior of the hypermultiplets, that is the fields $A_{2}, A_{3}, \phi_{2}, \phi_{3}$, at $x^{2}=x^{3}=0$. Of course, the singularity must be chosen to be compatible with supersymmetry. The condition for supersymmetry is that $A=$ $A_{2} d x^{2}+A_{3} d x^{3}$ and $\phi=\phi_{2} d x^{2}+\phi_{3} d x^{3}$ must obey certain equations that are known as Hitchin's equations [9]. Hitchin's equations are equations in the $x^{2}-x^{3}$ plane that can be written as follows:

$$
\begin{aligned}
& F_{A}-\phi \wedge \phi=0, \\
& d_{A} \phi=0, \quad d_{A} \star \phi=0 .
\end{aligned}
$$

Originally, these equations were obtained in [9] as the dimensional reduction of the self-dual Yang-Mills equations from four to two dimensions; $\phi$ simply arises as the components of the gauge field in the two hidden dimensions. (This approach is natural if one considers $\mathcal{N}=4$ super Yang-Mills theory to arise by dimensional reduction from ten dimensions.) This interpretation of Hitchin's equations makes it clear they are associated with unbroken supersymmetry. 
To define a supersymmetric surface operator, one picks a solution of Hitchin's equations with a singularity along $D$, and one requires that quantization of $\mathcal{N}=4$ super Yang-Mills theory should be carried out for fields with precisely this kind of singularity. For the surface operator to be superconformal, the singularity must be scale-invariant. In addition, it is natural to look for surface operators that are invariant under rotations of the $x^{2}-x^{3}$ plane. If we set $x^{2}+\mathrm{i} x^{3}=r \mathrm{e}^{\mathrm{i} \theta}$, then the most general possible rotationinvariant ansatz is

$$
\begin{aligned}
& A=a(r) \mathrm{d} \theta+f(r) \frac{\mathrm{d} r}{r}, \\
& \phi=b(r) \frac{\mathrm{d} r}{r}-c(r) \mathrm{d} \theta .
\end{aligned}
$$

Setting $f(r)=0$ by a gauge transformation and introducing a new variable $s=-\ln r$, we can write the supersymmetry equations (2.1) in the form of Nahm's equations:

$$
\begin{aligned}
& \frac{\mathrm{d} a}{\mathrm{~d} s}=[b, c], \\
& \frac{\mathrm{d} b}{\mathrm{~d} s}=[c, a], \\
& \frac{\mathrm{d} c}{\mathrm{~d} s}=[a, b] .
\end{aligned}
$$

A conformally invariant solution is invariant under scalings of $r$ and therefore is independent of $s$. (As we discuss later, solutions that are not quite conformally invariant can also be used to construct conformally invariant surface operators.) So the most general conformally invariant solution is obtained by setting $a, b, c$ to constant elements $\alpha, \beta, \gamma$ of the Lie algebra $\mathfrak{g}$ of $G$. The equations imply that $\alpha, \beta$, and $\gamma$ must commute, so we can conjugate them to the Lie algebra $\mathfrak{t}$ of a maximal torus $\mathbb{T}$ of $G$. The resulting singular solution of Hitchin's equations then takes the simple form

$$
\begin{aligned}
& A=\alpha \mathrm{d} \theta, \\
& \phi=\beta \frac{\mathrm{d} r}{r}-\gamma \mathrm{d} \theta .
\end{aligned}
$$

Hitchin's equations with a singularity of this form were first studied mathematically in [10].

Roughly speaking, surface operators were defined in [2] by requiring that the fields have a singularity of this kind, with specified values ${ }^{1}$ of $\alpha, \beta$, and

\footnotetext{
${ }^{1}$ If instead of specifying the values of $\alpha, \beta$, and $\gamma$, we treat them as dynamical fields, we get a non-minimal surface operator, in the sense of Section 3.5.
} 
$\gamma$. More exactly, to study $\mathcal{N}=4$ super Yang-Mills theory in the presence of the surface operator, one performs the path integral (or one quantizes) in a space of fields that take the form given in equation (2.4) modulo terms that are less singular than $1 / r$.

There are two important caveats. First, it turns out that one can add an additional parameter $\eta$, also $\mathfrak{t}$-valued. $\eta$ is a sort of two-dimensional theta angle and plays an important role because it transforms into $\alpha$ under duality. (For rigid surface operators, $\eta$ at most has only a discrete analog.) Second, to quantize in the presence of the singularity described in (2.4), one should divide only by gauge transformations that, along the locus $D$ of the singularity, take values in the subgroup of $G$ that commutes with $\alpha, \beta$, and $\gamma($ and $\eta$ ). Generically, this subgroup is the maximal torus $\mathbb{T}$. But in general, it may be any subgroup $\mathbb{L}$ of $G$ that contains $\mathbb{T}$. Such a subgroup is called a Levi subgroup. In studying a surface operator of this type, we regard the choice of $\mathbb{L}$ as part of the definition. Having chosen $\mathbb{L}$, we pick $\alpha, \beta, \gamma$, and $\eta$ to be an $\mathbb{L}$-regular quadruple, meaning that the subgroup of $G$ that commutes with all four of them is precisely $\mathbb{L}$. Then, to calculate Yang-Mills observables in the presence of the surface operator, we perform a path integral over fields with the indicated type of singularity, dividing by gauge transformations that along $D$ are $\mathbb{L}$-valued. This gives a surface operator that varies smoothly with $\alpha, \beta, \gamma, \eta$ as long as those parameters form an $\mathbb{L}$-regular quadruple. But when the parameters are varied so that the unbroken group becomes a larger group $\mathbb{L}^{\prime}$, a singularity emerges. In a sense, the residue of this singularity is a surface operator that can be constructed in the same way, but starting with $\mathbb{L}^{\prime}$ rather than $\mathbb{L}$. One of the main ideas in [2] was to study the monodromies in the space of $\mathbb{L}$-regular parameters.

\subsection{Limit for $\alpha, \beta, \gamma \rightarrow 0$}

As a preliminary to discussing rigid surface operators, we will consider what happens to the above construction in the limit that $\alpha, \beta, \gamma \rightarrow 0$. To keep things simple, we begin with the case $G=\mathrm{SU}(2)$. For more detail on the following, see [2, Section 3.3].

The naive idea is that the singularity of $A$ and $\phi$ is linear in $\alpha, \beta$, and $\gamma$, so that if we set $\alpha, \beta, \gamma$ to zero, there is no singularity and no surface operator. However, as we have already noted, the definition of the surface operator is that $A$ and $\phi$ have singularities proportional to $\alpha, \beta, \gamma$ modulo terms that are less singular than $1 / r$. Generically, for $\alpha, \beta, \gamma \rightarrow 0$, we should not conclude that $A$ and $\phi$ are non-singular, but only that they are less singular than $1 / r$. In fact, Hitchin's equations do have a rotationally symmetric solution that 
is singular at $r=0$ but less singular than $1 / r$. The Nahm equations $(2.3)$ are solved with

$$
a=-\frac{t_{1}}{s+1 / f}, \quad b=-\frac{t_{2}}{s+1 / f}, \quad c=-\frac{t_{3}}{s+1 / f},
$$

where $t_{1}, t_{2}$, and $t_{3}$ are elements of the Lie algebra $\mathfrak{g}$, which satisfy the usual $\mathfrak{s u}(2)$ commutation relations, $\left[t_{1}, t_{2}\right]=t_{3}$, etc. Moreover, $f$ is an arbitrary non-negative constant. Since we are taking $G=\mathrm{SU}(2)$, the matrices $t_{i}$, if non-zero, correspond to the two-dimensional representation of $\mathrm{SU}(2)$.

Because of the factor of $-1 / s=1 / \ln r$, this solution is less singular at $r=0$ than the solutions considered before in which $a, b, c$ are set to commuting constants $\alpha, \beta, \gamma$. A surface operator with non-zero $\alpha, \beta, \gamma$ converges for $\alpha, \beta, \gamma \rightarrow 0$ to one that is characterized by the statement that the singularity at $r=0$ looks like the solution of equation (2.5), for some $f$. (We also allow the limiting case $f=0$, in which there is no singularity.) Any choice of $f$ would spoil conformal invariance. But it is not natural to make a choice of $f$, because the derivative of $A$ and $\phi$ with respect to $f$ is square integrable. So the surface operator that we get from the ansatz (2.5), with $f$ allowed to fluctuate, is actually conformally invariant.

A convenient way to describe this surface operator is to say that the fields behave near $r=0$ as

$$
\begin{aligned}
& A=\frac{t_{1} \mathrm{~d} \theta}{\ln r}+\cdots, \\
& \phi=\frac{t_{2} \mathrm{~d} r}{r \ln r}-\frac{t_{3} \mathrm{~d} \theta}{\ln r}+\cdots,
\end{aligned}
$$

where the ellipses refer to terms that are less singular (at most of order $\left.1 / r \ln ^{2} r\right)$ at $r=0$.

Concretely, a generic field with the singularity determined by $\alpha, \beta, \gamma$ has (in a basis in which $\alpha, \beta, \gamma$ are diagonal) off-diagonal terms that are singular, but less singular than $1 / r$. For $\alpha, \beta, \gamma \rightarrow 0$, a sequence of such solutions can converge to the one given in equation (2.5). Such a sequence can also converge to a non-singular solution (corresponding to $f=0$ ), but that is non-generic.

\subsubsection{The monodromy}

The following considerations give a useful picture of what is happening. The complex-valued flat connection $\mathcal{A}=A+\mathrm{i} \phi$ is invariant under part of 
the supersymmetry preserved by the surface operator. Hence the conjugacy class of the monodromy

$$
U=P \exp \left(-\int_{\ell} \mathcal{A}\right)
$$

is a supersymmetric observable. Here $\ell$ is a contour surrounding the singularity. Hitchin's equations imply that the curvature of $\mathcal{A}$, namely $\mathcal{F}=$ $d \mathcal{A}+\mathcal{A} \wedge \mathcal{A}$, is equal to zero. So if Hitchin's equations are obeyed, then the conjugacy class of $U$ is invariant under deformations of $\ell$. Of course, $U$ is an element of $G_{\mathbb{C}}$, the complexification of $G$.

In general, in quantum theory, the fields fluctuate and Hitchin's equations are only obeyed near the singularity (where they are imposed as a boundary condition). However, the conjugacy class of $U$ is independent of $\ell$ as an observable in a suitable chiral algebra, defined using some of the supersymmetries, since $\mathcal{F}$ vanishes in that chiral algebra. Alternatively, one can simply define the conjugacy class of $U$ for the limiting case that $\ell$ is a small loop surrounding the singularity.

So let us compute the conjugacy class of $U$ for the surface operators that were described above. For a generic surface operator with parameters $\alpha, \beta, \gamma$, we set $\xi=\alpha-\mathrm{i} \gamma$. Then $\mathcal{A}=\xi \mathrm{d} \theta$, and the monodromy is hence

$$
U=\exp (-2 \pi \xi) .
$$

This is independent of the choice of $\ell$.

On the other hand, for solution (2.5), we find $\mathcal{A}=-\mathrm{d} \theta\left(t_{1}-\mathrm{i} t_{3}\right) /(s+1 / f)$. If we take $\ell$ to be the circle $s=s_{1}$, the monodromy comes out to be

$$
U^{\prime}=\exp \left(-2 \pi\left(t_{1}-\mathrm{i} t_{3}\right) /\left(s_{1}+1 / f\right)\right) .
$$

At first sight, it is not obvious that the conjugacy class of $U^{\prime}$ is independent of $s_{1}$, as it should be. What saves the day is that $t_{1}-\mathrm{i} t_{3}$ is nilpotent, because of the commutation relation

$$
\left[\mathrm{i} t_{2}, t_{1}-\mathrm{i} t_{3}\right]=t_{1}-\mathrm{i} t_{3} .
$$

In a form of the two-dimensional representation of $\mathrm{SU}(2)$, with $t_{2}$ being diagonal, $t_{1}-\mathrm{i} t_{3}$ is lower triangular. Thus $U^{\prime}$ takes the form

$$
U^{\prime}=\left(\begin{array}{cc}
1 & 0 \\
w & 1
\end{array}\right)
$$

for some $w$. 
The conjugacy class of $U^{\prime}$ is independent of $w$, as long as $w$ is non-zero, because $w$ can be changed by conjugating $U^{\prime}$ by a diagonal matrix. Now let us reconsider the monodromy (2.8) of the surface operator with $\alpha, \gamma \neq 0$. If $\xi \neq 0$, then $\xi$ can be diagonalized with eigenvalues $\pm \xi_{0}$. $U$ can also be diagonalized, with eigenvalues $\exp \left( \pm 2 \pi \xi_{0}\right)$ :

$$
U=\left(\begin{array}{cc}
\exp \left(-2 \pi \xi_{0}\right) & 0 \\
0 & \exp \left(2 \pi \xi_{0}\right)
\end{array}\right)
$$

As long as $\xi_{0} \neq 0$, this matrix is conjugate to

$$
U_{w}=\left(\begin{array}{cc}
\exp \left(-2 \pi \xi_{0}\right) & 0 \\
w & \exp \left(2 \pi \xi_{0}\right)
\end{array}\right)
$$

so it does not matter if $w$ is zero or not. In fact, $U$ can be transformed to $U_{w}$ by conjugation by a lower triangular matrix

$$
\left(\begin{array}{ll}
1 & 0 \\
* & 1
\end{array}\right)
$$

But if $\xi_{0}=0$, then of course, the conjugacy class of $U_{w}$ does depend on whether $w$ vanishes or not.

Let $\mathfrak{C}_{\xi}$ be the conjugacy class in $\operatorname{SL}(2, \mathbb{C})$ that contains the element $U=$ $\exp (-2 \pi \xi)$, with generic $\xi$. Then $\mathfrak{C}_{\xi}$ is of complex dimension two. Indeed, $U$ commutes only with a one-parameter subgroup of diagonal matrices, so its orbit in the three dimensional group $\operatorname{SL}(2, \mathbb{C})$ is two dimensional. Similarly, the lower triangular matrix $U^{\prime}$ commutes only with the one-parameter group of lower-triangular matrices, so it lies in a two-dimensional conjugacy class $\mathfrak{C}^{\prime}$. The limit of the conjugacy class $\mathfrak{C}_{\xi}$ for $\xi \rightarrow 0$ is $\mathfrak{C}^{\prime}$ (or more precisely its closure, as we note in a moment). It is not the conjugacy class $\mathfrak{C}_{0}$ of the identity element of $\operatorname{SL}(2, \mathbb{C})$, as we would expect if we naively set $\xi_{0}=0$ in expression (2.13) for $U$.

In fact, the conjugacy class $\mathfrak{C}_{\xi}$ can be defined by the equation

$$
\operatorname{Tr} U=\exp \left(-2 \pi \xi_{0}\right)+\exp \left(2 \pi \xi_{0}\right) .
$$

The limit of this equation for $\xi_{0}=0$ is

$$
\operatorname{Tr} U=2,
$$

which is obeyed by $U^{\prime}$. In fact, the equation $\operatorname{Tr} U=2$ defines a union of two conjugacy classes: one conjugacy class $\mathfrak{C}^{\prime}$ that contains $U^{\prime}$, and a second class $\mathfrak{C}_{0}$ that consists of a single element, the identity element of $\mathrm{SL}(2, \mathbb{C})$. 
This gives us a new perspective on why the surface operator defined by generic values of $\alpha, \beta, \gamma$ can have for a limit the surface operator associated with solution (2.5) of Nahm's equations. The former surface operator is associated with monodromy in the class $\mathfrak{C}_{\xi}$. The latter one is associated with monodromy that is generically in the class $\mathfrak{C}^{\prime}$, but can also be in the class $\mathfrak{C}_{0}$, corresponding to trivial monodromy, in the special case $f=0$. The limit of $\mathfrak{C}_{\xi}$ for $\xi \rightarrow 0$ is the union of $\mathfrak{C}^{\prime}$ and $\mathfrak{C}_{0}$. This is why the limit of the generic surface operator can be the one associated with Nahm's equations.

The conjugacy class $\mathfrak{C}^{\prime}$ is not closed in $\operatorname{SL}(2, \mathbb{C})$, because the matrix $U^{\prime}$ of equation (2.11) jumps from being in the class $\mathfrak{C}^{\prime}$ to the class $\mathfrak{C}_{0}$ when $w$ becomes 0 . The closure of $\mathfrak{C}^{\prime}$ therefore includes the point $\mathfrak{C}_{0}$. When we say that the monodromy associated with a given surface operator is in the conjugacy class $\mathfrak{C}^{\prime}$, we will always mean that it is generically in that conjugacy class and in general is in the closure of the stated conjugacy class.

An element of a complex Lie group - $\mathrm{SL}(2, \mathbb{C})$ in our example - is called semisimple if it can be diagonalized (or conjugated to a maximal torus). As in our example, the conjugacy class of a semisimple element is always closed. We call this a semisimple conjugacy class. By contrast, an element $U$ is called unipotent if, in any finite-dimensional representation, it takes the form $U=\exp (n)$, where $n$ is nilpotent. In our above example, $U^{\prime}$ is unipotent. The conjugacy class of a unipotent element is called a unipotent conjugacy class. As in our above example, a unipotent class of positive dimension is never closed; its closure always contains the class $\mathfrak{C}_{0}$ of the identity element of $G_{\mathbb{C}}$. In general, for a group of higher rank, the closure of a unipotent conjugacy class is a union of many (but only finitely many) conjugacy classes.

If a surface operator is associated with a semisimple or unipotent conjugacy class, we call it a semisimple or unipotent surface operator.

\subsubsection{Counting dimensions}

In our above example, the conjugacy class $\mathfrak{C}_{0}$ consists of a single point, whereas $\mathfrak{C}^{\prime}$ has complex dimension 2 or real dimension 4 . Let us understand this from the point of view of Hitchin's equations. To get trivial monodromy, we must set $f=0$ in (2.5). This involves adjusting one real parameter. In addition, at $f=0$, the solution reduces to $A=\phi=0$, which is invariant under global $\mathrm{SU}(2)$ gauge rotations. In fixing the gauge invariance, one is then free to make global $\mathrm{SU}(2)$ gauge rotations on the other fields, away from the support of the surface operator. As the real dimension of $\mathrm{SU}(2)$ is 3, the real codimension of the locus (in a family of solutions of Hitchin's 
equations, or a family of fields in the path integral) at which the monodromy is trivial rather than being conjugate to $U^{\prime}$ is $1+3=4$.

Now suppose that we compactify $\mathcal{N}=4$ super Yang-Mills theory from four dimensions to two dimensions on a Riemann surface $C$, the fourmanifold being then $\mathbb{R}^{2} \times C$. It is possible to make a topological twist so that supersymmetry is preserved; Hitchin's equations for the pair $(A, \phi)$ are the condition for unbroken supersymmetry [11]. Let $\mathcal{M}_{\mathrm{H}}$ be the moduli space of solutions of Hitchin's equations. It is a hyper-Kahler manifold. In one complex structure, it parametrizes, up to conjugation, homomorphisms from the fundamental group of $C$ to $G_{\mathbb{C}}$, the complexification of $G$. Concretely, if $C$ has genus $g$, and $V_{i}, W_{j}, i, j=1, \ldots, g$ are the monodromies around a complete set of $A$-cycles and $B$-cycles, then such a flat connection corresponds to a solution of the equation

$$
V_{1} W_{1} V_{1}^{-1} W_{1}^{-1} \cdots V_{g} W_{g} V_{g}^{-1} W_{g}^{-1}=1,
$$

modulo conjugation by an element of $G$. The complex dimension of the solution space is thus $2(g-1) \operatorname{dim} G$. (The coefficient of $\operatorname{dim} G$ is obtained by counting the $2 g$ group elements $V_{i}$ and $W_{j}$, and subtracting 1 for the equation and 1 for dividing by conjugation.)

Now include a surface operator, supported on $D=\mathbb{R}^{2} \times p$ for $p$ a point in $C$. We suppose that the surface operator is associated with a conjugacy class $\mathfrak{C}$, which in our above examples is $\mathfrak{C}_{\xi}$ or $\mathfrak{C}^{\prime}$. Let $n$ be the complex dimension of $\mathfrak{C}$. The equation for the monodromies becomes

$$
V_{1} W_{1} V_{1}^{-1} W_{1}^{-1} \cdots V_{g} W_{g} V_{g}^{-1} W_{g}^{-1}=U,
$$

where $U$ may be any element of the class $\mathfrak{C}$ (or in general of its closure). Since $U$ takes values in an $n$-dimensional space, the dimension of the moduli space becomes $2(g-1) \operatorname{dim} G+n$.

For instance, if $\mathfrak{C}^{\prime}$ is the unipotent conjugacy class described above, then $n=2$ and including the surface operator increases the complex dimension of the moduli space by 2 .

\subsubsection{More general conjugacy classes}

For $G=\mathrm{SU}(2)$, the unipotent surface operator that we have described above is not essentially new, in the sense that it is the limit of a semisimple surface operator with parameters $\alpha, \beta, \gamma$ as the parameters go to zero. However, the same construction can be applied for other groups $G$ and in general does give essentially new surface operators. In fact, the construction that we have 
explained above can be directly adapted to give a surface operator for any unipotent conjugacy class $\mathfrak{C} \subset G_{\mathbb{C}}$.

Unipotent elements $U$ of $G_{\mathbb{C}}$ correspond naturally to nilpotent elements $n$ of the Lie algebra $\mathfrak{g}_{\mathbb{C}}$ of $G_{\mathbb{C}}$, via $U=\exp (n)$. It is convenient to think in terms of the Lie algebra. A natural source of nilpotent elements of $G_{\mathbb{C}}$ comes by picking an embedding of Lie algebras $\rho: \mathfrak{s l}(2, \mathbb{C}) \rightarrow \mathfrak{g}_{\mathbb{C}}$. Then the raising (or lowering) operator for this embedding gives us a nilpotent element $n \in \mathfrak{g}_{\mathbb{C}}$.

Conversely, the Jacobson-Morozov theorem states that every nilpotent element $n \in \mathfrak{g}_{\mathbb{C}}$ is the raising operator for some $\mathfrak{s l}(2, \mathbb{C})$ embedding. In fact, up to conjugacy, every nilpotent element is the raising operator of some unitary embedding

$$
\rho: \mathfrak{s u}(2) \rightarrow \mathfrak{g}
$$

of the real Lie algebra of $\mathrm{SU}(2)$ to that of the compact form of $G$. We pause to explain this theorem for $G=\mathrm{SU}(N)$. (A similar verification can be made for the other classical groups $\mathrm{SO}(N)$ and $\mathrm{Sp}(2 N)$.) Every nilpotent element $n$ of $\mathfrak{s l}(N, \mathbb{C})$ can be put in Jordan canonical form. In this form, $n$ is block diagonal with off-diagonal blocks vanishing, as shown here

$$
\left(\begin{array}{llllll}
* & * & * & 0 & 0 & 0 \\
* & * & * & 0 & 0 & 0 \\
* & * & * & 0 & 0 & 0 \\
0 & 0 & 0 & * & * & 0 \\
0 & 0 & 0 & * & * & 0 \\
0 & 0 & 0 & 0 & 0 & *
\end{array}\right)
$$

In this examples, the blocks have sizes $\lambda_{1}=3, \lambda_{2}=2, \lambda_{3}=1$. Moreover, in Jordan canonical form, each diagonal block is a "principal nilpotent element" with 1's just above the main diagonal and all other matrix elements vanishing:

$$
n=\left(\begin{array}{cccccc}
0 & 1 & 0 & 0 & \cdots & 0 \\
0 & 0 & 1 & 0 & \cdots & 0 \\
& & & \ddots & & \\
0 & 0 & 0 & 0 & \cdots & 1 \\
0 & 0 & 0 & 0 & \cdots & 0
\end{array}\right) .
$$

In general, the sizes of the blocks are $\lambda_{1}, \lambda_{2}, \ldots, \lambda_{k}$, where $\lambda_{1}+\lambda_{2}+\cdots+$ $\lambda_{k}=N$, and we may as well assume $\lambda_{1} \geq \lambda_{2} \geq \lambda_{3} \geq \cdots \geq \lambda_{k}$. On the other hand, up to isomorphism, there is one irreducible representation of $\mathrm{SU}(2)$ for each positive integer dimension. If we choose the $\mathrm{SU}(2)$ embedding that 
corresponds to the decomposition $N=\lambda_{1}+\lambda_{2}+\cdots+\lambda_{k}$, then the raising operator is conjugate to a matrix in Jordan canonical form with blocks of the indicated size.

An important special case is the case that $\rho: \mathfrak{s u}(2) \rightarrow \mathfrak{s u}(N)$ is an irreducible representation. Then its raising operator is simply an $N \times N$ matrix of the form in (2.21), up to conjugacy. Such an element is called a principal nilpotent element of $\mathfrak{s u}(N)$.

Now it is clear how to make a surface operator associated with any unipotent conjugacy class $\mathfrak{C} \subset G_{\mathbb{C}}$. We pick an $\mathrm{SU}(2)$ embedding $\rho: \mathfrak{s} u(2) \rightarrow \mathfrak{g}$, and define the surface operator using equation (2.5), where $t_{1}, t_{2}$, and $t_{3}$ are now the images of the standard $\mathrm{SU}(2)$ generators under the chosen embedding.

The classification of $\mathfrak{s u}(2)$ embeddings in $\mathfrak{s u}(N)$ has a close analog for orthogonal and symplectic groups. We need only to know a few facts. Irreducible representations of $\mathfrak{s u}(2)$ are real or pseudoreal according to whether their dimension is odd or even. (A real representation admits an invariant symmetric bilinear form, and a pseudoreal one admits an invariant antisymmetric bilinear form.) In addition, if $R$ is a real or pseudoreal representation (it admits an invariant quadratic form that is either symmetric or antisymmetric), then the direct sum $R \oplus R$ can be endowed with an invariant quadratic form that is either symmetric or antisymmetric, as one prefers.

A homomorphism $\rho: \mathfrak{s u}(2) \rightarrow \mathfrak{s o}(N)$ is the same as an $N$-dimensional real representation of $\mathfrak{s u}(2)$, or in other words an $N$-dimensional representation that admits an invariant symmetric form. If $\rho$ is given by a decomposition $N=\lambda_{1}+\lambda_{2}+\cdots+\lambda_{k}$, then the condition, in view of the facts cited in the last paragraph, is that the $\lambda_{i}$ each either are odd or occur with even multiplicity.

A homomorphism $\rho: \mathfrak{s u}(2) \rightarrow \mathfrak{s p}(2 N)$ is the same as a $2 N$-dimensional pseudoreal representation of $\mathfrak{s u}(2)$. If $\rho$ is given by a decomposition $N=$ $\lambda_{1}+\lambda_{2}+\cdots+\lambda_{k}$, then the condition is that the $\lambda_{i}$ either are even or occur with even multiplicity.

A decomposition $N=\lambda_{1}+\lambda_{2}+\cdots+\lambda_{k}$ is called a partition of $N$, and the $\lambda_{i}$ are called parts. To summarize the above, for $G$ of type $A, B, C$, or $D$, we have the following classification of nilpotent orbits in terms of partitions (see, e.g., [12], Section 5):

$\left(A_{N}\right)$ : partitions of $N+1, \sum \lambda_{i}=N+1$;

$\left(B_{N}\right)$ : partitions of $2 N+1, \sum \lambda_{i}=2 N+1$, with a constraint that the multiplicity of every even part $\lambda_{i}$ is even; 
$\left(C_{N}\right)$ : partitions of $2 N, \sum \lambda_{i}=2 N$, with a constraint that the multiplicity of every odd part $\lambda_{i}$ is even;

$\left(D_{N}\right)$ : partitions of $2 N, \sum \lambda_{i}=2 N$, with a constraint that the multiplicity of every even part $\lambda_{i}$ is even. (Moreover, though this will not be important in the present paper, partitions with all $\lambda_{i}$ even correspond to two nilpotent orbits.)

In what follows, we denote the nilpotent orbit associated with a partition $\lambda$ by $\mathfrak{c}_{\lambda}$, and the corresponding unipotent conjugacy class by $\mathfrak{C}_{\lambda}$.

\subsection{Searching for rigid surface operators}

For any $G$ and any $\rho: \mathfrak{s u}(2) \rightarrow \mathfrak{g}$, the above construction gives a surface operator. But generically it is not rigid. For example, $G_{\mathbb{C}}=\operatorname{SL}(N, \mathbb{C})$ has no rigid conjugacy classes at all, except the central elements. Surface operators associated with central classes have been considered in [2] and will be described in Section 4.3. They are rigid, but they are not good illustrations of the ideas of the present paper as they are too special. Let us explain why $\mathrm{SL}(N, \mathbb{C})$ has no other rigid conjugacy classes.

We consider first the semisimple case. Consider a semisimple element of $\operatorname{SL}(N, \mathbb{C})$, say $U=\operatorname{diag}\left(u_{1}, u_{2}, \ldots, u_{N}\right)$, with $u_{i} \in \mathbb{C}^{*}$. Now let us try to vary the $u_{i}$ in such a way that the conjugacy class $\mathfrak{C}_{U}$ containing $U$ varies smoothly. In doing this, we must preserve the condition

$$
u_{1} u_{2} \cdots u_{N}=1 \text {, }
$$

so as to remain in $\operatorname{SL}(N, \mathbb{C})$. Also, regardless of whether $u_{i}=u_{j}$ or $u_{i} \neq u_{j}$ for some $i, j$, when we vary the $u_{i}$, we want to preserve these conditions, so that the subgroup of $\operatorname{SL}(N, \mathbb{C})$ that commutes with $U$ does not jump. As long as $U$ is not central, so that the $u_{i}$ are not all equal, these conditions allow us to vary at least one parameter. So semisimple conjugacy classes in $\mathrm{SU}(N)$ are never rigid.

Now let us consider unipotent conjugacy classes. The basic case in a sense is the principal unipotent conjugacy class. This is the class of an element $U=\exp (n)$ (or equally well $U=1+n$ ), where $n$ is a principal nilpotent element of the Lie algebra, of the form in $(2.21)$. For $G_{\mathbb{C}}=\mathrm{SL}(2, \mathbb{C})$, we have seen in detail that this conjugacy class is the limit of a semisimple conjugacy class $\operatorname{Tr} U=\exp \left(-2 \pi \xi_{0}\right)+\exp \left(2 \pi \xi_{0}\right)$ for $\xi_{0} \rightarrow 0$. So this conjugacy class is not rigid. Similarly, for any $N$, a principal nilpotent element (2.21) can be 
deformed to the following family:

$$
\widetilde{n}=\left(\begin{array}{cccccc}
0 & 1 & 0 & 0 & \cdots & 0 \\
0 & 0 & 1 & 0 & \cdots & 0 \\
& & & \ddots & & \\
0 & 0 & 0 & 0 & \cdots & 1 \\
a_{N} & a_{N-1} & a_{N-2} & a_{N-3} & \cdots & 0
\end{array}\right)
$$

(The lower right matrix element of $\widetilde{n}$ is set to zero to ensure that $\operatorname{Tr} \widetilde{n}=0$.) Any element of $\mathfrak{s l}(N, \mathbb{C})$ of this form is regular, meaning that the subgroup of $\mathrm{SL}(N, \mathbb{C})$ that commutes with $\widetilde{n}$ has complex dimension $n-1$ (the dimension of a maximal torus). The coefficients $a_{k}$ can be interpreted as $\operatorname{Tr} \widetilde{n}^{k}, k=$ $2, \ldots, N$, the Casimir invariants of this group. A generic regular conjugacy class in the Lie algebra is specified by giving the values of the Casimir invariants; the regular nilpotent element of equation (2.21) is what we get (generically) if we set the Casimir invariants to zero. The deformation from $U=\exp (n)$ to $\widetilde{U}=\exp (\widetilde{n})$ shows that the conjugacy class of $U$ is not rigid and in fact it can be deformed to a generic regular semisimple conjugacy class. This means that, just as we explained in detail for $\mathrm{SU}(2)$, a surface operator constructed using an irreducible embedding $\rho: \mathfrak{s u}(2) \rightarrow \mathfrak{s u}(N)$ is a limit for $\alpha, \beta, \gamma \rightarrow 0$ of the surface operator constructed with the general ansatz (2.4).

In general, any element of $\mathrm{SL}(N, \mathbb{C})$ can be put in the block-diagonal form

$$
\left(\begin{array}{llllll}
* & * & * & 0 & 0 & 0 \\
* & * & * & 0 & 0 & 0 \\
* & * & * & 0 & 0 & 0 \\
0 & 0 & 0 & * & * & 0 \\
0 & 0 & 0 & * & * & 0 \\
0 & 0 & 0 & 0 & 0 & *
\end{array}\right)
$$

where now each diagonal block, say of size $k \times k$, is the product of a scalar "eigenvalue" $u \in \mathbb{C}^{*}$ and a principal unipotent element of $\mathrm{GL}(k, \mathbb{C})$. Such a conjugacy class is not rigid if $k>1$ (for any block), since then we can make in that block the argument of the last paragraph. If the blocks are all $1 \times 1$ blocks, we are back in the case, treated first, that $U$ is diagonalizable.

To summarize, we have shown that there are no noncentral rigid conjugacy classes in $\operatorname{SL}(N, \mathbb{C})$. To find rigid (non-central) surface operators, we will have to look farther. 


\subsubsection{Some examples}

However, complex semisimple Lie groups other than $\operatorname{SL}(N, \mathbb{C})$ do have rigid surface operators.

Let us first give some simple examples. For $G=\operatorname{Sp}(2 N)$, we consider the $\mathfrak{s u}(2)$ embedding corresponding to the decomposition

$$
2 N=2+1+1+\cdots+1 .
$$

The corresponding partition is $\lambda=[2,1,1, \ldots, 1]$ which we also write as $\lambda=\left[2,1^{2 N-2}\right]$. The Lie algebra of $\operatorname{Sp}(2 N)$ consists of symmetric matrices $n_{i j}$. The raising operator of an $\mathfrak{s u}(2)$ embedding associated to decomposition (2.25) is a rank 1 matrix of the form $n_{i j}=b_{i} b_{j}$, for some vector $b_{i}$. The conjugacy class $\mathfrak{C}_{n}$ of an element $U=\exp (n)$ for such a $n$ is parametrized by $b$ up to $b \rightarrow-b$, and so has complex dimension $2 N$. Indeed, its closure (obtained by allowing $b=0$ ) is simply

$$
\overline{\mathfrak{C}}_{n}=\mathbb{C}^{2 N} / \mathbb{Z}_{2} .
$$

Not coincidentally, this is a hyper-Kahler orbifold. The orbit of any element of a complex semisimple Lie algebra is always hyper-Kahler, as it can be realized as a moduli space of solutions of Nahm's equations [13].

The conjugacy class $\mathfrak{C}_{n}$ is rigid, if $N>1$, simply because it has the smallest dimension of any non-central conjugacy class in $G_{\mathbb{C}}=\operatorname{Sp}(2 N, \mathbb{C})$. To see that $\mathfrak{C}_{n}$ cannot be deformed to a semisimple conjugacy class, note that a non-central semisimple conjugacy class in $\operatorname{Sp}(2 N, \mathbb{C})$ of smallest dimension is the conjugacy class of the element $\operatorname{diag}\left(u, u^{-1}, 1,1, \ldots, 1\right)$. A small calculation shows that the conjugacy class of this element is of complex dimension $2(2 N-1)$, and this exceeds $2 N$ if $N>1$.

For $N=1$, the conjugacy class $\mathfrak{C}_{n}$ is equivalent to the regular unipotent conjugacy class in $\operatorname{SL}(2, \mathbb{C})$ that we analyzed earlier, and is not rigid. This is related to the fact that for $N=1$, the hyper-Kahler orbifold in equation (2.26) can be blown up or deformed (while for $N>1$, this hyper-Kahler singularity has no moduli).

For $G=\mathrm{SO}(N)$, an example of a rigid unipotent conjugacy class can be constructed similarly. The Lie algebra $\mathfrak{s o}(N)$ consists of antisymmetric matrices $a_{i j}$. A minimal (non-zero) nilpotent element of the Lie algebra $\mathfrak{s o}(N)$ corresponds to the decomposition $N=2+2+1+1+\cdots+1$. An element of the Lie algebra corresponding to such a decomposition takes the form $a_{i j}=b_{i} c_{j}-b_{j} c_{i}$, where $b$ and $c$ are vectors obeying $b \cdot b=b \cdot c=c \cdot c=0$ 
(and modulo an action of $\mathrm{SL}(2, \mathbb{C})$ on the pair $b, c)$. The conjugacy class $\mathfrak{C}_{a}$ of $\exp (a)$ has dimension $2 N-6$. For $N>4$, this is the least dimension of any non-central conjugacy class in $\mathrm{SO}(N, \mathbb{C})$, so again this is a rigid conjugacy class.

Rigid unipotent conjugacy classes or rigid nilpotent orbits also exist in exceptional groups (see Appendix A). A (non-central) unipotent conjugacy class of minimal dimension in a complex semisimple Lie group is always rigid, except for $A_{N}$. In the table, we indicate the dimensions of these minimal conjugacy classes.

\begin{tabular}{lccccccccc}
\hline Type & $A_{N}$ & $B_{N}$ & $C_{N}$ & $D_{N}$ & $E_{6}$ & $E_{7}$ & $E_{8}$ & $G_{2}$ & $F_{4}$ \\
\hline $\operatorname{dim}\left(\mathfrak{C}_{\min }\right)$ & $2 N$ & $4 N-4$ & $2 N$ & $4 N-6$ & 22 & 34 & 58 & 6 & 16 \\
\hline
\end{tabular}

\subsubsection{Computing the dimension of a unipotent orbit}

As in the examples just described, it is convenient to be able to compute the dimension of a unipotent conjugacy class in $G_{\mathbb{C}}$, or equivalently of a nilpotent orbit in $\mathfrak{g}_{\mathbb{C}}$. So we pause to explain how to do this.

Let $d$ be the complex dimension of $G_{\mathbb{C}}$, and let $s$ be the complex dimension of the subgroup $G_{\mathbb{C}}^{n} \subset G_{\mathbb{C}}$ of elements that commute with a given $n \in \mathfrak{g}_{\mathbb{C}}$. The dimension of the orbit of $n$ (or of $\exp (n)$ ) is $d-s$. So it suffices to compute $s$.

The element $n$ is the raising operator for some embedding $\rho: \mathfrak{s u}(2) \rightarrow \mathfrak{g}$. We decompose $\mathfrak{g}$ in irreducible representations $\mathcal{R}_{i}$ of $\mathfrak{s u}(2)$ :

$$
\mathfrak{g}=\underset{i=1}{\stackrel{S}{\oplus}} \mathcal{R}_{i}
$$

The subspace of $\mathfrak{g}$ that commutes with the raising operator $n$ is precisely the space of highest weight vectors for the action of $\mathfrak{s u}(2)$. Each irreducible summand $\mathcal{R}_{i}$ has a one-dimensional space of highest weight vectors. So the subspace of $\mathfrak{g}$ that commutes with $n$ is of dimension equal to $s$, the number of summands in (2.27).

For example, one can use this method to compute the dimensions of the minimal unipotent conjugacy classes in $\operatorname{SO}(N, \mathbb{C})$ or $\operatorname{Sp}(2 N, \mathbb{C})$. We leave this to the reader. For another important example, we re-examine the regular unipotent orbit of $\mathrm{SL}(N, \mathbb{C})$. This corresponds to an irreducible $N$-dimensional representation of $\mathfrak{s u}(2)$, and the summands in (2.27) are of dimension $3,5,7, \ldots, 2 N-1$. There are $N-1$ summands. This shows that 
the subgroup of $\mathrm{SL}(N, \mathbb{C})$ that commutes with a principal unipotent element has dimension $N-1$. (Indeed, for $n$ as in (2.21), this subgroup is generated by the matrices $n, n^{2}, \ldots, n^{N-1}$.) The number $N-1$ equals the dimension of the maximal torus, showing that a principal unipotent orbit has the same dimension as a generic semisimple orbit (to which it can be deformed, as we have already discussed).

\subsubsection{Strongly rigid orbits in orthogonal and symplectic groups}

We will now introduce some useful terminology. We will say that an orbit in a Lie algebra (resp. a conjugacy class in a group) is strongly rigid if its dimension is less than the dimension of any nearby orbit (resp. conjugacy class). Strongly rigid orbits are rigid in a very robust way. For suitable $G$, there are also rigid conjugacy classes that are not strongly rigid; this more delicate phenomenon is described momentarily.

A nilpotent element $n \in \mathfrak{g}_{\mathbb{C}}$ is strongly rigid if and only if the corresponding unipotent group element $U=\exp (n)$ is strongly rigid. So as long as we focus on unipotent conjugacy classes, we can equally well work in the group or the Lie algebra.

An equivalent definition is that $U \in G_{\mathbb{C}}$ (or $n \in \mathfrak{g}_{\mathbb{C}}$ ) is strongly rigid if the dimension of its centralizer is greater than the dimension of the centralizer of any nearby element of $G_{\mathbb{C}}$ (or of $\mathfrak{g}_{\mathbb{C}}$ ). In due course, we will also consider a weaker notion that applies to group elements (but not to elements of a Lie algebra): $U \in G_{\mathbb{C}}$ is rigid (but not strongly rigid) if its centralizer includes as a proper subgroup the centralizer of any nearby element of $G_{\mathbb{C}}$. Thus any nearby element has a centralizer that is strictly smaller than that of $U$. (We also use the term weakly rigid to describe an element that is rigid but not strongly rigid.) For unipotent orbits, there is no difference between rigid and strongly rigid.

At the end of Section 2.2, we explained how to classify unipotent orbits in $\mathrm{SO}(N)$ or $\mathrm{Sp}(2 N)$ in terms of partitions. For a unipotent orbit to be strongly rigid, the partition must obey two conditions. We here explain why the conditions are necessary, referring to [12, Section 7.3], for a proof that they are sufficient.

The first condition reflects the fact that the identity orbit of $\mathrm{SO}(2)$ is not rigid. Indeed, $\mathrm{SO}(2)$ is abelian, so every orbit consists of only one point. The identity orbit is rigid in any other orthogonal or symplectic group.

Let us begin with $G=\mathrm{SO}(N)$. Consider a partition $N=\lambda_{1}+\lambda_{2}+\cdots+$ $\lambda_{k}$ in which one of the parts, say $\lambda^{*}$, occurs with multiplicity $r>1$. Let 
$\rho: \mathfrak{s u}(2) \rightarrow \mathfrak{s o}(N)$ be a corresponding homomorphism. The subgroup of $G$ that commutes with $\rho$ and acts only on the summands of dimension $\lambda^{*}$ is $G^{*}=\mathrm{SO}(r)$ if $\lambda^{*}$ is odd, and $G^{*}=\operatorname{Sp}(r)$ if $\lambda^{*}$ is even. (We recall that if $\lambda^{*}$ is even, then $r$ is always also even.) Let $n$ be the raising operator of $\rho$ and $U=\exp (n)$ the corresponding unipotent element. If $\lambda^{*}=2$ and $r=2$, then because of the exceptional property of $\mathrm{SO}(2)$ just noted, we can modify $U$ by multiplying it by an element of $G^{*}$, without changing the dimension of its orbit.

So a partition of $N$ in which an odd part occurs with multiplicity 2 does not lead to a strongly rigid orbit in $\mathrm{SO}(N)$. For example, for $G=\mathrm{SO}(9)$, the orbit labeled by the partition $\lambda=[3,2,2,1,1]$ is not strongly rigid, since the odd number 1 appears with multiplicity 2 . The same reasoning shows that a partition of $2 N$ in which an even part occurs with multiplicity 2 does not lead to a strongly rigid orbit in $\operatorname{Sp}(2 N)$.

Now we consider the second constraint required in order for a unipotent orbit to be rigid. In terms of partitions, this constraint occurs if there are gaps in the sequence of the $\lambda_{i}$. To be precise, arranging the $\lambda_{i}$ so that $\lambda_{1} \geq \lambda_{2} \geq \cdots \geq \lambda_{k}$, the condition is that all positive integers that are less than $\lambda_{1}$ do occur in this sequence with positive multiplicity.

A partition with a gap does not lead to a strongly rigid orbit. We will discuss the case that the gap separates two parts $\lambda_{j}, \lambda_{j+1}$ with $\lambda_{j} \geq \lambda_{j+1}+$ 2. (The other case with a gap is the case that $\lambda_{k} \geq 2$; it can be treated similarly, replacing the numbers $\lambda_{j}$ and $\lambda_{j+1}$ in the following construction with $\lambda_{k}$ and 0.) For odd $\lambda_{j}, \lambda_{j+1}$, a deformation showing that such an orbit is not strongly rigid can be constructed in a subspace involving only the two blocks of size $\lambda_{j}$ and $\lambda_{j+1}$, as shown here for $\lambda_{j}=3, \lambda_{j+1}=1$ :

$$
\left(\begin{array}{llll}
* & * & * & 0 \\
* & * & * & 0 \\
* & * & * & 0 \\
0 & 0 & 0 & *
\end{array}\right)
$$

So we can replace $N$ by $N^{\prime}=\lambda_{j}+\lambda_{j+1}$ and $\mathrm{SO}(N)$ by $\mathrm{SO}\left(N^{\prime}\right)$. (If $\lambda_{j}$ and $\lambda_{j+1}$ are not odd, the corresponding blocks occur with multiplicity at least 2 and we have to keep two blocks of the relevant dimension in making the construction of the next paragraph.)

So we are reduced to the case that $G=\mathrm{SO}(N)$ with a decomposition $N=m+m^{\prime}$, with $m \geq m^{\prime}+2$. We write $U^{\prime}$ for a unipotent element of $\mathrm{SO}(N)$ associated with this embedding. It is the product of principal unipotent elements in the two blocks. (Each is associated with irreducible $\mathfrak{s u}(2)$ 
embedding in that block.) The conjugacy class of $U^{\prime}$ can be deformed to a non-unipotent (but also not semisimple) conjugacy class of the following type. We consider an $\mathrm{SO}(N)$ matrix $U$ that is the direct sum of three blocks: a generic semisimple $2 \times 2$ block, a principal unipotent $(m-2) \times(m-2)$ block, and a principal unipotent $m^{\prime} \times m^{\prime}$ block. Thus $U$ looks something like

$$
U=\left(\begin{array}{cccc}
* & * & 0 & 0 \\
* & * & 0 & 0 \\
0 & 0 & \times & 0 \\
0 & 0 & 0 & \times
\end{array}\right),
$$

where the upper left $2 \times 2$ block is a generic element of $\mathrm{SO}(2)$

$$
\left(\begin{array}{cc}
a & b \\
-b & a
\end{array}\right), a^{2}+b^{2}=1
$$

and the diagonal elements denoted $\times$ in equation (2.29) represent principal unipotent elements of $\mathrm{SO}(m-2)$ and $\mathrm{SO}\left(m^{\prime}\right)$, respectively. A family of matrices conjugate to $U$ for some $a, b$ can as $a \rightarrow 1, b \rightarrow 0$ approach $U^{\prime}$. This is very similar to the relation between $(2.11)$ and $(2.8)$ in the $\operatorname{SL}(2, \mathbb{C})$ example that we studied in detail (and in fact, if we set $m=3, m^{\prime}=1$, and use the fact that $\mathrm{SL}(2, \mathbb{C})$ is a double cover of $\mathrm{SO}(3, \mathbb{C})$, the previous example becomes a special case of the present discussion). The conjugacy class of $U$ has the same dimension as that of $U^{\prime}$, as one can verify by computing the dimension of the subgroups of $\mathrm{SO}(N)$ that commute with $U$ or $U^{\prime}$, using the method $^{2}$ of equation (2.27).

The conditions that we have just described, taken together, completely characterize rigid nilpotent orbits for orthogonal and symplectic gauge groups [12, Section 7.3]. In the following table, we list the rigid nilpotent orbits in classical groups of small rank. In the table, a partition corresponding to a decomposition $N=\lambda_{1}+\lambda_{2}+\cdots+\lambda_{k}$ is denoted simply $\left[\lambda_{1}, \lambda_{2}, \ldots, \lambda_{k}\right]$. In the table, we do not include the orbit of the identity element, though it is rigid for all $G$. (It corresponds to the partition $[1,1, \ldots, 1]$.

\footnotetext{
${ }^{2}$ To be more exact, one can use this method to compute the dimension of the centralizer of $U^{\prime}$ of equivalently the dimension of its conjugacy class. The dimension of the centralizer of $U$ equals the sum of 1 - coming from the fact that $U$ commutes with an $\mathrm{SO}(2)$ that is embedded as the upper left block in $\mathrm{SO}(N)$ - plus the dimension of the conjugacy class of a unipotent element of $\mathrm{SO}(N-2)$ associated with the decomposition $N-2=$ $(m-2)+m^{\prime}$. This dimension can be computed using equation (2.27), and finally one shows that $U$ and $U^{\prime}$ have centralizers of the same dimension.
} 


\begin{tabular}{ccc}
\hline & $\begin{array}{c}\text { Rigid nilpotent } \\
\text { orbit } \mathfrak{c}_{\lambda}\end{array}$ & $\operatorname{dim}\left(\mathfrak{c}_{\lambda}\right)$ \\
\hline$B_{2}$ & {$[2,2,1]$} & 4 \\
$C_{2}$ & {$[2,1,1]$} & 4 \\
$B_{3}$ & {$[2,2,1,1,1]$} & 8 \\
$C_{3}$ & {$[2,1,1,1,1]$} & 6 \\
$B_{4}$ & {$\left[2^{4}, 1\right]$} & 16 \\
& {$\left[2,2,1^{5}\right]$} & 12 \\
$C_{4}$ & {$[2,2,2,1,1]$} & 18 \\
& {$\left[2,1^{6}\right]$} & 8 \\
$D_{4}$ & {$[3,2,2,1]$} & 16 \\
& {$\left[2,2,1^{4}\right]$} & 10 \\
$\ldots$ & $\ldots$ & $\ldots$ \\
\hline
\end{tabular}

\subsection{Strongly rigid semisimple orbits}

For what we have just described, it is equivalent to consider a nilpotent element $n$ of the Lie algebra $\mathfrak{g}_{\mathbb{C}}$ or a unipotent element $U=\exp (n)$ of the group $G_{\mathbb{C}}$. Indeed, $n$ is a strongly rigid element of the Lie algebra if and only if $U$ is a strongly rigid element of the group.

A strongly rigid element $n \in \mathfrak{g}_{\mathbb{C}}$ is always nilpotent, for the following reason. First of all, if $t$ is a non-zero complex number, $n$ and $t n$ always have orbits of the same dimension. On the other hand, if $n$ is not nilpotent, it has non-zero Casimir invariants, which differ from those of $t n$ (if $t$ is close to but not equal to 1 ), showing that $t n$ is not conjugate to $n$. So the orbit of $n$, if $n$ is not nilpotent, can always be deformed to a nearby orbit of the same dimension, namely the orbit of $t n$.

However, it is possible for a semisimple conjugacy class in the group $G$ or $G_{\mathbb{C}}$ (as opposed to an orbit in the Lie algebra) to be strongly rigid. This does not occur for $G=\mathrm{SU}(N)$, as we explained in Section 2.3. But if $G$ is any other simple Lie group, there are strongly rigid semisimple conjugacy classes in $G$. For example, for $G=\mathrm{SO}(N)$, a strongly rigid conjugacy class contains an element of the form

$$
S_{i}=\operatorname{diag}(+1,+1 \ldots,+1, \underbrace{-1,-1, \ldots,-1,-1}_{2 i}),
$$


where the subscript $i$ refers to the total number of pairs of -1 's, and we require $i>1$. The subgroup $G^{S_{i}}$ of $\mathrm{SO}(N)$ that commutes with $S_{i}$ is a double cover of $\mathrm{SO}(N-2 i) \times \mathrm{SO}(2 i)$. The double cover in question might be denoted as $S(O(N-2 i) \times O(2 i))$. Indeed, $S_{i}$ commutes with a block diagonal matrix

$$
\left(\begin{array}{cc}
A & 0 \\
0 & B
\end{array}\right)
$$

where $A \in O(2 i), B \in O(N-2 i)$; and such a matrix is in $\operatorname{SO}(N)$ if $\operatorname{det} A$ $\operatorname{det} B=1$.

For $i>1$, the conjugacy class of $S_{i}$ is strongly rigid, since perturbing the eigenvalues of $S_{i}$ away from \pm 1 causes the dimension of the centralizer to become smaller. After such a perturbation, the orthogonal groups $\mathrm{SO}(N-$ $2 i)$ and $\mathrm{SO}(2 i)$ are replaced by unitary groups or products of unitary and orthogonal groups of lower dimension.

The case $i=1$ is special, because $\mathrm{SO}(2)$ is abelian. We do not change the dimension of the conjugacy class of $S_{1}$ if we deform it so that the lower right $2 \times 2$ block changes from $\operatorname{diag}(-1,-1)$ to a generic element

$$
\left(\begin{array}{cc}
a & b \\
-b & a
\end{array}\right), a^{2}+b^{2}=1
$$

of $\mathrm{SO}(2)$. Hence, the conjugacy class of $S_{1}$ is not strongly rigid. It actually is our first example of a group element that is weakly rigid but not strongly rigid. If we deform the lower right block of $S_{1}$ as in (2.33), its centralizer is reduced from $S(O(N-2) \times O(2))$ to $\mathrm{SO}(N-2) \times \mathrm{SO}(2)$. The centralizer of the nearby conjugacy class is smaller, but has the same dimension. It is of index 2 in the centralizer of $S_{1}$. We discuss this more fully in Section 3.

There is a similar story for $G=\operatorname{Sp}(2 N)$. A rigid element is again conjugate to the element $S_{i}$ of equation (2.31). For $S_{i}$ to be non-central, we need $1 \leq i \leq N-1$. Again, if we deform $S_{i}$ so that its eigenvalues are not \pm 1 , then the dimension of its centralizer becomes less and the dimension of its conjugacy class increases. So these elements are strongly rigid.

It is not hard to show that these are the only rigid semisimple elements in $\mathrm{SO}(N)$ or $\operatorname{Sp}(2 N)$. If $S$ has a pair of eigenvalues $u, u^{-1}$ that do not equal 1 or -1 , then one can vary $u$ without changing the centralizer of $S$. (If there are several eigenvalue pairs all equal to $u, u^{-1}$, then one must vary these pairs while preserving their equality.)

As one can see in the above examples, if $S$ is a rigid semisimple element of $G$, then the subgroup $G^{S}$ of $G$ that commutes with $S$ has the same rank as 

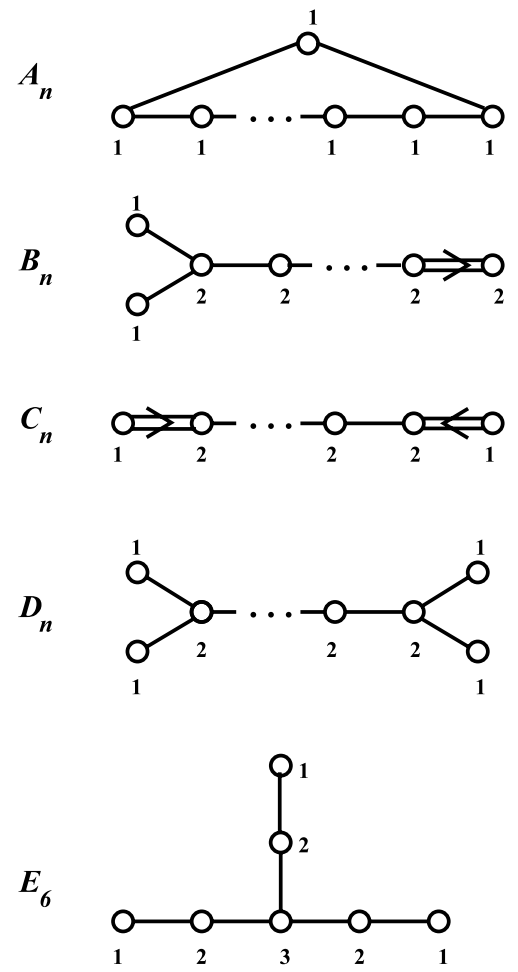
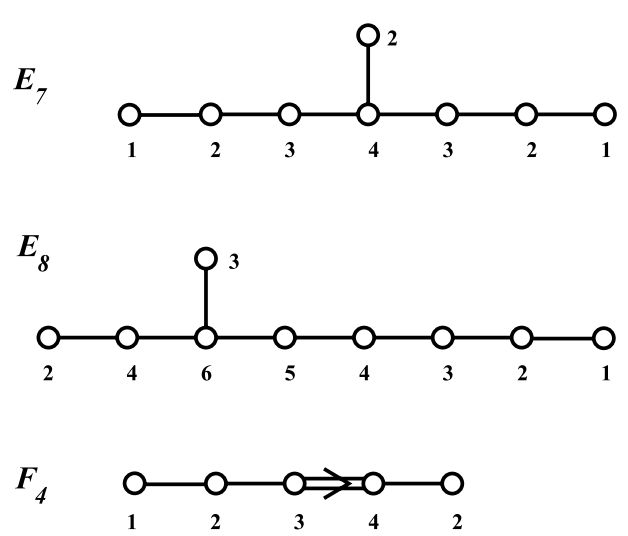

$G_{2}$



Figure 1: Extended Dynkin diagrams for semisimple Lie algebras with Coxeter labels $a_{i}$ (we set $a_{0}=1$ ).

$G$, though of course its dimension is smaller (unless $S$ is central). A further study of the above examples shows that the Dynkin diagram of $G^{S}$ can always be obtained from the extended Dynkin diagram of $G$ by removing one node. (Extended Dynkin diagrams of the simple Lie groups are shown in figure 1.) Finally, the order of $S$ in the adjoint form of $G$ divides the Coxeter label (or Kac number) of the omitted node. For the orthogonal and symplectic groups that are our main examples, this merely means that $S$ is of order 2 , since the relevant labels equal 2 .

In the general theory of rigid semisimple orbits, it is shown that all of these statements hold for any $G$. Indeed, let $\Lambda_{\mathrm{rt}}$ be the root lattice of $G, \Lambda_{\mathrm{rt}}^{+} \subset \Lambda_{\mathrm{rt}}$ the set of positive roots, and $\Delta=\left\{\alpha_{1}, \ldots, \alpha_{r}\right\} \subset \Lambda_{\mathrm{rt}}^{+}$the corresponding set of simple roots. Furthermore, let

$$
\theta=\sum_{i=1}^{r} a_{i} \alpha_{r}
$$


be the highest root in $\Lambda_{\mathrm{rt}}^{+}$. The coefficients $a_{i}$ are the Coxeter labels (or Kac numbers). We denote $\alpha_{0}=-\theta$ and $\bar{\Delta}=\Delta \cup \alpha_{0}$. A proper subset of simple roots, $\Theta \subset \bar{\Delta}$, defines a parabolic subgroup $\mathcal{P}(\Theta) \subset G_{\mathbb{C}}$ with the Levi subgroup $\mathbb{L}(\Theta)$, which will be identified with the centralizer $G^{S}$ of a semisimple element $S$ in $G$. We remind that every parabolic subalgebra $\mathfrak{p}$ has a direct sum decomposition

$$
\mathfrak{p}=\mathfrak{l} \oplus \mathfrak{n}
$$

called Levi decomposition, where $\mathfrak{l}$ is the Levi factor and $\mathfrak{n}$ the nilpotent radical of $\mathfrak{p}$. Specifically, in our case, the parabolic subalgebra $\mathfrak{p}(\Theta)$ associated with the subset of simple roots $\Theta$ is generated by $\mathfrak{t}$ and all the root spaces $\mathfrak{g}_{\alpha}$ such that $\alpha \in \bar{\Delta}$ or $-\alpha \in \Theta$. Similarly, the Levi subalgebra $\mathfrak{l}(\Theta)$ corresponding to $\mathbb{L}(\Theta)$ is

$$
\mathfrak{l}(\Theta)=\mathfrak{t} \oplus \sum_{\alpha \in \Lambda_{\Theta}} \mathfrak{g}_{\alpha}
$$

where $\Lambda_{\Theta}$ denotes the subroot system generated by $\Theta$. We note that, since elements of $\bar{\Delta}$ correspond to nodes of the extended Dynkin diagram of $G$, we can think of $\Theta$ as a subset of nodes of the extended Dynkin diagram.

Since we are interested in rigid surface operators, the centralizer

$$
G^{S}=\mathbb{L}(\Theta)
$$

must be of the same rank as $G$. In other words, $\Theta$ must be a proper subset of $\bar{\Delta}$ obtained by removing a single node; we denote such subsets $\Theta_{i}, i=1, \ldots, r$,

$$
\Theta_{i}=\bar{\Delta} \backslash\left\{\alpha_{i}\right\}
$$

Every such subset of simple roots $\Theta_{i} \subset \bar{\Delta}$, corresponds to (the conjugacy class of) a rigid semisimple element $S_{i}$ in the simply connected form of $G$ (that is $G=G_{\mathrm{sc}}$ ). Generalization to other forms of $G$ (e.g., to the adjoint form $G=G_{\text {ad }}$ ) will be discussed below. [14])

Specifically, we define $S_{0}=1$ and $S_{i}$ for $i=1, \ldots, r$ as follows (see, e.g.,

$$
S_{i}=\exp \left(2 \pi \mathrm{i} \omega_{i}^{\vee} / a_{i}\right)
$$

where $\omega_{i}^{\vee} \in \mathbb{T}$ are the fundamental coweights defined by

$$
\left\langle\alpha_{i}, \omega_{j}^{\vee}\right\rangle=\delta_{i j}
$$

One important consequence of the fact that rigid semisimple elements are of finite order (the order being a divisor of one of the Coxeter labels) is that any rigid semisimple element of $G_{\mathbb{C}}$ can actually be conjugated to the 
compact group $G$. This is important in the context of $\mathcal{N}=4$ super YangMills theory, since only $G$ and not $G_{\mathbb{C}}$ is a group of gauge symmetries in this theory.

We described rigid semisimple elements assuming that $G$ is simply connected. Now we wish to relax this assumption and, in particular, to describe rigid semisimple elements when $G$ is of the adjoint type. Note, that in the construction of rigid semisimple elements in the simply connected form of $G$ we found rigid elements $S_{i}$ for every choice of the proper subset $\Theta_{i} \subset \bar{\Delta}$, where index $i$ runs from 0 to $r$, not taking account symmetries of the Dynkin diagram.

\begin{tabular}{lcccccc}
\hline Type & $A_{N}$ & $B_{N}, C_{N}, E_{7}$ & $D_{2 N}$ & $D_{2 N+1}$ & $E_{6}$ & $E_{8}, F_{4}, G_{2}$ \\
\hline $\mathcal{Z}\left(G_{\text {sc }}\right)$ & $\mathbb{Z}_{N+1}$ & $\mathbb{Z}_{2}$ & $\mathbb{Z}_{2} \times \mathbb{Z}_{2}$ & $\mathbb{Z}_{4}$ & $\mathbb{Z}_{3}$ & 1 \\
\hline
\end{tabular}

The center $\mathcal{Z}\left(G_{\mathrm{sc}}\right)$ of the universal cover $G_{\mathrm{sc}}$ acts on the extended Dynkin diagram, therefore, relating some of the nodes that give rise to the same conjugacy classes of rigid semisimple elements. Hence, if we wish to consider, say, the adjoint form of $G$, we need to divide by the action of $\mathcal{Z}\left(G_{\mathrm{sc}}\right)$ and to take only one conjugacy class for every orbit of the $\mathcal{Z}\left(G_{\mathrm{sc}}\right)$-action on the nodes of the Dynkin diagram. This leads to a similar classification of rigid semisimple elements (and their conjugacy classes) for the adjoint form of $G$, except that now the index $i$ that labels proper subsets $\Theta_{i} \subset \bar{\Delta}$ runs only over the subset of the nodes of the Dynkin diagram not identified by $\mathcal{Z}\left(G_{\mathrm{sc}}\right)$ :

$$
\begin{array}{cl}
\left(A_{N}\right): & i=0, \\
\left(B_{N}\right): & 0 \leq i \leq N-1, \\
\left(C_{N}\right): & 0 \leq i \leq\left[\frac{N}{2}\right], \\
\left(D_{N}\right): & 0 \leq i \leq\left[\frac{N}{2}\right]-1, \\
\left(E_{6}\right): & 0 \leq i \leq 2, \\
\left(E_{7}\right): & 0 \leq i \leq 4, \\
\left(E_{8}, F_{4}, G_{2}\right): & 0 \leq i \leq r .
\end{array}
$$

For example, in type $A$ the center $\mathcal{Z}\left(G_{\text {sc }}\right)$ acts by "rotating" the nodes of the extended Dynkin diagram, in types $B$ and $C$ it acts by "reflection" with respect to the horizontal (resp. vertical) axis, etc. 


\subsubsection{Rigid semisimple surface operators}

Now we will explain why rigid semisimple surface operators are relevant for our purposes.

We will describe a gauge theory singularity in real codimension 2 associated with a rigid semisimple element of $G$. In the notation of Section 2.1, we take the singularity to be at $x^{2}=x^{3}=0$, and we use polar coordinates $x^{2}+\mathrm{i} x^{3}=r \mathrm{e}^{\mathrm{i} \theta}$.

In the absence of any singularity, an adjoint-valued field on the $x^{2}-x^{3}$ plane (for fixed values of the other coordinates $x^{0}, x^{1}$, which we suppress) can be represented by an adjoint-valued function $\Phi(r, \theta)$ that obeys $\Phi(r, \theta+$ $2 \pi)=\Phi(r, \theta)$. If $S$ is any element of the gauge group $G$, we can modify this condition to

$$
\Phi(r, \theta+2 \pi)=S \Phi(r, \theta) S^{-1} .
$$

Since $G$ is a symmetry group of $\mathcal{N}=4$ super Yang-Mills theory, it makes sense to formulate $\mathcal{N}=4$ super Yang-Mills theory for fields that have this sort of behavior, near a codimension two surface $D$ in spacetime.

Of course, if we impose this condition, then along $D$, we should divide only by gauge transformations that commute with $S$. This recipe gives a surface operator that makes sense for any $S \in G$. It varies smoothly as long as the centralizer $G^{S}$ of $S$ in $G$ does not change. To get a rigid surface operator, we must pick $S$ to be rigid, meaning that $G^{S}$ jumps if $S$ is changed at all.

Let us compare the surface operator obtained in this description to the type of surface operator that we considered in [2]. There, as in equation (2.4), we considered a gauge singularity of the form $A=\alpha d \theta$. (For the present purposes, we set $\beta=\gamma=\eta=0$.) One quantizes $\mathcal{N}=4$ super Yang-Mills theory for fields with this type of singularity, dividing by gauge transformations that at $z=0$ are valued in $G^{\alpha}$, the centralizer of $\alpha$ in $G$. Let us call this type of surface operator a generic one.

A generic surface operator behaves well as $\alpha$ is varied as long as the centralizer of $\alpha$ is the same as the centralizer of the monodromy $S=\exp (-2 \pi \alpha)$. We are precisely in the situation in which this is not the case, for if $S$ is strongly rigid (and non-central) then the centralizer of $S$ is strictly larger than the centralizer of any $\alpha \in \mathfrak{g}$ such that $S=\exp (-2 \pi \alpha)$. (Likewise, if $S$ is rigid, then invariance under $G^{S}$ does not allow the introduction of any continuous parameters analogous to $\beta, \gamma, \eta$.) 
The rigid surface operator with monodromy a rigid semisimple element $S \in G$ is therefore not quite a special case of the generic construction in [2]. But it is a close cousin, somewhat similar to the construction in [2] of surface operators associated with Levi subgroups $\mathbb{L}$ that are strictly larger than $\mathbb{T}$.

\subsection{Combining the two constructions}

So far we have constructed rigid surface operators whose monodromy is a rigid element of $G_{\mathbb{C}}$ that is either unipotent or semisimple. The former construction used Nahm's equations and the latter one was done by fiat in equation (2.41). Actually, we can combine the two constructions and construct a rigid surface operator whose monodromy is in any rigid conjugacy class of $G_{\mathbb{C}}$, not necessarily semisimple or unipotent.

We need to know a few facts. To being with, any element $V \in G_{\mathbb{C}}$ can be written as $V=S U$, where $S$ is semisimple, $U$ is unipotent, and $S$ commutes with $U$. Moreover, let $G_{\mathbb{C}}^{S}$ be the centralizer of $S$ in $G_{\mathbb{C}}$, so $U \in G_{\mathbb{C}}^{S}$. Then the condition for $V=S U$ to be rigid (or strongly rigid) in $G_{\mathbb{C}}$ is that $S$ must be rigid (or strongly rigid) in $G_{\mathbb{C}}$ and $U$ must be rigid in $G_{\mathbb{C}}^{S}$.

To construct a surface operator with monodromy $V=S U$, we combine the two constructions as follows. First we require that near $r=0$, all fields of $\mathcal{N}=4$ super Yang-Mills theory obey $\Phi(r, \theta+2 \pi)=S \Phi(r, \theta) S^{-1}$, as in (2.41). Second, we also pick a homomorphism $\rho: \mathfrak{s u}(2) \rightarrow \mathfrak{g}^{S}$ (here $\mathfrak{g}^{S}$ is the Lie algebra of $G^{S}$ ) and we require that the fields have a singularity near $r=0$ that is given by the familiar solution (2.6) of Nahm's equations:

$$
\begin{aligned}
& A=\frac{t_{1} d \theta}{\ln r}+\cdots, \\
& \phi=\frac{t_{2} d r}{r \ln r}-\frac{t_{3} d \theta}{\ln r}+\cdots,
\end{aligned}
$$

where the ellipses denote terms that are less singular at $r=0$. Because $\rho$ commutes with $S$, this condition on the fields is compatible with (2.41). The combined condition defines a surface operator with the monodromy

$$
V=S U
$$

There is no need here for $V$ to be rigid. For every conjugacy class in $G_{\mathbb{C}}$, a construction along these lines gives a surface operator of monodromy $V$. However, for generic $V$, this surface operator is simply equivalent to a special case of the generic surface operators constructed in [2] and reviewed 
in Section 2.1. For certain well-chosen $V$, the construction gives something new. The case that is most novel, or at least most different from what was already considered in [2], is the case that $V$ is rigid.

As we explained in the previous subsection, strongly rigid semisimple elements correspond to proper subsets of simple roots. For every such subset $\Theta_{i} \subset \bar{\Delta}$, the corresponding Levi subgroup $\mathbb{L}\left(\Theta_{i}\right)$ is precisely the centralizer $G^{S_{i}}$ of the semisimple element $S_{i}$. In the case of orthogonal and symplectic groups, the Levi subgroup $\mathbb{L}\left(\Theta_{i}\right)$ is always a product of two factors

$$
\mathbb{L}\left(\Theta_{i}\right)=\mathbb{L}^{\prime} \times \mathbb{L}^{\prime \prime},
$$

where to avoid having to specify exceptions we allow the case that $\mathbb{L}^{\prime}$ or $\mathbb{L}^{\prime \prime}$ is trivial and the other is equal to $G$. (This happens if $\Theta_{0}$ is obtained by omitting the extended root, leaving the original Dynkin diagram of $G$. Thus $\Theta_{0}=\Delta$ and $\mathbb{L}\left(\Theta_{0}\right)=G$. We think of the Dynkin diagram of $G$ as the union of itself with an empty Dynkin diagram. We simply include this case in our notation as the case that the product of groups is $\mathbb{L}\left(\Theta_{0}\right)=1 \times G$. This is precisely the case of a unipotent conjugacy class.) We denote by $\mathfrak{l}\left(\Theta_{i}\right)=\mathfrak{l}^{\prime} \oplus \mathfrak{l}^{\prime \prime}$ the Lie algebra of $\mathbb{L}\left(\Theta_{i}\right)$.

After picking $S$, the construction of strongly rigid surface operators with monodromy $V=S U$ also requires a choice of a rigid unipotent $U \in G_{\mathbb{C}}^{S_{i}}$ or, in view of equation (2.44), a pair of rigid nilpotent orbits $\mathfrak{c}^{\prime}$ and $\mathfrak{c}^{\prime \prime}$ in $\mathfrak{l}_{\mathbb{C}}^{\prime}$ and $\mathfrak{l}_{\mathbb{C}}^{\prime \prime}$, respectively. A complete classification of rigid nilpotent orbits for classical groups was described in Section 2.2. For such groups, nilpotent orbits are labeled by partitions. Therefore, in such cases we can use a pair of partitions $\left(\lambda^{\prime}, \lambda^{\prime \prime}\right)$ to label nilpotent orbits in $\mathfrak{l}_{\mathbb{C}}^{\prime} \oplus \mathfrak{l}_{\mathbb{C}}^{\prime \prime}$. To summarize, strongly rigid conjugacy classes in $G_{\mathbb{C}}$ are labeled by the choice of a root system $\Theta_{i} \subset \bar{\Delta}$ and a rigid nilpotent orbit in each factor of $\mathfrak{l}\left(\Theta_{i}\right)$; in classical types $A, B, C$, and $D$ we can naturally label such rigid conjugacy classes by a pair of partitions,

$$
\mathfrak{C}_{\left(\lambda^{\prime}, \lambda^{\prime \prime}\right)}^{\Theta_{i}} \subset G_{\mathbb{C}}
$$

For instance, in Section 5 we consider many examples of dual pairs of rigid surface operators in theories with gauge groups $G=\operatorname{Sp}(2 N)$ and ${ }^{L} G=$ $\mathrm{SO}(2 N+1)$. Strongly rigid semisimple conjugacy classes in these theories are labeled by a choice of node $i=0,1, \ldots, N$ that defines the root system $\Theta_{i} \subset \bar{\Delta}$ and a pair of partitions $\left(\lambda^{\prime}, \lambda^{\prime \prime}\right)$. Omitting the $i$ th node from the extended Dynkin diagram of $B_{N}$ gives the root system of type

$$
D_{i} \times B_{N-i}
$$


that we already described explicitly in equation (2.32). Hence, in the case of $B_{N}$ both partitions $\lambda^{\prime}$ and $\lambda^{\prime \prime}$ are orthogonal. Similarly, omitting the $i$ th node from the extended Dynkin diagram of $C_{N}$ gives the root system of type

$$
C_{i} \times C_{N-i}
$$

and the corresponding partitions $\lambda^{\prime}$ and $\lambda^{\prime \prime}$ are symplectic. One of the factors can be absent, in which case the corresponding partition is empty. This is the case of a rigid unipotent conjugacy class.

\section{Alternative point of view}

We begin this section by proposing an alternative point of view about the surface operators constructed in [2] and reviewed in Section 2. In fact, we will take an "electric" viewpoint in which a surface operator is constructed not by postulating a singularity (the "magnetic" point of view) but by introducing additional variables.

With this as our starting point, we will then describe some constructions of rigid surface operators that are more delicate than those of Section 2. This will also lead to our first duality conjecture.

\subsection{Coupling to sigma models}

We simply couple four-dimensional super Yang-Mills theory to hypermultiplets that are supported on a two-manifold $D$ that is to be the support of our surface operator. The hypermultiplets parametrize a hyper-Kahler manifold $\mathcal{Q}$ with $G$ action, so that the supersymmetric sigma model with target $\mathcal{Q}$ can be coupled to supersymmetric Yang-Mills theory with gauge group $G$. Of course, the gauge theory is defined on all of $\mathbb{R}^{4}$, with coordinates $x^{0}, x^{1}, x^{2}, x^{3}$, while the sigma model is defined on the two-dimensional subspace $x^{2}=x^{3}=0$.

Hitchin's equations

$$
\begin{aligned}
& F_{A}-\phi \wedge \phi=0, \\
& d_{A} \phi=0, \quad d_{A} \star \phi=0
\end{aligned}
$$

assert the vanishing of the moment map for the fields $A, \phi$ (regarded as in Section 2.1 as hypermultiplets in a two-dimensional sense). This being so, it is straightforward to include a $\mathcal{Q}$-valued hypermultiplet supported 
at the origin of the $x^{2}-x^{3}$ plane. Let $\vec{\mu}=\left(\mu_{l}, \mu_{2}, \mu_{3}\right)$ be the hyper-Kahler moment map for the action of $G$ on the hyper-Kahler manifold $\mathcal{Q}$. Then the components of $\vec{\mu}$ appear as delta function contributions in Hitchin's equations, which can be written in the form

$$
\begin{aligned}
F_{z \bar{z}}-[\varphi, \bar{\varphi}] & =2 \pi \delta^{2}(\vec{x}) \mu_{1}, \\
\bar{\partial}_{A} \varphi & =\pi \delta^{2}(\vec{x})\left(\mu_{2}+\mathrm{i} \mu_{3}\right),
\end{aligned}
$$

where $\phi=\varphi d z+\bar{\varphi} d \bar{z}$, and $\delta^{2}(\vec{x})$ is a delta function supported at $z=x^{2}+$ $\mathrm{i} x^{3}=0$; the precise numerical factors multiplying the delta functions on the right-hand side depends on a choice of normalization of the hyper-Kahler metric on $\mathcal{Q}$. The labeling of the components of $\vec{\mu}$ as $\left(\mu_{1}, \mu_{2}, \mu_{3}\right)$ depends on a choice ${ }^{3}$ that is made when the supersymmetric sigma model of target space $\mathcal{Q}$ is coupled to the gauge theory.

To decide whether this construction makes sense, we will explore the solutions of Hitchin's equations with the indicated delta function source terms. If there are no reasonable classical solutions, we surmise that the sigma model with target $\mathcal{Q}$ cannot be coupled to the four-dimensional gauge theory. We will see that for suitable $\mathcal{Q}$, there are reasonable classical solutions.

Since the delta function "source" term in equation (3.2) is rotationinvariant, it is reasonable to look for a rotation-invariant solution. So away from $r=0$, we simply make the familiar rotation-invariant ansatz

$$
\begin{aligned}
& A=a(r) \mathrm{d} \theta+f(r) \frac{\mathrm{d} r}{r}, \\
& \phi=b(r) \frac{\mathrm{d} r}{r}-c(r) \mathrm{d} \theta .
\end{aligned}
$$

And, just as in Section 2.1, this leads to Nahm's equations (2.3):

$$
\begin{aligned}
& \frac{\mathrm{d} a}{\mathrm{~d} s}=[b, c], \\
& \frac{\mathrm{d} b}{\mathrm{~d} s}=[c, a], \\
& \frac{\mathrm{d} c}{\mathrm{~d} s}=[a, b]
\end{aligned}
$$

\footnotetext{
${ }^{3}$ The space of pairs $A\left(x^{2}, x^{3}\right), \phi\left(x^{2}, x^{3}\right)$ is an infinite-dimensional hyper-Kahler manifold $\mathcal{W}$, with three independent complex structures. Likewise, $\mathcal{Q}$ is a finite-dimensional hyper-Kahler manifold. In constructing the coupling of $\mathcal{N}=4$ super Yang-Mills theory to the sigma model with target $\mathcal{Q}$, one can use any hyper-Kahler structure on the product $\mathcal{W} \times \mathcal{Q}$. To endow this product with a hyper-Kahler structure, one needs to pick a way of "aligning" the complex structures on the two factors. A choice of such an alignment is equivalent to a choice of what we mean by the components $\mu_{1}, \mu_{2}, \mu_{3}$ of $\vec{\mu}$.
} 
away from $r=0$. What do the delta functions in equation (3.2) mean? Suppose that $a(r)$ has a limit for $r \rightarrow 0$ and call this limit $\alpha$. Then $A \sim \alpha d \theta$ for $r \rightarrow 0$. A connection of this form is flat away from $r=0$, but has delta function curvature $2 \pi \alpha \delta^{2}(x)$. Similarly, if the functions $b(r)$ or $c(r)$ have non-zero limits for $r \rightarrow 0$, this gives delta function contributions in Hitchin's equations. So it is reasonable to interpret the delta functions source terms in Hitchin's equations to mean that we want a solution of Nahm's equations that has the property that the functions $a, b, c$ have limits for $r \rightarrow 0$, and moreover

$$
\lim _{r \rightarrow 0}(a, b, c)=\left(\mu_{1}, \mu_{2}, \mu_{3}\right) .
$$

This is a very strong condition for the following reason. First of all, $r \rightarrow 0$ corresponds to $s \rightarrow \infty$, so in taking this limit, we need to solve Nahm's equations on an infinite interval. For $a, b, c$ to have limits for $s \rightarrow \infty$, their derivatives with respect to $s$ must vanish in this limit, and then Nahm's equations imply that the limiting values of $a, b, c$ must commute. On the other hand, for a generic $\mathcal{Q}$ and a generic point $p \in \mathcal{Q}$, the components $\mu_{1}, \mu_{2}, \mu_{3}$ are completely generic elements of the Lie algebra $\mathfrak{g}$, and there is no reason at all for them to commute.

We conclude that the coupling of the sigma model with target $\mathcal{Q}$ to the gauge theory only makes sense if there are points in $\mathcal{Q}$ such that the components of $\vec{\mu}$ commute. Moreover, in a sense, these are the only allowed points in the combined system. Actually, this statement will eventually need some refinement.

\subsection{An example}

To test whether this is the right point of view, we would like to give some interesting examples of hyper-Kahler manifolds that according to this criterion can be coupled in the above sense to $\mathcal{N}=4$ super Yang-Mills theory.

The half-BPS surface operators constructed in [2] and reviewed in Section 2.1 depend on the choice of a commuting triple $\alpha, \beta, \gamma \in \mathfrak{t} \subset \mathfrak{g}$. We would like to reinterpret these surface operators as arising from the coupling of $\mathcal{N}=4$ super Yang-Mills theory to a sigma model with some hyper-Kahler target manifold $\mathcal{Q}_{\alpha, \beta, \gamma}$. Such a statement was considered as an approximation in [2], but we will re-interpret it here as an exact statement. (The parameter $\eta$ will then further arise as a theta-like angle in the sigma model with this target, rather as in Section 6 of [2].)

We would like $\mathcal{Q}_{\alpha, \beta, \gamma}$ to have the following two properties: 
(1) For any triple $(a, b, c)=g(\alpha, \beta, \gamma) g^{-1}$ that is conjugate to $(\alpha, \beta, \gamma)$, with $g$ an element of $G$, there is a point in $\mathcal{Q}_{\alpha, \beta, \gamma}$ with $\vec{\mu}=(a, b, c)$.

(2) Conversely, any point $p \in \mathcal{Q}_{\alpha, \beta, \gamma}$ such that the components of $\vec{\mu}(p)$ commute is of this type, for some $g \in G$.

Remarkably, Kronheimer [15] has constructed a family of hyper-Kahler manifolds $\mathcal{Q}_{\alpha, \beta, \gamma}$ with precisely these properties. These manifolds are constructed as solution spaces of Nahm's equations on the half-line $s \geq 0$ for three $\mathfrak{g}$-valued fields $X_{1}, X_{2}, X_{3}$ :

$$
\frac{d X_{i}}{d s}+\left[X_{i+1}, X_{i-1}\right]=0, i=1,2,3 .
$$

The equations are to be solved on the half-line $s \geq 0$ with the condition that for $s \rightarrow \infty, \vec{X}(s)$ has a limit which is conjugate to $(\alpha, \beta, \gamma)$. Moreover, the hyper-Kahler moment map turns out to be

$$
\vec{\mu}=\left(X_{1}(0), X_{2}(0), X_{3}(0)\right) .
$$

Given these facts, it is almost a tautology to see that properties (1) and (2) are satisfied. If $(a, b, c)$ is any commuting triple that is conjugate to $(\alpha, \beta, \gamma)$, then the constant solution of Nahm's equations with $\left(X_{1}(s), X_{2}(s), X_{3}(s)\right)=$ $(a, b, c)$ obeys Kronheimer's boundary conditions and defines a point $p \in$ $\mathcal{Q}_{\alpha, \beta, \gamma}$. In view of $(3.7)$, this point obeys $\vec{\mu}(p)=(a, b, c)$, as required to satisfy condition (1). In condition (2), we are given a point $p$ such that the components of $\vec{\mu}$ commute. According to (3.7), it follows that the initial data $X_{1}(0), X_{2}(0), X_{3}(0)$ in Nahm's equations commute. For such commutative initial data, the solution of Nahm's equations is independent of $s$ (the solution is unique, since the equations are first order, and an $s$-independent set of commuting matrices does obey the equations). The boundary conditions for $s \rightarrow \infty$ are then obeyed only if $X_{1}(0), X_{2}(0), X_{3}(0)$ are conjugate to $\alpha, \beta, \gamma$. This demonstrates that condition (2) is satisfied.

We conclude that the coupling of four-dimensional super Yang-Mills theory to a sigma model with target $\mathcal{Q}_{\alpha, \beta, \gamma}$ gives the same singular behavior for the two-dimensional fields $A, \phi$ as the surface operator constructed in [2] with the same parameters. So we claim that the surface operator can be obtained by coupling the gauge theory to the sigma model. As we have seen, this statement depends crucially on the fact that the coupling of the gauge theory to a hyper-Kahler manifold $\mathcal{Q}$ singles out the "good" points in $\mathcal{Q}$ at which the components of $\vec{\mu}$ commute.

The example that we have just analyzed is related to orbits of semisimple elements in complex Lie algebras. Indeed, Kronheimer shows [15] that, 
in one of its complex structures, $\mathcal{Q}_{\alpha, \beta, \gamma}$ is the orbit in $\mathfrak{g}_{\mathbb{C}}$ of $\xi=\alpha-\mathrm{i} \gamma$, assuming that $\xi$ is regular. (Otherwise, $\mathcal{Q}_{\alpha, \beta, \gamma}$ is in general a blowup of this orbit, depending on $\beta$.) Our next example is similarly related to nilpotent orbits in complex Lie algebras, but we will approach it in a more direct way.

\subsection{Another example}

We take $G=\mathrm{SU}(2)$, and we will consider a very simple example of a hyperKahler manifold with the action of $G$. In fact, we will consider a pair of closely related examples. One example is $Y=\mathbb{R}^{4}$, a flat hyper-Kahler manifold with a natural action of $\mathrm{SU}(2)$. We can think of this example as consisting of a single hypermultiplet ${ }^{4}$ in the representation of $\mathrm{SU}(2)$ that has complex dimension two. The second example is $Y^{\prime}=\mathbb{R}^{4} / \mathbb{Z}_{2}$.

$Y$ is such a simple hyper-Kahler manifold that one might hope that the coupling to $Y$ will make sense if any couplings of $\mathcal{N}=4$ super Yang-Mills to a two-dimensional hypermultiplet make sense. The sigma model with target $Y^{\prime}$ is an orbifold of the sigma model with target $Y$, and so its coupling should make sense if that of the first one does.

$Y=\mathbb{R}^{4}$ is completely rigid as a hyper-Kahler manifold; $\mathbb{R}^{4}$ has no hyperKahler moduli that preserve its flat structure at infinity. So if the coupling to $Y$ makes sense, this really should give us a rigid surface operator.

By contrast, $Y^{\prime}$ has hyper-Kahler moduli - it has a singularity at the origin that can be deformed or resolved. So coupling to $Y^{\prime}$ should not give a rigid surface operator.

It is useful to parametrize $Y$ by four fields $y^{a \dot{a}}, a, \dot{a}=1,2$, where the $\mathrm{SU}(2)$ gauge group acts on the first index and a second $\mathrm{SU}(2)$, which rotates the three complex structures of $Y$, acts on the second. We call the second group $\mathrm{SU}(2)^{\prime}$. The reality condition obeyed by $y^{a \dot{a}}$ is $\bar{y}_{a \dot{a}}=\epsilon_{a b} \epsilon_{\dot{a} \dot{b}} y^{b \dot{b}}$. An expectation value of $y$ breaks $\mathrm{SU}(2) \times \mathrm{SU}(2)^{\prime}$ to a diagonal subgroup that we call $\mathrm{SU}(2)^{\prime \prime}$. The moment map at a given value of $y$ is, of course, $\mathrm{SU}(2)^{\prime \prime}-$ invariant. This ensures that, up to conjugation by the original SU(2) group of gauge transformations, we can write the moment map as

$$
\vec{\mu}=h \vec{t}
$$

where $h=|y|^{2}$ and $\vec{t}$ are the $2 \times 2$ Pauli sigma matrices. (The point is that this formula is invariant under conjugation by an element of $\mathrm{SU}(2)$ together

\footnotetext{
${ }^{4}$ Sometimes this object is called a half-hypermultiplet.
} 
with an $\mathrm{SU}(2)^{\prime}$ rotation acting on the vector $\vec{\mu}$. A possible multiplicative constant in (3.8) has been eliminated by a choice of normalization of the flat hyper-Kahler metric of $Y$.)

The components of $\vec{t}$ do not commute with each other, so if we take literally the idea that we must restrict to points in $Y$ or $Y^{\prime}$ for which the components of $\vec{\mu}$ commute with each other, we must set $h=0$. This implies that $y^{a \dot{a}}=\vec{\mu}=0$, so it means that the solutions of Hitchin's equations have no singularity at $r=0$, and are the same as if there were no surface operator at all.

This conclusion does not seem sensible; it seems that including the hypermultiplet should give a surface operator that is different from the trivial one without the hypermultiplet. What we think is wrong is that although the condition that the components of $\vec{\mu}$ should commute with each other is only satisfied at $y^{a \dot{a}}=0$, this is a singular point on the moduli space (of points at which the components commute) since the components of $\vec{\mu}$ are all quadratic in $y$. Because of this singularity, a proper analysis is more delicate, and we will only give a heuristic argument.

In trying to obey the conditions that $(a, b, c) \rightarrow \vec{\mu}$ for $s \rightarrow \infty$ and that $a, b, c$ should commute with each other, we are driven to take $a, b, c$ to zero for large $s$. If $a, b, c$ had nonzero limits for $s \rightarrow \infty$, we would get a solution of Hitchin's equations with a $1 / r$ singularity at $r=0$. The fact that $a, b, c$ are driven to zero means that instead the singularity is milder than $1 / r$. We have already encountered this situation in Section 2.2 (it is described much more fully in [2, Section 3.3]), and in that context the right answer is the following solution of Nahm's equations in which $a, b, c$ vanish for $s \rightarrow \infty$ :

$$
a=-\frac{t_{1}}{s+1 / f}, \quad b=-\frac{t_{2}}{s+1 / f}, \quad c=-\frac{t_{3}}{s+1 / f} .
$$

We see that $a, b, c$ vanish for $s \rightarrow \infty$, but in fact they are proportional to a multiple of the matrices $\vec{t}$; the multiple vanishes for $s \rightarrow \infty$.

Our proposal is that the coupling to the hypermultiplet $Y$ or $Y^{\prime}$ leads to this type of solution of Hitchin's equation, up to conjugation. Thus, instead of simply claiming that $a, b, c$ vanish at $s \rightarrow \infty$, so as to commute and equal the hyper-Kahler moment map of $Y$ or $Y^{\prime}$, we claim that generically they vanish in this logarithmic fashion, and are proportional to an (asymptotically vanishing) multiple of the matrices $\vec{t}$.

So far it does not matter very much if the hypermultiplet parametrizes $Y$ or $Y^{\prime}$. Now let us consider $Y^{\prime}$ more carefully. In Section 2.2, we analyzed 
a surface operator described by the singularity in (3.9). We showed that its monodromy is generically an element of the regular unipotent conjugacy class $\mathfrak{C}^{\prime}$ of $\operatorname{SL}(2, \mathbb{C})$, and is always an element of the closure $\overline{\mathfrak{C}}^{\prime}$ of this class. As explained in (2.16), $\overline{\mathfrak{C}^{\prime}}$ is defined by the equation $\operatorname{Tr} U=2$, for $U \in$ $\mathrm{SL}(2, \mathbb{C})$. Explicitly, to obey $\operatorname{Tr} U=2$, we write

$$
U=\left(\begin{array}{cc}
1+a & b \\
c & 1-a
\end{array}\right)
$$

and then the condition $\operatorname{det} U=1$ (for $U \in \mathrm{SL}(2, \mathbb{C}))$ gives

$$
a^{2}+b c=0
$$

This is a standard description of the $\mathrm{A}_{1}$ singularity, and defines the complex manifold $\mathbb{C}^{2} / \mathbb{Z}_{2}$. This complex manifold can of course be endowed with an $\mathrm{SU}(2)$-invariant hyper-Kahler structure, whereupon it becomes $Y^{\prime}=\mathbb{R}^{4} / \mathbb{Z}_{2}$.

It is possibly better to carry out this analysis for a nilpotent vector $n \in$ $\mathfrak{s l}(2)$, rather than a unipotent element $U \in \mathrm{SL}(2, \mathbb{C})$. If we define $n$ to be a traceless $2 \times 2$ matrix

$$
n=\left(\begin{array}{cc}
a & b \\
c & -a
\end{array}\right),
$$

then the condition $\operatorname{det} n=0$ (ensuring that $n$ is nilpotent), gives again $a^{2}+$ $b c=0$. Of course, one can map this to the conclusion of the last paragraph by setting $U=\exp (n)=1+n$.

Our proposal for interpreting this result is the following. Let $\mathfrak{C}$ be a unipotent conjugacy class in $G_{\mathbb{C}}$ (equivalently, a nilpotent orbit in $\mathfrak{g}_{\mathbb{C}}$ ) and let $\overline{\mathfrak{C}}$ be its closure. From these data, we can proceed in either of two ways to define a surface operator. To the given unipotent orbit, we can associate an $\mathfrak{s u}(2)$ embedding $\rho: \mathfrak{s u}(2) \rightarrow \mathfrak{g}$, and to this we associate a unipotent surface operator as in Section 2.2. Alternatively, following [13], we can use Nahm's equations to endow $\overline{\mathfrak{C}}$ with a hyper-Kahler structure. Then we can define a surface operator by coupling $\mathcal{N}=4$ super Yang-Mills theory to a sigma model with target $\overline{\mathfrak{C}}$. Our proposal is that the two surface operators made in this way are equivalent.

At least for our example with $\mathrm{SU}(2)$, we can justify this conclusion as follows. For $G=\mathrm{SU}(2)$, the hyper-Kahler manifold $\mathcal{Q}_{\alpha, \beta, \gamma}$ is the EguchiHansen ALE manifold, asymptotic at infinity to $\mathbb{R}^{4} / \mathbb{Z}_{2}$. For $\alpha, \beta, \gamma \rightarrow 0$, one gets the blowdown of the Eguchi-Hansen manifold, namely $Y^{\prime}=\mathbb{R}^{4} / \mathbb{Z}_{2}$. We have already proposed that coupling to the sigma model of $\mathcal{Q}_{\alpha, \beta, \gamma}$ introduces the surface operator characterized by given $\alpha, \beta, \gamma$. So coupling to the sigma 
model of $Y^{\prime}$ should give the limit for $\alpha, \beta, \gamma \rightarrow 0$ of the surface operator of generic $\alpha, \beta, \gamma$. As we explained in Section 2.2, this limit is the surface operator associated to the regular unipotent conjugacy class.

\subsubsection{Cover of a unipotent orbit}

The surface operator associated with $Y^{\prime}$ is not rigid because $Y^{\prime}$ has hyperKahler moduli. By the same token, the surface operator associated to $Y=$ $\mathbb{R}^{4}$ should be rigid, since $Y$ has no moduli.

We can formulate what is happening as follows. $Y^{\prime}$ is associated to the regular unipotent conjugacy class $\mathfrak{C}^{\prime}$, which topologically is $\mathbb{R}^{4} / \mathbb{Z}_{2}$ with the origin omitted. (The origin corresponds to $U=1$, or $n=0$.) So $\mathfrak{C}^{\prime}$ has fundamental group $\mathbb{Z}_{2}$. And $Y^{\prime}$ has the same fundamental group in the orbifold sense. As a result, it is possible to take a double cover of $\mathfrak{C}^{\prime}$ or (after taking the closure) $Y^{\prime}$, giving us $Y=\mathbb{R}^{4}$.

$Y^{\prime}$ can also be deformed to regular semisimple conjugacy classes in $\mathfrak{g}_{\mathbb{C}}$, and these are simply connected. For $G=\mathrm{SU}(2)$, we can be very explicit about this. Going back to (3.12), if we deform the nilpotent $\operatorname{orbit} \operatorname{det} n=0$ to a semisimple orbit $\operatorname{det} n=\mu$, we get the equation $a^{2}+b c=\mu$, which defines a smooth and simply-connected manifold. That manifold is a deformation of the $\mathrm{A}_{1}$ singularity; as a hyper-Kahler deformation of $Y^{\prime}$, it is the EguchiHansen ALE hyper-Kahler manifold. More generally, a regular semisimple conjugacy class in $G_{\mathbb{C}}$ is simply connected for any $G$. So although $\mathfrak{C}^{\prime}$ can be deformed to neighboring conjugacy classes and therefore is not rigid, the fact that $\mathfrak{C}^{\prime}$ has a fundamental group that the neighboring conjugacy classes lack means that $\mathfrak{C}^{\prime}$ has a cover that actually is rigid.

From our viewpoint of Section 2, with surface operators constructed via singularities, it is not immediately apparent that covers of conjugacy classes give new surface operators. From our present viewpoint in which unipotent surface operators are derived by coupling to sigma models, this does seem obvious.

\subsubsection{Semisimple surface operators}

However, this reasoning does not apply directly to semisimple surface operators (or any surface operators whose monodromy is not unipotent). The reason for this is simply that the conjugacy class $\mathfrak{C}_{S}$ of a semisimple element of $G_{\mathbb{C}}$ is typically not hyper-Kahler, or even complex symplectic. ${ }^{5}$ So the

\footnotetext{
${ }^{5}$ This can be illustrated by considering the rigid conjugacy classes that were important in Section 2.4. For example, in $\mathrm{SO}(2 n+1, \mathbb{C})$, consider the orbit of the element $S=$
} 
semisimple surface operators of Section 2.4 cannot be constructed by coupling the four-dimensional gauge theory to a sigma model. Nevertheless, it is possible to construct surface operators associated with covers of orbits. We explain an example in Section 3.4.

Although rigid semisimple conjugacy classes are typically not hyper-Kahler, surface operators associated with them rather magically preserve all supersymmetry and therefore preserve the hyper-Kahler nature of the moduli space of solutions of Hitchin's equations.

\subsection{Rigid surface operator for the dual group}

Coupling to the sigma model with target space $Y=\mathbb{R}^{4}$ gives an example of a rigid surface operator for $G=\mathrm{SU}(2)$. It is the only one we know of if one requires some minimality (see Section 3.5). Interestingly, this surface operator does not make sense for the dual group ${ }^{L} G=\mathrm{SO}(3)$, since the center of $\mathrm{SU}(2)$ acts nontrivially on $Y$, as a result of which $Y$ cannot be regarded as a space with $\mathrm{SO}(3)$ action.

Therefore, we must find a rigid surface operator for ${ }^{L} G=\mathrm{SO}(3)$ that has no simple counterpart for $\mathrm{SU}(2)$. Happily, we can find one by thinking carefully about an example that arose in Section 2.4. Let $S$ be the element of $\mathrm{SO}(3)$

$$
S=\left(\begin{array}{ccc}
1 & 0 & 0 \\
0 & -1 & 0 \\
0 & 0 & -1
\end{array}\right)
$$

The centralizer of $S$ is the group $O(2)$, generated by an $\mathrm{SO}(2)$ subgroup, whose typical element is

$$
\widetilde{S}=\left(\begin{array}{ccc}
1 & 0 & 0 \\
0 & a & b \\
0 & -b & a
\end{array}\right), \quad a^{2}+b^{2}=1
$$

together with

$$
R=\left(\begin{array}{ccc}
-1 & 0 & 0 \\
0 & -1 & 0 \\
0 & 0 & 1
\end{array}\right)
$$


respect to a unit vector $b$, which is uniquely determined up to sign; the space $\mathfrak{C}$ of such $b$ 's is a complexification of $\mathbb{R} \mathbb{P}^{2 n}$, equivalent topologically to $T^{*} \mathbb{R} \mathbb{P}^{2 n}$. This space does not admit an $\mathrm{SO}(2 n+1, \mathbb{C})$-invariant complex symplectic structure, as one can show by considering the stabilizer of a point in $\mathfrak{C}$ and its action on the tangent space. So there is certainly no $\mathrm{SO}(2 n+1, \mathbb{C})$-invariant hyper-Kahler structure. 
The orbit of $S$ is not strongly rigid, since $S$ can be deformed to a nearby element, namely $\widetilde{S}$ (with $b$ and $a-1$ small) whose centralizer has the same dimension. However, the centralizer of $\widetilde{S}$ is a proper subgroup of that of $S$. The centralizer of $\widetilde{S}$ is $\mathrm{SO}(2)$, since $\widetilde{S}$ does not commute with $R$, while the centralizer of $S$ is $O(2)$.

The result of this is that the orbit of $S$ is rigid in a weaker sense. It cannot be deformed to a nearby orbit, such as the orbit of $\widetilde{S}$. One would have to replace the orbit of $S$ by a double cover before it could be so deformed.

Of course, we can describe the orbits explicitly. The orbit of $S$ in the compact group $\mathrm{SO}(3)$ is $\mathrm{SO}(3) / O(2)=\mathbb{R}^{2}$. This has fundamental group $\mathbb{Z}_{2}$ (reflecting the fact that $O(2)$ has two components) and its double cover $\mathbf{S}^{2}=\mathrm{SO}(3) / \mathrm{SO}(2)$ is the orbit of $\widetilde{S}$. The orbits in the complex Lie group $\mathrm{SO}(3, \mathbb{C})$ are topologically the cotangent bundles of $\mathbb{R} \mathbb{P}^{2}$ and $\mathbf{S}^{2}$, respectively.

We can lift $S$ to $\mathrm{SU}(2)$; it becomes

$$
S^{\prime}= \pm\left(\begin{array}{cc}
0 & \mathrm{i} \\
-\mathrm{i} & 0
\end{array}\right)
$$

The centralizer of $S^{\prime}$ is $U(1)$, which is the same as the centralizer of a generic semisimple element of $\operatorname{SL}(2, \mathbb{C})$, so a surface operator with monodromy $S^{\prime}$ is not rigid. That is why this construction gives a rigid surface operator for $\mathrm{SO}(3)$ that has no counterpart for $\mathrm{SU}(2)$.

\subsubsection{Duality conjecture}

We can now state our first duality conjecture. We propose that the two rigid surface operators that we have found are dual to each other. ${ }^{6}$ For SU(2), we have the rigid surface operator associated with $Y$, the double cover of a regular unipotent orbit, and for $\mathrm{SO}(3)$, we have the rigid surface operator associated to the orbit of $S$.

The two surface operators are candidates for being dual to each other because the two orbits are both of complex dimension 2. The significance of this is as follows. When $\mathcal{N}=4$ super Yang-Mills is compactified on a product of Riemann surfaces $\Sigma \times C$, the moduli space of solutions $\mathcal{M}_{\mathrm{H}}(C)$ of solutions of Hitchin's equations on $C$ becomes the target space of an effective sigma model defined on $\Sigma$. As we explained in equation (2.18), including a surface operator associated with an orbit of complex dimension

\footnotetext{
${ }^{6}$ They may be the only non-trivial rigid surface operators for $\mathrm{SU}(2)$ and $\mathrm{SO}(3)$ that are minimal in a certain sense; see Section 3.5.
} 
$n$ (the support of the surface operator being $\Sigma \times p$, for $p$ a point in $C$ ) has the effect of increasing the dimension of $\mathcal{M}_{\mathrm{H}}(C)$ by $n$. In sigma models with hyper-Kahler target space, the dimension of the target space is an invariant under all dualities. (For example, it is proportional to the central charge.) So the number $n$ must be duality-invariant.

The proposed dual pair for $\mathrm{SU}(2)$ and $\mathrm{SO}(3)$ are related in the following elegant way. The rigid surface operator for $\mathrm{SU}(2)$ has been rigidified by taking the double cover of an orbit associated to a non-rigid surface operator. Conversely, for $\mathrm{SO}(3)$ the rigid surface operator can be "derigidified" by taking a double cover, as we explain next.

Taking a cover of a semisimple orbit: A basic idea in Section 3.3 was that it is possible to define a surface operator associated with a cover of a unipotent orbit. Now we would like to explain how to define a surface operator associated to a cover of a semisimple orbit. (We cannot use the same approach as before because semisimple surface operators do not arise by coupling to sigma models.) We illustrate the idea in the context of the orbit of the element $S \in \mathrm{SO}(3)$.

We recall that the basic idea of the construction of a semisimple surface operator with monodromy $S$ is simply that near a two-manifold $D$, all fields have a monodromy

$$
\Phi(r, \theta+2 \pi)=S \Phi(r, \theta) S^{-1}
$$

Now we simply modify the definition by saying that we are given the following additional data along $D$ : a normalized eigenvector $v$ of the monodromy, that is a vector $v$ in the three-dimensional representation of $\mathrm{SO}(3)$ that obeys $S v=v$ and is normalized to $(v, v)=1$. For given $S$, there are two choices of $v$. The two choices are exchanged by the $\mathrm{SO}(3)$ element $R$, so making a choice eliminates the invariance under $R$. This has the effect of replacing the orbit of $S$ with its double cover.

Including the choice of $v$ as part of the definition of a surface operator gives us a new surface operator. In general, this may give new rigid surface operators; we will see examples in Section 5. In the present case, however, taking the double cover gives a non-rigid surface operator. It eliminates the discrete symmetry that prevents us from deforming $S$ to the more generic element $\widetilde{S}$ of equation (3.14). The surface operator associated with the double cover of the orbit of $S$ is the limit as $\widetilde{S} \rightarrow S$ of a surface operator with monodromy $\widetilde{S}$. Equivalently, it can be obtained from a generic surface operator with parameters $\alpha, \beta, \gamma$ by taking $\beta, \gamma \rightarrow 0$ and taking $\alpha$ to a value such that $S$ is equal to the monodromy $\exp (-2 \pi \alpha)$. 
We have explained this procedure in a special case, but the procedure is general. Part of the definition of a semisimple surface operator with monodromy $S$ is that along the support $D$ of the surface operator, the structure group of the gauge group is reduced to $G^{S}$, the centralizer of $S$. The case that the orbit of $S$ is not simply connected is the case that $G^{S}$ is not connected but has several components. In this case, we can define a new surface operator by reducing the structure group along $D$ not to $G^{S}$ but to a subgroup of the same dimension that is a union of some of the components of $G^{S}$. Such subgroups correspond to the possible covers of the orbit of $S$.

\subsection{Minimal surface operators}

Once we construct surface operators as in Section 3.1, by coupling to sigma models defined on a surface, we want to impose some condition of minimality or the problem becomes too open-ended. The reason for this is that there are many hyper-Kahler manifolds $\mathcal{Q}$ with $G$ action. We do not, for example, want to allow $\mathcal{Q} \rightarrow \mathcal{Q} \times \mathcal{Q}^{\prime}$ where $\mathcal{Q}^{\prime}$ is some hyper-Kahler manifold with a trivial action of $G$.

For another example, the hyper-Kahler manifolds $\mathcal{Q}_{\alpha, \beta, \gamma}$ that were described in Section 3.1 deserve to be considered minimal because the locus of "good" points at which the components of $\vec{\mu}$ commute is a homogeneous space for the compact gauge group $G$. This led in our analysis to a surface operator with definite values of the surface operator parameters $\alpha, \beta$, and $\gamma$. If one replaces $\mathcal{Q}_{\alpha, \beta, \gamma}$ with a generic hyper-Kahler manifold with $G$ action, the locus of "good" points will not be a homogeneous space for $G$ and $\alpha, \beta, \gamma$ will not have definite values. The effect of this will be somewhat like promoting $\alpha, \beta, \gamma$ from coupling constants to fields. Although this might give an interesting model, it is not what we want to study in the present paper. For studying gauge theory with gauge group $G$, it is reasonable to think that the basic case is the "irreducible surface operator" or "surface eigen-operator" in which $\alpha, \beta, \gamma$ take definite values.

In some sense, we want our surface operators to obey a condition of minimality. We do not exactly know the right technical notion of minimality, but an approximation to it is that a minimal surface operator has the following property. Let $x$ be a point in the support $D$ of a surface operator. Then any chiral operator $\mathcal{O}(x)$ that can be defined at $x$ is the limit of a "bulk" chiral operator $\mathcal{O}\left(x^{\prime}\right)\left(x^{\prime}\right.$ is a point not in $\left.D\right)$ for $x^{\prime} \rightarrow x$. The notion of a "chiral operator" depends on the choice of a subalgebra of the supersymmetry algebra of $\mathcal{N}=4$ super Yang-Mills theory, and the condition makes sense for any choice. 
The aim is to ensure that, for a minimal surface operator associated with a hyper-Kahler manifold $\mathcal{Q}, \mathcal{Q}$ is related to a single orbit of $G$. For example, if $G=\mathrm{SU}(2)$ and $\mathcal{Q}$ is parametrized by a single hypermultiplet in the twodimensional representation, then the associated surface operator is minimal by the above criterion. But if $\mathcal{Q}$ is parametrized by two or more such hypermultiplets, then one can construct ${ }^{7}$ chiral operators supported only on $D$, so the associated surface operator is not minimal by the above criterion.

However, the condition that we have stated may be too strong. It is only intended as an approximation to a good notion of minimality. The criterion that we stated does have the virtue of being duality invariant.

\section{Fingerprints of surface operators}

Our main goal in the rest of this paper is to learn how rigid surface operators transform under $S$-duality of the $\mathcal{N}=4$ super Yang-Mills theory. To this end, in this section we discuss several characteristics of the corresponding conjugacy classes which are expected to be duality invariant.

\subsection{Invariant polynomials}

To start with, we consider the set of gauge-invariant polynomials $P(\varphi(x))$ of the Higgs field $\varphi(x)$. Such polynomials are half-BPS local operators in the $\mathcal{N}=4$ super Yang-Mills theory. If $P$ is homogeneous of degree $d$, the corresponding operator is a superconformal primary of dimension $d$.

Once one picks an invariant quadratic form on the Lie algebra $\mathfrak{g}$ (corresponding physically to a choice of the gauge coupling), there is a natural map from a $G$-invariant polynomial $P: \mathfrak{g} \rightarrow \mathbb{C}$ to an ${ }^{L} G$-invariant polynomial $\widetilde{P}:{ }^{L} \mathfrak{g} \rightarrow \mathbb{C}$. If $G$ is simply laced, the two Lie algebras coincide and this map is the trivial one. To define the map in general, one uses the fact that $G$-invariant polynomials on $\mathfrak{g}$ correspond naturally to Weyl-invariant polynomials on $\mathfrak{t}$, the Lie algebra of a maximal torus $\mathbb{T} \subset G$. But $G$ and ${ }^{L} G$ have the same Weyl group $\mathcal{W}$, and one can define a Weyl-invariant map from $\mathfrak{t}$ to ${ }_{\mathfrak{t}}$, taking a short coroot to a long coroot and a long coroot to a multiple of a short coroot. (For example, see the Appendix to [2] for further detail. The map from $\mathfrak{t}$ to ${ }^{L} \mathfrak{t}$ is unique up to a constant factor that depends on the gauge coupling and is not relevant for what follows.)

\footnotetext{
${ }^{7}$ If one parametrizes the hypermultiplet in one of its complex structures by a pair of chiral superfields $C^{a}, a=1,2$, then given also a second such hypermultiplet $\widetilde{C}^{a}$, one can form the chiral operator $\epsilon_{a b} C^{a} \widetilde{C}^{b}$.
} 
In $\mathcal{N}=4$ super Yang-Mills theory, $S$-duality sends a local operator $P(\varphi)$ to the corresponding local operator $\widetilde{P}(\varphi)$. This can be demonstrated by first showing explicitly that it is true in the abelian case and then by reducing the general case to the abelian case by Higgsing. (For this, one studies the theory on its Higgs or Coulomb branch, in a vacuum in which the gauge group $G$ is spontaneously broken to the abelian subgroup $\mathbb{T}$. Then the appropriate transformation of half-BPS operators in the underlying $G$ theory can be determined by computing what happens in the effective theory with gauge group $\mathbb{T}$.)

\subsubsection{The dimension}

If a surface operator is related to a conjugacy class $\mathfrak{C} \subset G_{\mathbb{C}}$ (as are all surface operators considered in the present paper), then the most basic invariant of this surface operator is the dimension of $\mathfrak{C}$. Indeed, as we explained in Section 3.4 (see also equation (2.18)), in compactification to two dimensions, including a surface operator associated with $\mathfrak{C}$ has the effect of increasing the dimension of the moduli space of solutions of Hitchin's equations by $\operatorname{dim} \mathfrak{C}$. Since the dimension of the target space of a sigma model is a duality invariant, it follows that $\operatorname{dim}(\mathfrak{C})$ is a duality invariant.

For unipotent surface operators constructed via $\mathfrak{s u}(2)$ embeddings $\rho$ : $\mathfrak{s u}(2) \rightarrow \mathfrak{g}$, the dimension of the corresponding nilpotent orbit $\mathfrak{c}$ can be computed as in Section 2.3 by decomposing $\mathfrak{g}$ into irreducible representations of $\mathfrak{s u}(2)$. Then the total number of irreducible summands in decomposition (2.27) gives the dimension $s$ of the centralizer $G_{\mathbb{C}}^{n}$ of a nilpotent element $n \in \mathfrak{c}$, and the dimension of the nilpotent orbit $\mathfrak{c}$ follows from the formula $\operatorname{dim} \mathfrak{c}=\operatorname{dim}\left(G_{\mathbb{C}}\right)-s$. This also gives the dimension of the conjugacy class $\mathfrak{C}$ containing $U=\exp (n)$.

As we explained in Section 2.2, for classical groups of type $A, B, C$, and $D$, homomorphisms $\rho: \mathfrak{s u}(2) \rightarrow \mathfrak{g}$ or, equivalently, nilpotent orbits $\mathfrak{c} \subset \mathfrak{g}_{\mathbb{C}}$ are labeled by partitions $\lambda=\left[\lambda_{1}, \lambda_{2}, \ldots, \lambda_{k}\right]$, where each part $\lambda_{i}$ represents the size of the $i$ th Jordan block. (The general result is summarized at the end of Section 2.2.) For each of these classical groups, it is straightforward to compute the dimension $s$ of the centralizer $G_{\mathbb{C}}^{n}$ of a nilpotent element $n \in \mathfrak{c}$ associated with a given partition, using equation (2.27). One obtains a simple formula for the dimension of the nilpotent orbit $\mathfrak{c}$ in terms of $\lambda_{i}$ see [12, Section 6.1]:

$$
\begin{aligned}
& \left(A_{N}\right): \quad \operatorname{dim}\left(\mathfrak{c}_{\lambda}\right)=(N+1)^{2}-\sum_{i}\left|\left\{j \mid \lambda_{j} \geq i\right\}\right|^{2}, \\
& \left(B_{N}\right): \quad \operatorname{dim}\left(\mathfrak{c}_{\lambda}\right)=2 N^{2}+N-\frac{1}{2} \sum_{i}\left|\left\{j \mid \lambda_{j} \geq i\right\}\right|^{2}+\frac{1}{2} \sum_{i \text { odd }}\left|\left\{j \mid \lambda_{j}=i\right\}\right|,
\end{aligned}
$$




$$
\begin{aligned}
& \left(C_{N}\right): \quad \operatorname{dim}\left(\mathfrak{c}_{\lambda}\right)=2 N^{2}+N-\frac{1}{2} \sum_{i}\left|\left\{j \mid \lambda_{j} \geq i\right\}\right|^{2}-\frac{1}{2} \sum_{i \text { odd }}\left|\left\{j \mid \lambda_{j}=i\right\}\right|, \\
& \left(D_{N}\right): \quad \operatorname{dim}\left(\mathfrak{c}_{\lambda}\right)=2 N^{2}-N-\frac{1}{2} \sum_{i}\left|\left\{j \mid \lambda_{j} \geq i\right\}\right|^{2}+\frac{1}{2} \sum_{i \text { odd }}\left|\left\{j \mid \lambda_{j}=i\right\}\right| .
\end{aligned}
$$

For example, for $G=\mathrm{SU}(2)$ the regular nilpotent orbit discussed in Section 2.2 is an orbit of a nilpotent element $n$ that consists of a single Jordan block of size 2. As we learned there, the closure of the corresponding unipotent conjugacy class is the familiar $\mathbb{Z}_{2}$ orbifold singularity,

$$
\overline{\mathfrak{C}}_{[2]}=\mathbb{C}^{2} / \mathbb{Z}_{2}
$$

It has complex dimension 2, in agreement with (4.1) for $G=A_{1}$ and $\lambda=[2]$. For ${ }^{L} G=\mathrm{SO}(3)$ the same unipotent conjugacy class would be labeled by $\lambda=[3]$, and equation (4.1), now with $G=B_{1}$, also gives $\operatorname{dim} \mathfrak{C}=2$. In symplectic groups, this example is a special case of a hyper-Kahler $\mathbb{Z}_{2}$ orbifold (2.26), the closure of a minimal nilpotent orbit in $C_{N}$ labeled by $\lambda=\left[2,1^{2 N-2}\right]$. As we explained in Section 2.3 , it is strongly rigid for $N>1$, and has complex dimension $2 N$, as is obvious from (2.26). This is in perfect agreement with (4.1), which gives

$$
\operatorname{dim} \mathfrak{C}_{\left[2,1^{2 N-2}\right]}=2 N^{2}+N-\frac{1}{2}(2 N-1)^{2}-\frac{1}{2}-\frac{1}{2}(2 N-2)=2 N .
$$

The dimension of an orbit is an important invariant, but it is not enough, since many rigid surface operators may be associated with orbits of the same dimension. We will next consider a much more refined invariant.

\subsection{Polar polynomials}

In the presence of any of the surface operators described in Sections 2 and 3, the Higgs field has a singularity. For example, in the presence of a unipotent surface operator at $z=0$, we have

$$
\varphi=\frac{n}{z}+\ldots
$$

where $n$ takes values in a prescribed nilpotent orbit $\mathfrak{c}$, and the ellipses refer to regular terms. There is an analogous expression near a semisimple surface operator, as we discuss presently. 
Given such a singularity in $\varphi$, the invariant polynomials $P(\varphi)$ also generally have singularities. Precisely because $n$ is nilpotent, the singular terms in $P(\varphi)$ are not determined ${ }^{8}$ by the singularity in $\varphi$, but rather depend on the regular part of $\varphi$, the part indicated by ellipses in equation (4.2).

The moduli space $\mathcal{M}_{\mathrm{H}}$ of solutions of Hitchin's equations has a Hitchin fibration $\pi: \mathcal{M}_{\mathrm{H}} \rightarrow \boldsymbol{B}$, where $\boldsymbol{B}$ is parametrized by the values of the invariant polynomials $P(\varphi)$. If including a surface operator increases the dimension of $\mathcal{M}_{H}$ by $d=\operatorname{dim} \mathfrak{C}$, then it must increase the dimension of $\boldsymbol{B}$ by $d / 2$. This means that, looking at all possible choices of $P$, there will be precisely $d / 2$ independent complex parameters, characterizing the singularities of the polynomials $P(\varphi)$, that depend on the regular terms not written explicitly in equation (4.2). The pattern of these singularities gives us a rather refined invariant of a surface operator that we will call its "fingerprint."

Let us illustrate this with a simple example. We consider the (non-rigid) surface operator associated with the regular nilpotent orbit of $\mathrm{SL}(2, \mathbb{C})$, that is the orbit $\mathfrak{c}$ of a nilpotent element

$$
n=\left(\begin{array}{ll}
0 & 1 \\
0 & 0
\end{array}\right) .
$$

As explained in [2] and reviewed in Section 2.2, this surface operator can be regarded as a limit of a "generic" surface operator in gauge theory with $G=\mathrm{SU}(2)$ as $\alpha, \beta, \gamma \rightarrow 0$. Supposing that such a surface operator is inserted at $z=0$, the behavior of $\varphi$ near $z=0$ is

$$
\varphi=n / z+m+m^{\prime} z+\cdots,
$$

with $m, m^{\prime} \in \mathfrak{s l}(2)$. For $G=\mathrm{SU}(2)$, the only independent invariant polynomial is

$$
P(\varphi)=\operatorname{Tr} \varphi^{2}=\frac{2 \operatorname{Tr} n m}{z}+\cdots,
$$

where the ellipses denote regular terms. We see that the singularity of $P(\varphi)$ is characterized by exactly one coefficient, in agreement with the fact that $\frac{1}{2} \operatorname{dim} \mathfrak{c}=1$. Moreover, this coefficient depends on the square-integrable part of $\varphi$, which is free to fluctuate, so it is not a parameter of the surface operator; rather, we can regard it as one of the coordinates that parametrizes the base $\boldsymbol{B}$ of the Hitchin fibration, in the presence of a surface operator whose definition depends only on the choice of orbit $\mathfrak{c}^{9}$

\footnotetext{
${ }^{8}$ Any coefficient in $P(\varphi)$ that is determined by the surface operator would be a variable parameter, and a surface operator with such a parameter would not be rigid. See the next footnote for an example.

${ }^{9}$ If we deform this unipotent but non-rigid surface operator to a more general one with $\varphi=\sigma / z+\cdots$, where now $\sigma=(\beta+\mathrm{i} \gamma) / 2$ takes values in a prescribed semisimple
} 
We can extract from equation (4.5)the statement that $\operatorname{Tr} \varphi^{2}$ has a simple pole at $z=0$,

$$
\operatorname{Tr} \varphi^{2} \simeq \frac{p}{z}+\ldots
$$

This statement must be invariant under duality. The assertion that $p$ can be defined as $2 \operatorname{Tr} n m$, with $n$ and $m$ as above, has no reason to be preserved by duality. For $G=\mathrm{SU}(2)$, the "fingerprint" of this particular type of surface operator is simply the statement that the singularity of $\operatorname{Tr} \varphi^{2}$ is a (variable) simple pole.

The regular unipotent conjugacy class $\mathfrak{C}_{\text {reg }}$ of $\operatorname{SL}(2, \mathbb{C})$ is not rigid, however. Closer to the subject of the present paper is the double cover $\widetilde{\mathfrak{C}}_{\text {reg }}$, which, as we explained in Section 3.3, is weakly rigid. Since taking the cover does not change the invariant polynomials, the weakly rigid surface operator associated with $\widetilde{\mathfrak{C}}_{\text {reg }}$ has the same polar polynomials, namely (4.6). In Section 3.4, we proposed that the dual to this weakly rigid surface operator is a surface operator associated with a semisimple conjugacy class of the element (3.13)in ${ }^{L} G=\mathrm{SO}(3)$ :

$$
S=\left(\begin{array}{ccc}
1 & 0 & 0 \\
0 & -1 & 0 \\
0 & 0 & -1
\end{array}\right)
$$

The conjugacy class $\mathfrak{C}_{S}$ is also weakly rigid since nearby conjugacy classes have strictly smaller stabilizers.

As a test of our duality proposal, we will verify that the surface operator associated with the weakly rigid semisimple conjugacy class $\mathfrak{C}_{S}$ leads to the same simple pole in $\operatorname{Tr} \varphi^{2}$. Let us remember the definition of the semisimple surface operator with monodromy $S$. The fields must all have monodromy $S$ around $z=0$. In particular, the expansion of the $\mathfrak{s o}(3)$-valued field $\varphi$ looks like (see Appendix B for the Lie algebra conventions):

$$
\varphi=z^{-1 / 2}\left(\begin{array}{ccc}
0 & a & b \\
-b & 0 & 0 \\
-a & 0 & 0
\end{array}\right)+\left(\begin{array}{ccc}
0 & 0 & 0 \\
0 & c & 0 \\
0 & 0 & -c
\end{array}\right)
$$

where $a, b$, and $c$ are single-valued functions of $z$ (to get the right monodromy) and regular at $z=0$ (so that $\varphi$ is square-integrable). So

$$
\operatorname{Tr} \varphi^{2}=-\frac{4 a b}{z}+\cdots
$$

conjugacy class, we will get $\operatorname{Tr} \varphi^{2}=a / z^{2}+b / z+\cdots$, where now $a=\operatorname{Tr} \sigma^{2}$ is a parameter of the surface operator, and $b$ depends on the regular part of $\varphi$ and is a function on $\boldsymbol{B}$. 
with the same simple pole as before, though of course with a different interpretation of the residue of the pole. This is in perfect agreement with the proposed duality between the weakly rigid conjugacy classes $\widetilde{\mathfrak{C}}_{\text {reg }}$ and $\mathfrak{C}_{S}$.

Before describing any systematic theory, we will consider by hand another, more representative example. This example involves a pair of strongly rigid orbits in $B_{3}$ and $C_{3}$. As we explained in Section 2.5, strongly rigid orbits in classical groups of type $B$ and $C$ can be conveniently labeled by the proper subset of simple roots $\Theta_{i} \subset \bar{\Delta}$ and a pair of partitions $\left(\lambda^{\prime}, \lambda^{\prime \prime}\right)$. In this notation, in type $B_{3}$ there is a strongly rigid semisimple conjugacy class of dimension 6 which corresponds to the root system $\Theta_{3}=D_{3}$ and the partitions $\lambda^{\prime}=\left[1^{6}\right]$ and $\lambda^{\prime \prime}=[1]$,

$$
\mathfrak{C}_{\left(\left[1^{6}\right],[1]\right)}^{D_{3}}
$$

According to the general formula (2.38), in gauge theory it corresponds to a rigid surface operator, with the holonomy of the $G_{\mathbb{C} \text {-valued gauge connection }}$ $\mathcal{A}=A+\mathrm{i} \phi$ conjugate to the rigid semisimple element

$$
S_{3}=\operatorname{diag}(+1,-1,-1,-1,-1,-1,-1)
$$

which breaks the gauge group $G=\mathrm{SO}(7)$ down to a subgroup

$$
O(6) \subset \mathrm{SO}(7),
$$

as in equation (2.46). The conjugacy class of the element $S_{3}$ is thus $\mathrm{SO}(7) /$ $\mathrm{O}(6)$, and so has dimension 6 .

On the other hand, in $C_{3}$ there is also a strongly rigid conjugacy class of dimension 6 , namely the unipotent conjugacy class labeled by the partition $\lambda^{\prime \prime}=[2,1,1,1,1]$. In gauge theory, it corresponds to a singularity of the Higgs field with a single Jordan block of size 2 (corresponding to the part "2" in the partition $\left.\lambda^{\prime \prime}=[2,1,1,1,1]\right)$,

$$
\varphi=\frac{1}{z}\left(\begin{array}{cccccc}
0 & 1 & 0 & 0 & 0 & 0 \\
0 & 0 & 0 & 0 & 0 & 0 \\
0 & 0 & 0 & 0 & 0 & 0 \\
0 & 0 & 0 & 0 & 0 & 0 \\
0 & 0 & 0 & 0 & 0 & 0 \\
0 & 0 & 0 & 0 & 0 & 0
\end{array}\right)+\cdots
$$

In the notation of Section 2.4, this unipotent conjugacy class corresponds to $\Theta_{0}=\Delta$ and similarly to (4.10) can be denoted

$$
\mathfrak{C}_{\left(\emptyset,\left[2,1^{4}\right]\right)}^{C_{3}}
$$


The fact that both conjugacy classes $\mathfrak{C}_{\left(\left[1^{6}\right],[1]\right)}^{D_{3}}$ and $\mathfrak{C}_{\left(\emptyset,\left[2,1^{4}\right]\right)}^{C_{3}}$ are rigid and have the same dimension strongly suggests that they might be related by electric-magnetic duality.

Further evidence for this comes from comparing the fingerprints of these rigid conjugacy classes. In both cases, we find the following behavior of the Higgs field:

$$
\begin{aligned}
\operatorname{Tr} \varphi^{2} & \simeq \frac{p_{2}}{z}+\cdots, \\
\operatorname{Tr} \varphi^{4} & \simeq \frac{p_{4}}{z^{2}}+\frac{p_{1}}{z}+\cdots, \\
\operatorname{Tr} \varphi^{6} & \simeq \frac{p_{6}}{z^{3}}+\frac{p_{3}}{z^{2}}+\frac{p_{5}}{z}+\cdots .
\end{aligned}
$$

Since the orbits in question are six dimensional, the singularity in the invariant polynomials of $\varphi$ should depend on only $3=6 / 2$ coefficients. Accordingly, the six parameters $p_{1}, \ldots, p_{6}$ will obey three relations. It turns out that one gets the same three relations in the two cases ${ }^{10}$ :

$$
\begin{aligned}
p_{4} & =\frac{1}{2}\left(p_{2}\right)^{2}, \\
p_{6} & =\frac{1}{4}\left(p_{2}\right)^{3}, \\
p_{3} & =\frac{3}{4} p_{1} p_{2} .
\end{aligned}
$$

The first two relations are manifestly invariant under a redefinition of the local complex parameter near the surface operator. It is easy to verify that the last relation is also invariant, given the first two. Indeed, for $z=$ $w+\epsilon w^{2}+\cdots$, we have $\frac{1}{z^{k}} \simeq \frac{1}{w^{k}}-\frac{k \epsilon}{w^{k-1}}+\cdots$, so that

$$
\begin{aligned}
& p_{1} \rightarrow p_{1}-2 \epsilon p_{4}=p_{1}-\epsilon\left(p_{2}\right)^{2}, \\
& p_{3} \rightarrow p_{3}-3 \epsilon p_{6}=p_{3}-\frac{3}{4} \epsilon\left(p_{2}\right)^{3} .
\end{aligned}
$$

The first transformation implies $p_{1} p_{2} \rightarrow p_{1} p_{2}-\epsilon\left(p_{2}\right)^{3}$, which together with the second transformation in (4.16) demonstrates that $p_{3}-\frac{3}{4} p_{1} p_{2}$ is indeed an invariant combination.

We will now explain how to find this structure. To compute these polar polynomials in a theory with gauge group $G=\mathrm{SO}(7)$, it is convenient to

\footnotetext{
${ }^{10}$ In making this comparison, one needs a fact explained in Appendix B. The $S$-duality transformation from $\mathrm{SO}(2 N+1)$ gauge theory to $\operatorname{Sp}(2 N)$ gauge theory maps $\operatorname{Tr} \varphi^{2 k}$, with the trace in the $(2 N+1)$-dimensional representation of $\mathfrak{s o}(2 N+1)$, precisely to $\operatorname{Tr} \varphi^{2 k}$ with the trace in the $2 N$-dimensional representation of $\mathfrak{s p}(2 N)$.
} 
pass to a double cover of the $z$-plane (parametrized by a local coordinate $y=z^{1 / 2}$ ) and to describe this surface operator by a singularity with a generic first-order pole consistent with the gauge symmetry breaking (4.11):

$$
\varphi=y^{-1}\left(\begin{array}{ccc}
0 & a & b \\
-b^{t} & 0 & 0 \\
-a^{t} & 0 & 0
\end{array}\right)+\cdots .
$$

Here, $B=(a, b)$ is a generic six-vector (the upper left and lower right blocks are here $1 \times 1$ and $6 \times 6$ matrices). From this starting point, one can compute $\operatorname{Tr} \varphi^{2 k}$ by adding less singular terms with the same structure as in (4.8) (even powers of $y$ for diagonal blocks, odd powers for off-diagonal blocks). On the other hand, in the dual theory with ${ }^{L} G=\operatorname{Sp}(6)$, the polar polynomials (4.14) can be computed directly, by adding a generic regular term to the singularity (4.12) and evaluating $\operatorname{Tr} \varphi^{2 k}$. In either of these two cases, it is immediate to see that $\operatorname{Tr} \varphi^{2 k}$ has a pole of order $k$ at $z=0$, giving the general form in (4.14). It is also easy in each case to verify the first two relations in (4.15); for this it suffices to compute the coefficient of the leading singularity $z^{-k}$ in $\operatorname{Tr} \varphi^{2 k}, i=1,2,3$. It is a little harder to verify the coefficient of $3 / 4$ in the last relation in (4.15). Actually, as we have observed, this coefficient is determined by reparametrization invariance.

\subsubsection{Kazhdan-Lusztig map}

It quickly becomes cumbersome to describe detailed relations among polynomials as in the above example. An alternative point of view is useful. To explain this point of view, we go back to the $\mathrm{SU}(2)$ example of equation (4.4). It is convenient to write

$$
\varphi(z)=\left(\begin{array}{cc}
* & z^{-1}+* \\
m & *
\end{array}\right),
$$

where all we need to know about the matrix elements denoted $*$ is that they are regular at $z=0$. For any non-zero $z, \varphi(z)$ is regular semisimple, and thus can be conjugated to $\mathfrak{t}_{\mathbb{C}}$.

We recall that for $G_{\mathbb{C}}=\mathrm{SL}(2, \mathbb{C}), \mathfrak{t}_{\mathbb{C}}$ is the abelian Lie algebra of traceless diagonal $2 \times 2$ matrices $\operatorname{diag}(\xi,-\xi)$. For $z \neq 0, \varphi(z)$ is conjugate to

$$
\varphi_{D}=\left(\begin{array}{cc} 
\pm \sqrt{m / z} & 0 \\
0 & \mp \sqrt{m / z}
\end{array}\right) .
$$

(This formula is exact if the matrix elements denoted $*$ in (4.18) are set to zero, and in general is valid near $z=0$.) Of course, the diagonalization of $\varphi$ 
is not quite unique - it is only unique up to a Weyl transformation, with the Weyl group $\mathcal{W}$ acting by exchange of the two eigenvalues or equivalently multiplication by -1 . Moreover, we see in equation (4.19) that as $z$ circles around the origin in the punctured complex $z$-plane, $\varphi_{D}$ undergoes a monodromy. Inevitably, the monodromy element is a Weyl transformation, in this case the unique nontrivial element of the Weyl group of $\mathrm{SU}(2)$.

This procedure can be carried out for every surface operator. Regardless of what singularity or monodromy we require $\varphi$ to have at $z=0, \varphi$ is generically regular semisimple for $z \neq 0$. Hence it can be diagonalized for $z \neq 0$, but this diagonalization is only unique up to a Weyl transformation. Mak-


$\varphi_{D}(z)$, whose monodromy around $z=0$ is an element of $\mathcal{W}$. The element $w \in \mathcal{W}$ that arises for a generic choice of $\varphi$ is an invariant of the surface operator.

This invariant is a compact way to describe the "fingerprint" of a surface operator. Let us explain explicitly for $G=S U(N)$ how a conjugacy class in $\mathcal{W}$ determines the fingerprint defined in terms of polar parts of invariant polynomials $P(\varphi)$. The case of any $G$ is similar. Consider first the case that $w$ is a cyclic permutation of the $N$ eigenvalues $\xi_{1}, \ldots, \xi_{N}$ of a $\mathfrak{t}_{\mathbb{C} \text {-valued }}$ function $\varphi_{D}(z)$. Then $w$-invariance and square-integrability of $\varphi$ (which implies that the eigenvalues are less singular than $1 / z$ ) tells us that

$$
\varphi_{D}(z)=\sum_{m=1}^{N-1} c_{m}(z) b^{(m)}(z),
$$

where the functions $c_{m}(z)$ are regular at $z=0$, and the matrices $b^{(m)}$ are

$$
b^{(m)}(z)=z^{-m / N} \operatorname{diag}\left(1, \omega^{k}, \omega^{2 k}, \ldots, \omega^{(N-1) k}\right),
$$

where $\omega=\exp (2 \pi \mathrm{i} / N)$. (For $N=2$, this reduces to our example (4.19) for $G=\mathrm{SU}(2)$. In general, it is invariant under monodromy in $z$ plus cyclic permutation of eigenvalues.) Clearly, given these data, we can compute the behavior of the invariant polynomials $\operatorname{Tr} \varphi^{k}$ for generic functions $c_{m}(z)$ and thus describe the "fingerprint" of the surface operator. The extension to any Weyl conjugacy class is as follows. A general element $w$ of the Weyl group is obtained from a partition $N=\lambda_{1}+\lambda_{2}+\cdots+\lambda_{k}$. One divides the $N$ eigenvalues of $\varphi_{D}$ in $k$ different groups, with $\lambda_{i}$ elements in the $i$ th group; $w$ acts in each group as a cyclic permutation of the $\lambda_{i}$ eigenvalues. Then $\varphi_{D}(z)$ is a direct sum of blocks, the $i$ th block looking just like (4.20), with $N$ replaced by $\lambda_{i}$. Again, this leads to a determination of the "fingerprint" starting from a conjugacy class in $\mathcal{W}$. 
So instead of describing the fingerprint of a surface operator in terms of the polar behavior of functions $P(\varphi)$, and relations among their coefficients, we can summarize this information by specifying a conjugacy class in $\mathcal{W}$.

Since our surface operators are related to conjugacy classes in $G_{\mathbb{C}}$, we really have here a map from conjugacy classes in $G_{\mathbb{C}}$ to conjugacy classes in $\mathcal{W}$. This map is known as the Kazhdan-Lusztig map. It was originally defined [16] as a map that assigns a conjugacy class of $\mathcal{W}$ to a nilpotent orbit in $\mathfrak{g}_{\mathbb{C}}$. (For us, this definition corresponds to the case of a unipotent surface operator.) Later, it was extended by Spaltenstein [17] to a map from nilpotent orbits in parabolic subalgebras to conjugacy classes in $\mathcal{W}$. This more general version is exactly what we need for identifying the fingerprints of a general surface operator with monodromy $V=S U, S$ being semisimple and $U$ unipotent. We will make use of these mathematical results in Section 6 .

\subsection{Center versus topology}

We will describe one other type of invariant of a surface operator. First we recall some basic observations of [2] about center, topology, and surface operators.

Let $\mathcal{Z}$ or $\mathcal{Z}(G)$ be the center of $G$. A very elementary but important example of a surface operator (which moreover is rigid) is obtained by twisting by an element $\mathfrak{z} \in \mathcal{Z}$. We simply make the construction of equation (2.41), but setting $S=\mathfrak{z}$. This gives a strongly rigid surface operator since the conjugacy class of a central element, as it consists only of a single point, has smaller dimension than any noncentral conjugacy class.

Such a surface operator must have a dual, of course. The center of $G$ is the Pontryagin dual ${ }^{11}$ to the fundamental group of ${ }^{L} G$, and vice versa:

$$
\begin{aligned}
\pi_{1}\left({ }^{L} G\right) & \cong \mathcal{Z}(G)^{\vee} \\
\mathcal{Z}\left({ }^{L} G\right) & \cong \pi_{1}(G)^{\vee} .
\end{aligned}
$$

In gauge theory with gauge group ${ }^{L} G$ on a four-manifold $M$, a basic ingredient is an ${ }^{L} G$ bundle ${ }^{L} E \rightarrow M$. It has a characteristic class $\xi \in H^{2}$ $\left(M, \pi_{1}\left({ }^{L} G\right)\right)$. (For example, $\xi$ is the second Stieffel-Whitney class if ${ }^{L} G=$

\footnotetext{
${ }^{11}$ The Pontryagin dual of a finite group $F$ is $F^{\vee}=\operatorname{Hom}(F, U(1))$.
} 
$\mathrm{SO}(3)$.) Now let $D$ be a closed two-manifold in $M$, and

$$
\widehat{f}: \pi_{1}\left({ }^{L} G\right) \rightarrow U(1)
$$

a homomorphism. We denote this homomorphism as $\psi \rightarrow \exp (\mathrm{i}\langle f, \psi\rangle)$, for $\psi \in \pi_{1}\left({ }^{L} G\right)$ and $f$ in a sense the logarithm of $\widehat{f}$. We can modify ${ }^{L} G$ gauge theory by including in the path integral a factor

$$
\Psi_{\widehat{f}}=\exp \left(\mathrm{i}\left\langle f, \int_{D} \xi\right\rangle\right)
$$

This modification gives a surface operator for every choice of the homomorphism $\widehat{f}$ in (4.23). But according to (4.22), the homomorphism $\widehat{f}$ : $\pi_{1}\left({ }^{L} G\right) \rightarrow U(1)$ corresponds naturally to an element of $\mathcal{Z}(G)$. The proposal in [2] is that the surface operator in $G$ gauge theory whose monodromy is a central element $\mathfrak{z}$ is dual to the surface operator in ${ }^{L} G$ gauge theory associated with the corresponding homomorphism $\widehat{f}$.

\subsubsection{Generalization}

So far nothing is new. Now let us repeat this story beginning with a surface operator associated with a conjugacy class $\mathfrak{C}$ in gauge theory with gauge group $G$. Let $V \in \mathfrak{C}$ be the monodromy around the singularity of the complexified gauge field $\mathcal{A}=A+\mathrm{i} \phi$.

Let $\mathfrak{z}$ be an element of $\mathcal{Z}$. Whatever the original surface operator may be, we can, using the reasoning of Section 2, construct a new one with monodromy $\mathfrak{z} V$. Of course, it may happen that $V$ and $\mathfrak{z} V$ are conjugate. In this case, multiplying by $\mathfrak{z}$ gives nothing new. This happens not infrequently if $V$ is semisimple. But if $V$ and $\mathfrak{z} V$ are not conjugate - which is always the case ${ }^{12}$ if $V$ is unipotent and $\mathfrak{z} \neq 1$ - then the twist by $\mathfrak{z}$ gives a new surface operator. If this happens for all nontrivial elements of $\mathcal{Z}$, we say that we can "observe the center of $G$ " for this particular surface operator.

In general, let $\mathcal{Z}^{V}$ or $\mathcal{Z}(G)^{V}$ be the subgroup of $\mathcal{Z}$ defined by saying that $\mathfrak{z} V$ is conjugate to $V$ for $\mathfrak{z} \in \mathcal{Z}^{V}$. Then we can observe the quotient $\mathcal{Z} / \mathcal{Z}^{V}$, in the sense that this quotient group parametrizes new surface operators that we can make by twisting by an element of the center of $G$.

\footnotetext{
${ }^{12}$ Since $\mathfrak{z}$ is central, it acts in any irreducible representation $R$ of $G$ as a multiple of the identity, say $\overline{\mathfrak{z}}$. Pick a representation $R$ for which $\overline{\mathfrak{z}} \neq 1$. If $V$ is unipotent, its only eigenvalue in any representation is 1 (such a $V$ typically is not completely diagonalizable). Likewise, the only eigenvalue of $\mathfrak{z} V$ is $\overline{\mathfrak{z}}$. So $\mathfrak{z} V$ and $V$ are not conjugate.
} 
Of course, there is a dual to this, as follows. Along the support $D$ of a surface operator, say in gauge theory with gauge group ${ }^{L} G$, the structure group of the gauge bundle is reduced from ${ }^{L} G$ to a group $H$ that is a symmetry of the surface operator. ( $H$ is the subgroup of ${ }^{L} G$ that commutes with $V$ and with the relevant $\mathfrak{s u}(2)$ embedding if $V$ is not semisimple.) The inclusion $\iota: H \rightarrow{ }^{L} G$ determines a natural homomorphism $\iota: \pi_{1}(H) \rightarrow$ $\pi_{1}\left({ }^{L} G\right)$ and hence a subgroup $\iota\left(\pi_{1}(H)\right) \subset \pi_{1}\left({ }^{L} G\right)$. When integrated over $D$, the characteristic class $\xi$ of the gauge bundle actually takes values in this subgroup. This reflects the fact that $H$ is the effective gauge group along $D$.

Hence, when we modify the path integral by including the factor $\Psi_{\widehat{f}}=$ $\exp \left(\mathrm{i}\left\langle f, \int_{D} \xi\right\rangle\right)$, so as to build a new surface operator, some choices of $\widehat{f}$ are irrelevant. We are not interested in those $\widehat{f}$ that are trivial when restricted to $\iota\left(\pi_{1}(H)\right)$. So in $\pi_{1}\left({ }^{L} G\right)^{\vee}$, there is an irrelevant subgroup $\widetilde{\pi}_{1}\left({ }^{L} G\right)^{\vee}$, which classifies homomorphisms that are trivial on $\iota\left(\pi_{1}(H)\right)$. We say that we can observe the topology if $\iota\left(\pi_{1}(H)\right)=\pi_{1}\left({ }^{L} G\right)$, so that $\widetilde{\pi}_{1}\left({ }^{L} G\right)^{\vee}$ is trivial and a twist by any factor $\Psi_{\widehat{f}}$ gives a new surface operator.

In general, the surface operators that we can make in ${ }^{L} G$ gauge theory by twisting by some $\widehat{f}$ are classified by $\pi_{1}\left({ }^{L} G\right)^{\vee} / \widetilde{\pi}_{1}\left({ }^{L} G\right)^{\vee}$. By contrast, the possible twists in $G$ gauge theory by an element of the center were classified by $\mathcal{Z}(G) / \mathcal{Z}(G)^{V}$. Since $\pi_{1}\left({ }^{L} G\right)^{\vee}=\mathcal{Z}(G)$ according to (4.22), we see that duality requires

$$
\mathcal{Z}(G)^{V}=\widetilde{\pi}_{1}\left({ }^{L} G\right)^{\vee}
$$

One special case is that if on one side we can observe the full center of $G$ that is if $\mathcal{Z}(G)^{V}$ is trivial - then on the other side we can observe the full topology, meaning that $\iota\left(\pi_{1}(H)\right)=\pi_{1}\left({ }^{L} G\right)$ (so that a homomorphism that annihilates $\iota\left(\pi_{1}(H)\right)$ is trivial). At the other extreme, the center and topology are completely invisible (for the chosen pair of dual surface operators) if $\mathcal{Z}(G)^{V}=\mathcal{Z}(G)$, or dually if $\iota\left(\pi_{1}(H)\right)$ is trivial and all homomorphisms annihilate it.

Let us see how this works out for the (non-central) rigid surface operators for the pair of groups $\mathrm{SO}(3)$ and $\mathrm{SU}(2)$. We recall that for $\mathrm{SU}(2)$, the rigid surface operator in question is associated with the double cover of the nilpotent cone. It has unipotent monodromy and is associated with an irreducible $\mathfrak{s u}(2)$ embedding. The dual rigid surface operator for $\mathrm{SO}(3)$ is associated with the conjugacy class of the element $S=\operatorname{diag}(1,-1,-1)$. The essential case to consider is $G=\mathrm{SU}(2),{ }^{L} G=\mathrm{SO}(3)$. (The opposite case $G=\mathrm{SO}(3),{ }^{L} G=\mathrm{SU}(2)$ is of little interest, as $\mathrm{SO}(3)$ has trivial center and $\mathrm{SU}(2)$ is simply connected.) 
We can analyze this example as follows. Since the monodromy of the $G$ surface operator is unipotent, we can observe the center of $G$; in other words, $\mathcal{Z}(G)^{V}$ is trivial for this surface operator. Dually, the group $H$ is the subgroup $O(2) \subset \mathrm{SO}(3)$. Any loop in $\mathrm{SO}(3)$ can be deformed to a loop in $\mathrm{SO}(2) \subset O(2)$, so $\iota\left(\pi_{1}(H)\right)=\pi_{1}\left({ }^{L} G\right)$; hence we can observe the topology of ${ }^{L} G$.

\section{Duality for strongly rigid surface operators}

Now, we use the invariants described in the previous section in order to identify dual pairs of rigid surface operators in theories with SO and Sp gauge groups, starting with the simplest examples of small rank. To keep things simple, we will analyze only rigid surface operators that are associated to strongly rigid conjugacy classes. We know that these do not give the whole story, even for $\mathrm{SU}(2)$ and $\mathrm{SO}(3)$; in Section 3.4, we described apparently dual rigid surface operators for these groups that are not associated to strongly rigid conjugacy classes.

However, strongly rigid conjugacy classes are relatively easy to analyze, and this analysis will give many examples of what appear to be dual pairs. We begin with rank 2, the first case in which non-trivial strongly rigid conjugacy classes exist.

\subsection{Duality for $G=\operatorname{SO}(5)$ and ${ }^{L} G=\operatorname{Sp}(4)$}

Strongly rigid conjugacy classes give rigid surface operators in either the adjoint or the simply connected form of the group. That being so, the comparison of $B_{2}$ and $C_{2}$ may appear trivial, since these groups are the same. However, as we will see, even in this case, $S$-duality identifies strongly rigid surface operators in a nontrivial way. First, let us describe strongly rigid surface operators in these theories.

In each case, there is only one strongly rigid unipotent surface operator, which in the $\mathrm{SO}(5)$ (resp. $\mathrm{Sp}(4)$ ) theory corresponds to a rigid unipotent conjugacy class $\mathfrak{C}_{\lambda}$ with $\lambda=[2,2,1]$ (resp. $\lambda=[2,1,1]$ ). These rigid unipotent conjugacy classes in $B_{2}$ and $C_{2}$ are both of dimension 4 , so that one might naively expect that duality maps strongly rigid surface operators associated with these unipotent conjugacy classes into each other. However, by studying the polar polynomials, one can show that this is not the case. 
As we explain in Appendix B, the $S$-duality map between $\operatorname{Sp}(2 N)$ gauge theory and $\mathrm{SO}(2 N+1)$ gauge theory maps $\operatorname{Tr} \varphi^{2 k}$, with the trace in the $2 N$-dimensional representation of $\operatorname{Sp}(2 N)$, to $\operatorname{Tr} \varphi^{2 k}$ with the trace in the $(2 N+1)$-dimensional representation of $\mathrm{SO}(2 N+1)$. On the other hand, for the rigid surface operators associated with unipotent conjugacy classes labeled by $\lambda=[2,2,1]$ and $\lambda=[2,1,1]$ we find that, in both cases, the invariant polynomials $\operatorname{Tr} \varphi^{2 k}$ have the same structure of poles

$$
\begin{aligned}
& \operatorname{Tr} \varphi^{2} \simeq \frac{p_{1}}{z}+\cdots, \\
& \operatorname{Tr} \varphi^{4} \simeq \frac{p_{2}}{z^{2}}+\frac{p_{3}}{z},+\cdots
\end{aligned}
$$

but the relations among the coefficients $p_{i}$ are different. Since both conjugacy classes in question are four dimensional, the polar polynomials should contain only $2=4 / 2$ independent coefficients, and there has to be one relation among the three parameters $p_{1}, p_{2}, p_{3}$. For the conjugacy class $\mathfrak{C}_{[2,1,1]}$ in $C_{2}$, the relation is $p_{2}=\frac{1}{2}\left(p_{1}\right)^{2}$. On the other hand, for the conjugacy class $\mathfrak{C}_{[2,2,1]}$ in $B_{2}$, the relation is different: $p_{2}=\frac{1}{4}\left(p_{1}\right)^{2}$.

However, in both $\mathrm{SO}(5)$ and $\mathrm{Sp}(4)$ theories, there is another rigid surface operator, this time with semisimple monodromy. As explained in Section 2.4 , in each case, the monodromy preserves a subgroup of the gauge symmetry group whose Dynkin diagram can be obtained by removing a node from the extended Dynkin diagram of $B_{2}$ or $C_{2}$, respectively. The relevant node is the middle node, $i=1$. (Removing the other node $i=0$ leads back to the rigid unipotent conjugacy class already considered above.) In the notation of Section 2.5, these rigid semisimple conjugacy classes can be denoted by $\mathfrak{C}_{\left(\left[1^{4}\right],[1]\right)}^{D_{2}}$ and $\mathfrak{C}_{\left(\left[1^{2}\right],\left[1^{2}\right]\right)}^{C_{1} \times C_{1}}$ since they preserve gauge symmetry groups $O(4) \subset \mathrm{SO}(5)$ and $\mathrm{Sp}(2) \times \mathrm{Sp}(2) \subset \mathrm{Sp}(4)$, respectively, cf. (2.46) and (2.47). Summarizing, we have the following list of rigid surface operators:

\begin{tabular}{ccc}
\hline$B_{2}$ & $\operatorname{dim}$ & $C_{2}$ \\
\hline $\mathfrak{C}_{\left(\left[1_{1}\right],[1]\right)}^{D_{2}}$ & 4 & $\mathfrak{C}_{(\emptyset,[2,1,1])}^{C_{2}}$ \\
$\mathfrak{C}_{(\emptyset,[2,2,1])}^{B_{2}}$ & 4 & $\mathfrak{C}_{\left(\left[1_{1}^{2}\right],\left[1^{2}\right]\right)}^{C_{1}, C^{2}}$ \\
\hline
\end{tabular}

where we put what we claim to be dual pairs of strongly rigid surface operators on the same line. In particular, a surface operator associated with the rigid semisimple conjugacy class $\mathfrak{C}_{\left(\left[1^{4}\right],[1]\right)}^{D_{2}}$ has the same set of polar polynomials (5.1), with $p_{2}=\frac{1}{2}\left(p_{1}\right)^{2}$, as the surface operator associated with the unipotent conjugacy class $\mathfrak{C}_{(\emptyset,[2,1,1])}^{C_{2}}$ in $C_{2}$. Similarly, surface operators associated with the conjugacy classes $\mathfrak{C}_{(\emptyset,[2,2,1])}^{B_{2}}$ and $\mathfrak{C}_{\left(\left[1^{2}\right],\left[1^{2}\right]\right)}^{C_{1} \times C_{1}}$ have 
the polar polynomials $(5.1)$ with $p_{2}=\frac{1}{4}\left(p_{1}\right)^{2}$, in complete agreement with the duality.

Further evidence for this identification of dual surface operators comes from comparing discrete invariants discussed in Section 4.3. Thus, surface operators associated with the rigid unipotent conjugacy classes $\mathfrak{C}_{(\emptyset,[2,2,1])}^{B_{2}}$ and $\mathfrak{C}_{(\emptyset,[2,1,1])}^{C_{2}}$ can "detect" the center of the gauge group (in the sense that twisting $\mathcal{Z}(G)$ or $\mathcal{Z}\left({ }^{L} G\right)$ gives rise to new surface operators). In fact, as explained in Section 4.3, the group $\mathcal{Z}(G)^{V}$ (resp. $\mathcal{Z}\left({ }^{L} G\right)^{V}$ ) is trivial for any unipotent conjugacy class.

On the other hand, surface operators associated with the rigid unipotent conjugacy classes $\mathfrak{C}_{(\emptyset,[2,2,1])}^{B_{2}}$ and $\mathfrak{C}_{(\emptyset,[2,1,1])}^{C_{2}}$ cannot "detect" topology. Thus, in the adjoint form of $G=\mathrm{SO}(5)$, the symmetry group $H$ of the surface operator associated with the rigid conjugacy classes $\mathfrak{C}_{(\emptyset,[2,2,1])}^{B_{2}}$ is a double cover of $\operatorname{Sp}(2)$. It has a trivial fundamental group, $\pi_{1}(H)=1$, which means that the image of the natural map $\iota: \pi_{1}(H) \rightarrow \pi_{1}\left({ }^{L} G\right)$ is trivial and all homomorphisms (4.23) annihilate $\iota\left(\pi_{1}(H)\right)$. Hence, in the notation of Section 4.3 , we have $\widetilde{\pi}(G)=\pi_{1}(G)$ and we conclude that the strongly rigid surface operator associated with the conjugacy class $\mathfrak{C}_{(\emptyset,[2,2,1])}^{B_{2}}$ cannot "detect" topology. The analysis of topology for the surface operator associated with a rigid unipotent conjugacy class $\mathfrak{C}_{(\emptyset,[2,1,1])}^{C_{2}}$ is essentially identical since the adjoint form of ${ }^{L} G=\mathrm{Sp}(4)$ is isomorphic to $G=\mathrm{SO}(5)$ and the symmetry group $H$ is also the same.

The situation is reversed for surface operators associated with rigid semisimple conjugacy classes $\mathfrak{C}_{\left(\left[1^{4}\right],[1]\right)}^{D_{2}}$ and $\mathfrak{C}_{\left(\left[1^{2}\right],\left[1^{2}\right]\right)}^{C_{1} \times C_{1}}$, which "detect" the fundamental group but not the center of the gauge group.

Let us explain why this is so. We start with the class $\mathfrak{C}_{\left(\left[1^{4}\right],[1]\right)}^{D_{2}}$ in $B_{2}$, which corresponds to the element $S=\operatorname{diag}(1,-1,-1,-1,-1)$ in $\mathrm{SO}(5)$, the adjoint form of $B_{2}$. This element commutes with $\mathrm{SO}(4)$, and the map of fundamental groups from $\mathrm{SO}(4)$ to $\mathrm{SO}(5)$ is surjective, so the surface operator associated with this class detects topology.

Let us explain why this surface operator does not detect the center of the gauge group. Let $\mathfrak{z}$ be the non-trivial element of the center of Spin(5); of course, $\mathfrak{z}$ corresponds to a $2 \pi$ rotation in space. Let $T=\operatorname{diag}(-1,-1,1,1,1)$ $\in \mathrm{SO}(5)$. As elements of $\mathrm{SO}(5), S$ and $T$ commute, but when they are lifted to $\operatorname{Spin}(5)$, we have

$$
T^{-1} S T=\mathfrak{z} S,
$$


showing that $S$ and $\mathfrak{z} S$ are conjugate in $\operatorname{Spin}(5)$. Thus, a surface operator with monodromy conjugate to $S$ does not detect topology.

The story is similar for the conjugacy class $\mathfrak{C}_{\left(\left[1^{2}\right],\left[1^{2}\right]\right)}^{C_{1} \times C_{1}}$ in $C_{2}$. This corresponds to an element of $C_{2}$ that in $2 \times 2$ blocks looks like

$$
S^{\prime}=\left(\begin{array}{cc}
-1 & 0 \\
0 & 1
\end{array}\right)
$$

In the adjoint form of the group, which is $\operatorname{Sp}(4) / \mathbb{Z}_{2}, S^{\prime}$ commutes with $H=$ $(\operatorname{Sp}(2) \times \operatorname{Sp}(2)) / \mathbb{Z}_{2}$. The map of fundamental groups from $H$ to $\operatorname{Sp}(4) / \mathbb{Z}_{2}$ is surjective, so a surface operator with monodromy $S^{\prime}$ detects topology. On the other hand, the center of $\operatorname{Sp}(4)$ is generated by the element $-1 . S^{\prime}$ can be conjugated to $-S^{\prime}$ by an element $T^{\prime}$ which in $2 \times 2$ blocks looks like

$$
T^{\prime}=\left(\begin{array}{ll}
0 & 1 \\
1 & 0
\end{array}\right)
$$

and this shows that a surface operator with monodromy $S^{\prime}$ does not detect the center of $\operatorname{Sp}(4)$.

Since duality exchanges the center with the fundamental group, cf. (4.22), the fact that the unipotent surface operators of this class detect only the center while the semisimple ones detect only the topology is consistent with the proposal that $S$-duality exchanges these two kinds of surface operator. Another indication of this will emerge in Section 7 when we study quantization.

\subsection{Duality for $G=\mathrm{SO}(8)$}

Another instructive example, in which duality exchanges rigid surface operators in a far from obvious way, is the self-dual theory with gauge group $G=\mathrm{SO}(8)$. (Of course, $\mathrm{SO}(8)$ is self-dual only to the extent that the difference between the adjoint and simply connected forms is not essential.) In this case, in addition to the two rigid unipotent conjugacy classes of dimension 10 and 16 that we listed in the end of Section 2.3, we also have a 16-dimensional conjugacy class of a strongly rigid semisimple element

$$
S=\operatorname{diag}(-1,-1,-1,-1,+1,+1,+1,+1)
$$

which corresponds to the gauge symmetry breaking pattern $D_{2} \times D_{2} \subset D_{4}$. We list all of these conjugacy classes in the following table: 


\begin{tabular}{cc}
\hline$D_{4}$ & $\operatorname{dim}$ \\
\hline $\mathfrak{C}_{\left(\emptyset,\left[2^{2}, 1^{4}\right]\right)}^{D_{4}}$ & 10 \\
$\mathfrak{C}_{(\emptyset,[3,2,2,1])}^{D_{4}}$ & 16 \\
$\mathfrak{C}_{\left(\left[1^{4}\right],\left[1^{4}\right]\right)}^{D_{2} \times D_{2}}$ & 16 \\
\hline
\end{tabular}

This is the complete list of surface operators associated with strongly rigid non-central conjugacy classes for $G=\mathrm{SO}(8)$. While the surface operator associated with the ten-dimensional class is clearly self-dual (if only these strongly rigid classes are relevant), rigid surface operators associated with the two 16-dimensional conjugacy classes potentially can be mapped into each other. In particular, they have identical sets of polar polynomials. (The calculation of polar polynomials is similar to the example of Section 4.2.) Moreover, using the techniques of Section 4.3, we find that the surface operator associated with the rigid unipotent conjugacy class $\mathfrak{C}_{(\emptyset,[3,2,2,1])}^{D_{4}}$ can detect the center, but not the topology, of the gauge group. On the other hand, the surface operator associated with the rigid semisimple conjugacy class $\mathfrak{C}_{\left(\left[1^{4}\right],\left[1^{4}\right]\right)}^{D_{2} \times D_{2}}$ can detect the fundamental group, but not the center, of the gauge group, as expected for the dual surface operator.

Further evidence for the duality action on the three rigid surface operators listed here will become clear in the following sections. Thus, the unipotent conjugacy class labeled by $\lambda=\left[2^{2}, 1^{4}\right]$ is special and, as we explain in Section 7 , should map into a unipotent conjugacy class. On the other hand, the unipotent conjugacy class labeled by $\lambda=[3,2,2,1]$ is not special and, in general, duality maps such conjugacy classes into operators whose monodromy is not unipotent.

\subsection{Duality for $G=\mathrm{SO}(7)$ and ${ }^{L_{G}}=\mathrm{Sp}(6)$}

While two of our previous examples were rather subtle, the duality between gauge theories with $G=\mathrm{SO}(7)$ and ${ }^{L} G=\mathrm{Sp}(6)$ is very simple in a sense that dual pairs of strongly rigid surface operators in this case can be identified simply by comparing the most basic invariant, namely the dimension of the corresponding conjugacy classes.

In both gauge theories, the construction based on $\mathfrak{s u}(2)$ embeddings and Nahm's equations gives only one strongly rigid surface operator. In gauge theory with $G=\mathrm{SO}(7)$, this is a strongly rigid surface operators associated with a rigid nilpotent orbit labeled by $\lambda=[2,2,1,1,1]$. Similarly, in the dual theory with ${ }^{L} G=\mathrm{Sp}(6)$, there is one rigid surface operator associated 
with a strongly rigid nilpotent orbit labeled by $\lambda=[2,1,1,1,1]$ (see table in Section 2.3). These nilpotent orbits have dimensions 8 and 6 , respectively, which makes it clear that the construction of rigid surface operators based on $\mathfrak{s u}(2)$ embeddings and Nahm's equations is not sufficient for producing a set of surface operators closed under duality.

This situation is rectified if we include surface operators which correspond to strongly rigid semisimple conjugacy classes. In the theory with $G=\mathrm{SO}(7)$, there are two such surface operators, which correspond to rigid semisimple conjugacy classes $\mathfrak{C}_{\left(\left[1^{6}\right],[1]\right)}^{D_{3}}$ and $\mathfrak{C}_{\left(\left[1^{1}\right],\left[1^{3}\right]\right)}^{D_{2} \times B_{1}}$ of dimension 6 and 12, respectively. On the other hand, in the dual theory with ${ }^{L} G=\operatorname{Sp}(6)$, there are two surface operators that correspond to rigid semisimple conjugacy classes $\mathfrak{C}_{\left(\left[1^{2}\right],\left[1^{4}\right]\right)}^{C_{1} \times C_{2}}$ and $\mathfrak{C}_{([2,1,1],[1,1])}^{C_{2} \times C_{1}}$ of dimension 8 and 12 , respectively. Now, the complete list of strongly rigid surface operators has a nice form:

\begin{tabular}{ccc}
\hline$B_{3}$ & $\operatorname{dim}$ & $C_{3}$ \\
\hline $\mathfrak{C}_{\left(\left[1_{3}\right],[1]\right)}^{D_{3}}$ & 6 & $\mathfrak{C}_{\left(\emptyset,\left[2,1^{4}\right]\right)}^{C_{3}}$ \\
$\mathfrak{C}_{\left(\emptyset,\left[2,2,1^{3}\right]\right)}^{B_{3}}$ & 8 & $\mathfrak{C}_{\left(\left[1^{2}\right],\left[1^{4}\right]\right)}^{C_{1}}$ \\
$\mathfrak{C}_{\left(\left[1^{1}\right],\left[1^{3}\right]\right)}^{D_{2} \times B_{1}}$ & 12 & $\mathfrak{C}_{([2,1,1],[1,1])}^{C_{2} \times C_{1}}$ \\
\hline
\end{tabular}

In each case, we find three strongly rigid conjugacy classes of the same dimension, which allows to identify unambiguously dual pairs of rigid surface operators for $G=\mathrm{SO}(7)$ and ${ }^{L} G=\operatorname{Sp}(6)$. As a strong test of the duality, we have checked that all other invariants of dual surface operators also match. In particular, in the previous section we already gave a detailed comparison of the polar polynomials for the six-dimensional conjugacy classes $\mathfrak{C}_{\left(\left[1^{6}\right],[1]\right)}^{D_{3}}$ and $\mathfrak{C}_{\left(\emptyset,\left[2,1^{4}\right]\right)}^{C_{3}}$.

\subsection{Duality for $G=\operatorname{SO}(9)$ and ${ }^{L} G=\operatorname{Sp}(8)$}

Now we turn to $\mathrm{SO}(9)$ and $\mathrm{Sp}(8)$. This turns out to be the first case in which we do not get a consistent picture in considering only strongly rigid conjugacy classes. Possibly, this means that for these groups, a full duality statement involves also the more delicate constructions that were described for $\mathrm{SU}(2)$ and $\mathrm{SO}(3)$ in Section 3.4. (Of course, these constructions may also be relevant for a more complete treatment of the groups that we have just considered.)

As in the previous examples, we start with rigid surface operators constructed via $\mathfrak{s u}(2)$ embeddings $\rho: \mathfrak{s u}(2) \rightarrow \mathfrak{g}$. These surface operators 
correspond to rigid unipotent conjugacy classes which, for $B_{4}$ and $C_{4}$, we summarized in the table in the end of Section 2.3. Namely, in $B_{4}$ there are two rigid unipotent conjugacy classes labeled by $\lambda=\left[2,2,1^{5}\right]$ and $\lambda=\left[2^{4}, 1\right]$. Similarly, in $C_{4}$ there are also two rigid unipotent conjugacy classes labeled by $\lambda=\left[2,1^{6}\right]$ and $\lambda=\left[2^{3}, 1^{2}\right]$. However, these rigid unipotent conjugacy classes have completely different dimensions which, again, makes it clear that $S$-duality cannot work unless we enlarge the set of rigid surface operators at least by including those corresponding to strongly rigid semisimple conjugacy classes. Once we do this, the list of strongly rigid surface operators in $B_{4}$ and $C_{4}$ becomes considerably larger, with some obvious matches:

\begin{tabular}{c|c|c}
\hline$B_{4}$ & $\operatorname{dim}$ & $C_{4}$ \\
\hline $\mathfrak{C}_{\left(\left[1^{8}\right],[1]\right)}^{D_{4}}$ & 8 & $\mathfrak{C}_{\left(\emptyset,\left[2,1^{6}\right]\right)}^{C_{4}}$ \\
$\mathfrak{C}_{\left(\emptyset,\left[2,2,1^{5}\right]\right)}^{B_{4}}$ & 12 & $\mathfrak{C}_{\left(\left[1^{2}\right],\left[1^{6}\right]\right)}^{C_{1} \times C_{3}}$ \\
$\mathfrak{C}_{\left(\emptyset,\left[2^{4}, 1\right]\right)}^{B_{4}}$ & 16 & $\mathfrak{C}_{\left(\left[1^{4}\right],\left[1^{4}\right]\right)}^{C_{2} \times C_{2}}$ \\
$\mathfrak{C}_{\left(\left[1^{6}\right],\left[1^{3}\right]\right)}^{D_{3} \times B_{1}}$ & 18 & $\mathfrak{C}_{\left(\emptyset,\left[2^{3}, 1^{2}\right]\right)}^{C_{4}}$ \\
$\mathfrak{C}_{\left(\left[2^{2}, 1^{4}\right],[1]\right)}^{D_{4}}$ & 18 & $\mathfrak{C}_{\left(\left[1^{2}\right],\left[2,1^{4}\right]\right)}^{C_{1} \times C_{3}}$ \\
$\mathfrak{C}_{\left(\left[1^{4}\right],\left[1^{5}\right]\right)}^{D_{2} \times B_{2}}$ & 20 & $\mathfrak{C}_{\left(\left[2,1^{2}\right],\left[1^{4}\right]\right)}^{C_{2} \times C_{2}}$ \\
$\mathfrak{C}_{([3,2,2,1],[1])}^{D_{4}}$ & 24 & $\mathfrak{C}_{\left(\left[2,1^{2}\right],\left[2,1^{2}\right]\right)}^{C_{2} \times C_{2}}$ \\
$\mathfrak{C}_{\left(\left[1^{4}\right],[2,2,1]\right)}^{D_{2} \times B_{2}}$ & 24 & $?$ \\
\hline
\end{tabular}

In particular, strongly rigid surface operators that correspond to conjugacy classes of dimension $8,12,16$, and 20 can be identified simply by matching the dimension. For these surface operators, one can also check that all other invariants match, in complete agreement with the duality.

Strongly rigid surface operators that correspond to conjugacy classes of dimension 18 and 24 are more interesting. In dimension 18, there is an ambiguity in the matching that is actually not resolved by our other invariants. 
All four surface operators of dimension 18 listed in the table have the same set of polar polynomials:

$$
\begin{aligned}
\operatorname{Tr} \varphi^{2} & \simeq \frac{p_{2}}{z}+\cdots \\
\operatorname{Tr} \varphi^{4} & \simeq \frac{p_{4}}{z^{2}}+\frac{p_{1}}{z}+\cdots \\
\operatorname{Tr} \varphi^{6} & \simeq \frac{p_{6}}{z^{3}}+\frac{p_{3}}{z^{2}}+\frac{p_{5}}{z}+\cdots \\
\operatorname{Tr} \varphi^{8} & \simeq \frac{p_{8}}{z^{4}}+\frac{p_{7}}{z^{3}}+\frac{p_{9}}{z^{2}}+\frac{p_{10}}{z}+\cdots
\end{aligned}
$$

Since these orbits have dimension 18, we expect the space of polar polynomials to be nine dimensional. Therefore, we expect one relation among the ten parameters $p_{i}$. This relation turns out to be

$$
p_{8}=\frac{1}{48} p_{2}^{4}-\frac{1}{4} p_{2}^{2} p_{4}+\frac{1}{4} p_{4}^{2}+\frac{2}{3} p_{2} p_{6}
$$

(which one can verify to be invariant under reparametrization of the local coordinate $z$ ). Moreover, even the discrete invariants of Section 4.3 do not help to resolve the ambiguity in matching of orbits. Indeed, in $B_{4}$, both rigid surface operators associated with the 18-dimensional conjugacy classes $\mathfrak{C}_{\left(\left[1^{6}\right],\left[1^{3}\right]\right)}^{D_{3} \times B_{1}}$ and $\mathfrak{C}_{\left(\left[2^{2}, 1^{4}\right],[1]\right)}^{D_{4}}$ can detect the fundamental group $\pi_{1}(G)$, but not the center $\mathcal{Z}(G)$. On the other hand, in $C_{4}$, both rigid surface operators associated with the 18-dimensional conjugacy classes $\mathfrak{C}_{\left(\emptyset,\left[2^{3}, 1^{2}\right]\right)}^{C_{4}}$ and $\mathfrak{C}_{\left(\left[1^{2}\right],\left[2,1^{4}\right]\right)}^{C_{1} \times C_{3}}$ can detect the center $\mathcal{Z}\left({ }^{L} G\right)$, but not the fundamental group $\pi_{1}\left({ }^{L} G\right)$. This is consistent with the duality, but one needs finer invariants in order to say more precisely how these 18-dimensional conjugacy classes are paired up.

In dimension 24, we have a worse problem: there are two strongly rigid conjugacy classes in $B_{4}$ and only one in $C_{4}$ so there is no hope of matching them. Perhaps the inclusion of more delicate constructions of surface operators - such as those of Section 3.4 - is needed for resolving this problem.

Still, it is attractive that all but one strongly rigid surface operators in our table does appear to have a dual.

\section{More examples}

Although we do not know the general mapping from rigid surface operators in a theory with gauge group $G$ to similar operators in the dual theory with 
gauge group ${ }^{L} G$, in this section we make a duality conjecture for certain infinite families of surface operators. The proposal generalizes examples seen in the previous section. Again, our main tools for identifying dual pairs will be invariants described in Section 4 .

\subsection{Special rigid orbits}

As we have already mentioned, there is no bijection between nilpotent orbits (rigid or not) for the dual groups $G_{\mathbb{C}}$ and ${ }^{L} G_{\mathbb{C}}$. There is, however, a nice bijection between a certain subset of nilpotent orbits called special orbits ${ }^{13}$ which has been studied in the mathematical literature [6,7] (see also [18]). This bijection is defined by considering representations of the Weyl group associated to an orbit by the Springer correspondence, while we are interested in a duality map that preserves the invariants of Section 4. The most important invariant in what follows is the conjugacy class in the Weyl group associated to an orbit by the Kazhdan-Lusztig map; this is somewhat analogous to the invariant associated with the Springer correspondence.

Many special orbits are not rigid; however, some of them are. And even though explaining how generic special orbits transform under duality involves a rather sophisticated combinatorics, the case of surface operators associated with special rigid orbits is considerably easier. First, we will give an idea of what special orbits look like, and then specialize to the rigid ones.

There are several ways to define special orbits. For example, one definition, related to quantization, will be mentioned in Section 7. Here, we present another, equivalent definition which is helpful for better understanding of the set of nilpotent orbits (or unipotent conjugacy classes) as a whole. We have seen in Section 2.2, for the example of $G=\mathrm{SU}(2)$, that it is possible for one nilpotent orbit (the orbit of the zero element of $\mathfrak{s l}(2)$ ) to be in the closure of another (the orbit of a regular nilpotent element). Exploiting this idea, we get a natural partial order on the set of nilpotent orbits. Let $\mathfrak{c}_{\lambda}$ and $\mathfrak{c}_{\mu}$ be two nilpotent orbits. One says that $\mathfrak{c}_{\lambda} \leq \mathfrak{c}_{\mu}$ if $\mathfrak{c}_{\lambda}$ is contained in the closure of $\mathfrak{c}_{\mu}$, that is if $\mathfrak{c}_{\lambda} \subset \overline{\mathfrak{c}}_{\mu}$. For classical groups, we can think of $\lambda$ and $\mu$ as partitions, $N=\lambda_{1}+\cdots+\lambda_{n}=\mu_{1}+\mu_{2}+\cdots+\mu_{n^{\prime}}$ (where we

\footnotetext{
${ }^{13}$ Special nilpotent orbits include Richardson orbits, which correspond in the following sense to surface operators studied in [2] and reviewed in Section 2.1. Let $\mathbb{L}$ be a Levi subgroup of $G$, with $\mathbb{L}$-regular parameters $\alpha, \beta, \gamma$. In the limit that these parameters are all taken to zero, the monodromy of the surface operator generically takes values in the Richardson unipotent orbit associated to $\mathbb{L}$. For more on this see Section 3.3 of [2].
} 


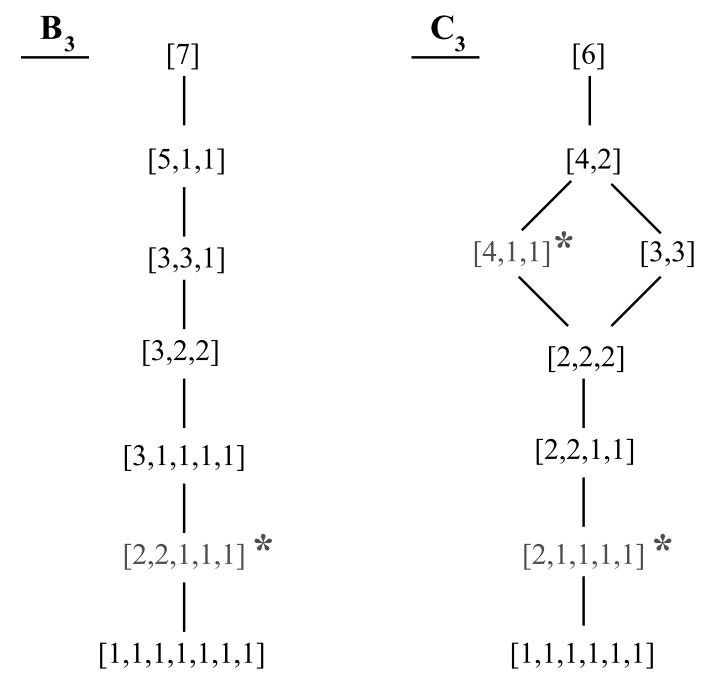

Figure 2: Hasse diagram for $B_{3}$ and $C_{3}$. If $\lambda \leq \mu$, then $\lambda$ is shown below $\mu$ in the diagram. Nilpotent orbits that are not special are shown in red and labeled by an asterisk; omitting such orbits gives a diagram with an order-reversing involution.

take $\lambda_{i} \geq \lambda_{j}, \mu_{i} \geq \mu_{j}$ for $j>i$ ). We say that $\lambda \leq \mu$ if

$$
\sum_{i=1}^{k} \lambda_{i} \leq \sum_{i=1}^{k} \mu_{i}
$$

for all $k$. This condition is equivalent with one exception ${ }^{14}$ that will not concern us to the condition $\mathfrak{c}_{\lambda} \leq \mathfrak{c}_{\mu}$. For example, the closure ordering of nilpotent orbits in $B_{3}$ and $C_{3}$ can be summarized in figure 2 .

There is a natural order-reversing involution on the set of nilpotent orbits, which in type $A$ corresponds to a map $\lambda \rightarrow \lambda^{\mathrm{t}}$, where $\lambda^{\mathrm{t}}$ is the transpose partition of $\lambda$, see, e.g., [12]. The transpose partition is described as follows. We relate a partition to a Young tableau by turning every "part" $\lambda_{i}$ into a column of height $\lambda_{i}$. For example, for $N=11$, to the partition $N=$ $3+3+2+2+1$, we associate the Young tableau

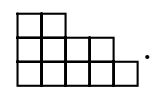

\footnotetext{
${ }^{14}$ The exception arises for $D_{n}$ in the case of a very even partition, that is a partition such that the $\lambda_{i}$ are all even. Such a partition corresponds to two distinct nilpotent orbits, neither of which is in the closure of the other.
} 
To make the transpose partition, we just take the transpose of the picture. Thus, for the example that we just gave, the transpose operation is



so the transpose of the partition $\left[3^{2}, 2^{2}, 1\right]$ is $[5,4,2]$.

For a group of type $A$, the transpose operation makes sense for any partition. It can be shown that it reverses order, meaning that if $\lambda \leq \mu$, then $\mu^{\mathrm{t}} \leq \lambda^{\mathrm{t}}$.

For other classical groups $B, C$, and $D$, the transpose operation does not make sense for an arbitrary partition. For these groups, nilpotent orbits are associated with partitions that obey certain constraints (the constraints are stated at the end of Section 2.2). These constraints are not invariant under the transpose operation. For example, in the $B$ case, the constraint is that if $\lambda_{i}$ is even, it must occur with even multiplicity. In the example of equation (6.2), we see that $\lambda=\left[3^{2}, 2^{2}, 1\right]$ obeys this constraint, and $\lambda^{\mathrm{t}}=$ $[5,4,2]$ does not.

For groups of type $B$, and $C$, we say that a partition $\lambda$ is special (or the corresponding orbit $\mathfrak{c}_{\lambda}$ is special) if the transpose $\lambda^{\mathrm{t}}$ obeys the relevant conditions (for the same group). Thus, in the above example, $\lambda$ is not special. On the other hand, for $\mathrm{SO}(9)$ the partition $\left[3,2^{2}, 1^{2}\right]$ is special since its transpose, which is $[5,3,1]$, has no even parts:

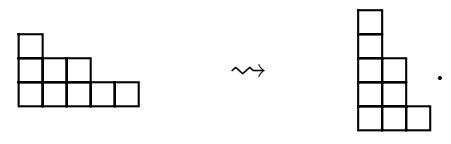

For type $A$, all nilpotent orbits are special since $\lambda$ is subject to no constraint. For groups of type $D$, a nilpotent orbit $\mathfrak{c}_{\lambda}$ labeled by an orthogonal partition $\lambda$ is special if and only if the transpose partition $\lambda^{\mathrm{t}}$ is symplectic. For example, the orbit labeled by $\lambda=[3,2,2,1]$ in $D_{4}$ that we discussed in Section 5.2 is not special since the transpose partition $\lambda^{t}=[4,3,1]$ is not symplectic:

$$
\lambda=\prod_{\square} \quad \rightsquigarrow \quad \lambda^{t}=\square_{\square} .
$$

This definition is rather surprising, but we will not describe it here, as we will not go into any depth concerning groups of type $D$. 
Now we focus on groups of type $B$ and $C$. Clearly, with the above definition, the operation $\lambda \rightarrow \lambda^{\mathrm{t}}$ makes sense for special partitions. Since we are interested in rigid surface operators, our next task is to single out those special partitions $\lambda$ for which $\mathfrak{c}_{\lambda}$ is rigid. First, let us consider $B_{N}$. As usual, we label nilpotent orbits by partitions

$$
\lambda=\left[k^{n_{k}}(k-1)^{n_{k-1}} \cdots 2^{n_{2}} 1^{n_{1}}\right],
$$

where we assume that the multiplicities $n_{i}$ are positive precisely if $i \leq k$. (This is one of the criteria for rigidness that were described in Section 2.3.) Of course, we also have $n_{2 i}$ even since we are considering orbits in $B_{N}$. Finally, the other criterion for rigidness is that $n_{2 i+1} \neq 2$ for all $i$. Imposing an extra condition that the orbit is special, one finds that $n_{k}$ is odd and $n_{i}$ is even for all $i<k$. For example, since $n_{k-1} \neq 0$, we find that the transpose partition has for its smallest part the number $n_{k}$ occurring with multiplicity 1 , as in this example:

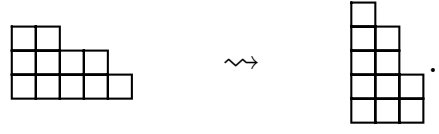

Hence, if $n_{k}$ is even (as in this example) then $\lambda^{\mathrm{t}}$ is not orthogonal. A similar argument shows that $n_{i}$ must be even for $i<k$.

Therefore, we conclude that special rigid orbits in $B_{N}$ are associated with partitions of the form

$$
\lambda=\left[k^{2 m_{k}+1}(k-1)^{2 m_{k-1}} \cdots 2^{m_{2}} 1^{2 m_{1}}\right]
$$

with $k$ odd.

Similarly, a rigid orbit in $C_{N}$ is labeled by the partition in the form (6.5) with $n_{2 i+1}$ even and $n_{2 i}$ not equal to 2 . In addition, such an orbit is special if all $n_{i}$ are even. Hence, we label special rigid orbits in $C_{N}$ by

$$
\lambda=\left[k^{2 m_{k}}(k-1)^{2 m_{k-1}} \cdots 2^{2 m_{2}} 1^{2 m_{1}}\right] .
$$

Notice that in the case of $C_{N}$ we do not need to assume that $k$ is odd.

Now, let us identify dual pairs of special rigid surface operators. Starting with a special rigid orbit labeled by the symplectic partition (6.8) of $2 N$, we need to describe the orthogonal partition of $2 N+1$ that labels the dual orbit. It can be constructed by the following simple rule: ${ }^{15}$ in the symplectic

\footnotetext{
${ }^{15}$ This rule was found by comparing to some constructions of Lusztig [7] as well as to examples in the last section.
} 
partition, every block $l^{n_{l}}$ with $l$ odd remains invariant, while in every block $l^{n_{l}}$ with $l$ even one of the parts is replaced by $l+1$ and one other part is replaced by $l-1$ :

$$
l^{n_{l}} \mapsto \begin{cases}(l+1) l^{n_{l}-2}(l-1), & l \text { even, } \\ l^{n_{l}}, & l \text { odd } .\end{cases}
$$

Note that this operation does not change the net sum of all the parts. Hence, we also add " 1 " to the resulting partition in order to obtain a partition of $2 N+1$ (instead of a partition of $2 N$ ). In the end, we obtain the following $\operatorname{map}^{16}$

$$
\begin{aligned}
& {\left[k^{2 m_{k}} \cdots 3^{2 m_{3}} 2^{2 m_{2}} 1^{2 m_{1}}\right]} \\
& \quad \mapsto \begin{cases}{\left[(k+1)^{1} k^{2 m_{k}-2} \cdots 3^{2 m_{3}+2} 2^{2 m_{2}-2} 1^{2 m_{1}+2}\right],} & k \text { even } \\
{\left[k^{2 m_{k}+1}(k-1)^{2 m_{k-1}-2} \cdots 3^{2 m_{3}+2} 2^{2 m_{2}-2} 1^{2 m_{1}+2}\right],} & k \text { odd }\end{cases}
\end{aligned}
$$

where $m_{j}>1$ if $j$ is even, and $m_{j}>0$ in general.

This map preserves all the invariants of surface operators introduced in Section 4. For example, using (4.1) we find that special rigid orbits labeled by partitions (6.9) have the same dimension, given by

$$
\operatorname{dim}\left(\mathfrak{c}_{\lambda}\right)=2 N^{2}+N-2 \sum_{i}\left(\sum_{j \geq i} n_{j}\right)^{2}-\sum_{i \text { odd }} n_{i} .
$$

Similarly, one can identify the corresponding polar polynomials or, equivalently, the conjugacy class in the Weyl group $\mathcal{W}$ under the Kazhdan-Lusztig map. For groups of type $B_{N}$ and $C_{N}$ that we are considering here, conjugacy classes in $\mathcal{W}$ are indexed by pairs of partitions $\left(\nu_{+}, \nu_{-}\right)$such that $\left|\nu_{+}\right|+\left|\nu_{-}\right|=N$, see, e.g., [17]. In particular, $\left(\nu_{+}, \nu_{-}\right)=([1,1, \ldots, 1], \emptyset)$ corresponds to the class of the identity in $\mathcal{W}$, while $\left(\nu_{+}, \nu_{-}\right)=(\emptyset,[N])$ corresponds to the Coxeter class (the class which contains a cyclic permutation of order $N)$. After a somewhat lengthy calculation, using formulas in [17], one finds that under the Kazhdan-Lusztig map, dual orbits identified in (6.9) map to the same conjugacy class in $\mathcal{W}$, namely

$$
\left(\left[\cdots 5^{n_{5}} 3^{2 n_{3}} 1^{n_{1}}\right],\left[\cdots 3^{2 n_{6}} 2^{2 n_{4}} 1^{2 n_{2}}\right]\right) .
$$

Therefore, we conclude that special rigid orbits identified by (6.9) have the same fingerprints.

\footnotetext{
${ }^{16}$ Here $(k+1)^{1}$ refers to a part $k+1$ that appears with multiplicity 1.
} 
Finally, we consider the center and topology of $G$ and ${ }^{L} \neg G$ as follows. For a unipotent surface operator with gauge group $G$, one can always observe the center of $G$, as explained in Section 4.3. Dually, consider a surface operator in a theory with gauge group ${ }^{L} G$ and let $H$ be the automorphism group of this surface operator. Then one can observe the topology of ${ }^{L} G$ if the natural map $\iota: \pi_{1}(H) \rightarrow \pi_{1}\left({ }^{L} G\right)$ is surjective. For ${ }^{L} G$ of type $B_{N}$, this will be so if $H$ contains a factor $\mathrm{SO}(n), n \geq 2$, since the map of fundamental groups $\mathrm{SO}(2) \rightarrow \mathrm{SO}(2 N+1)$ is surjective. In turn, for a surface operator associated with a partition $\lambda, H$ has such a factor if one of the odd parts in $\lambda$ has multiplicity at least 2. According to (6.7), this is so whenever $\lambda$ is rigid and special. For ${ }^{L} G$ of type $C_{N}$, the condition we need is that the central element -1 of ${ }^{L} G$ should be connected to the identity in the subgroup $H$ that commutes with the embedding $\rho: \mathfrak{s l}(2) \rightarrow{ }^{L} \mathfrak{g}$. (A path from 1 to -1 in $C_{N}=\operatorname{Sp}(2 N)$ projects in $\operatorname{Sp}(2 N) / \mathbb{Z}_{2}$ to a generator of the fundamental group.) $H$ is a product of factors $H_{\lambda^{*}}$, associated respectively with the parts of size $\lambda^{*}$. If a part $\lambda^{*}$ appears with multiplicity $m$, then $H_{\lambda^{*}}$ is $\mathrm{SO}(m)$ or $\mathrm{Sp}(m)$ (depending on whether $\lambda^{*}$ is even or odd). The element -1 is connected to the identity in $\mathrm{SO}(m)$ or $\mathrm{Sp}(m)$ if $m$ is even, which is always true in the rigid special case, according to equation (6.8).

\subsection{Dualities involving rigid semisimple orbits}

Now let us consider dual pairs that involve rigid semisimple conjugacy classes on at least one side. Simple classes of this type were described in Section 2.4 .

We start with the minimal unipotent orbit in $C_{N}$ (whose dual turns out to be semisimple). We already discussed this orbit in detail in Section 2.3 , equations (2.25) and (2.26). In the notation of Section 2.5, this orbit corresponds to $\Theta_{0}=\Delta$ with $\lambda^{\prime}=\emptyset$ and $\lambda^{\prime \prime}=\left[2,1^{2 N-2}\right]$. It has dimension $2 N$ and via the Kazhdan-Lusztig map is identified with the following conjugacy class in the Weyl group:

$$
\left(\left[1^{N-1}\right],[1]\right) .
$$

In type $B_{N}$, there is also a $2 N$-dimensional conjugacy class $\mathfrak{C}_{\left(\left[1^{2 N}\right],[1]\right)}^{D_{N}}$ of the semisimple element

$$
S=\operatorname{diag}(1,-1,-1, \ldots,-1)
$$

which has the same behavior of the Higgs field and discrete invariants introduced in Section 4.3. This is a strong hint that the corresponding surface operators are dual. 
For our next example we take the strongly rigid unipotent conjugacy class in $B_{N}$ corresponding to the partition $\lambda^{\prime \prime}=\left[2,2,1^{2 N-3}\right]$. This conjugacy class was also discussed in Section 2.3. It has dimension $4(N-1)$ and via the Kazhdan-Lusztig map is identified with the following conjugacy class in the Weyl group:

$$
\left(\left[2,1^{N-2}\right], \emptyset\right) \text {. }
$$

One finds the same behavior of the Higgs field for the strongly rigid conjugacy class of the semisimple element

$$
S=\operatorname{diag}(1,1,-1,-1, \ldots,-1)
$$

in $C_{N}$ associated with $\Theta_{1}=C_{1} \times C_{N-1}$ and $\left(\lambda^{\prime}, \lambda^{\prime \prime}\right)=\left(\left[1^{2}\right],\left[1^{2 N-2}\right]\right)$.

Our next example involves dual pairs of surface operators, both of which correspond to strongly rigid semisimple conjugacy classes. In type $B_{N}$, we consider the conjugacy class associated to $\Theta_{2}=D_{2} \times B_{N-2}$ and $\left(\lambda^{\prime}, \lambda^{\prime \prime}\right)=$ ( $\left.\left[1^{4}\right],\left[1^{2 N-3}\right]\right)$. On the other hand, in type $C_{N}$ the candidate for the dual conjugacy class has $\Theta_{2}=C_{2} \times C_{N-2}$ and $\left(\lambda^{\prime}, \lambda^{\prime \prime}\right)=\left([2,1,1],\left[1^{2 N-4}\right]\right)$. It is possible to check that both of these conjugacy classes have equal polar polynomials and their image under the Kazhdan-Lusztig map is the same conjugacy class in the Weyl group:

$$
\left(\emptyset,\left[1^{N}\right]\right) .
$$

Summarizing, we find the following families of dual pairs of rigid semisimple surface operators:

\begin{tabular}{cc}
\hline \multicolumn{1}{c}{$B_{N}$} & $C_{N}$ \\
\hline $\mathfrak{C}_{\left(\left[1^{2 N}\right],[1]\right)}^{D_{N}}$ & $\mathfrak{C}_{\left(\emptyset,\left[2,1^{2 N-2}\right]\right)}^{C_{N}}$ \\
$\mathfrak{C}_{\left(\emptyset,\left[2,2,1^{2 N-3}\right]\right)}^{B_{N}}$ & $\mathfrak{C}_{\left(\left[1^{2}\right],\left[1^{2 N-2}\right]\right)}^{C_{1} \times C_{N-1}}$ \\
$\mathfrak{C}_{\left(\left[1^{4}\right],\left[1^{2 N-3}\right]\right)}^{D_{2} \times B_{N-2}}$ & $\mathfrak{C}_{\left([2,1,1],\left[1^{2 N-4}\right]\right)}^{C_{2} \times C_{N-2}}$ \\
$\ldots$ & $\ldots$ \\
\hline
\end{tabular}

We note that these examples completely cover all dual pairs of strongly rigid conjugacy classes in small rank $N \leq 3$. In particular, for $N=2$ we recover a somewhat subtle duality between rigid surface operators in $B_{2}$ and $C_{2}$ discussed in Section 5.1.

Lusztig in [8, Section 13.3], generalizes the correspondence between special unipotent classes in $G_{\mathbb{C}}$ and ${ }^{L} G_{\mathbb{C}}$ to a surjective map from special conjugacy classes in $G_{\mathbb{C}}$ that are not necessarily unipotent to unipotent classes in 
${ }^{L} G_{\mathbb{C}}$ that are not necessarily special. The first two entries in the above table appear to be special cases of this definition, although the third is not. The table is hopefully an approximation to a more complete table that would describe a bijection between suitable objects on the two sides.

\section{$7 \quad$ Duality and quantization}

In this paper, we have mainly studied "static" half-BPS surface operators supported on $D=\mathbb{R}^{2}$ in the space-time manifold $M=\mathbb{R}^{4}$. This problem admits various generalizations; in particular, one can consider more general space-time manifolds $M$ and embedded surfaces $D \subset M$, including those with boundary. A simple example of such a generalization is obtained by taking

$$
M=\mathbb{R}^{3} \times[0,1]
$$

with supersymmetric boundary conditions $\mathcal{B}_{ \pm}$at $W_{-}=\mathbb{R}^{3} \times\{0\}$ and $W_{+}=$ $\mathbb{R}^{3} \times\{1\}$, and with a "static" surface operator on $D=\mathbb{R} \times[0,1]$, where $\mathbb{R} \subset \mathbb{R}^{3}$ stands for the "time" direction, parametrized by $x^{0}$. (see figure 3 ).

Quantization of this theory gives a Hilbert space, $\mathcal{H}$, which depends on all the choices involved, in particular, on the surface operator as well as on the boundary conditions $\mathcal{B}_{+}$and $\mathcal{B}_{-}$. As will be explained in more detail elsewhere [19], for a suitable choice of boundary conditions $\mathcal{B}_{ \pm}$and a surface operator on $D=\mathbb{R} \times[0,1]$, the space $\mathcal{H}$ is a representation of a real form $G_{\mathbb{R}}$ of the complexified gauge group $G_{\mathbb{C}}$.

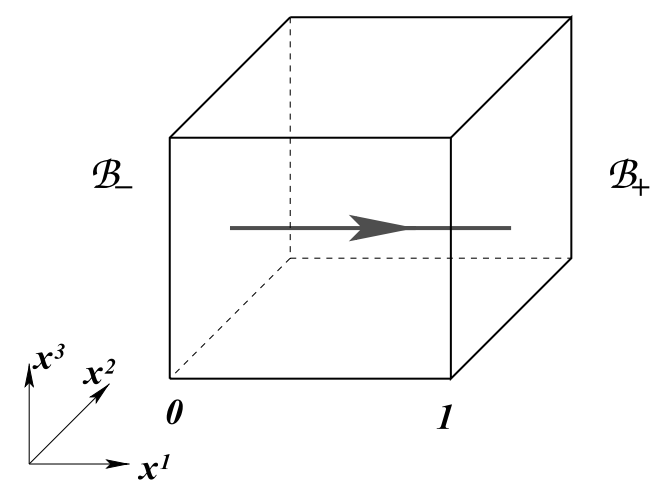

Figure 3: A time zero slice of a time-independent configuration on $M=$ $\mathbb{R}^{3} \times[0,1]$ with boundary conditions $\mathcal{B}_{+}$and $\mathcal{B}_{-}$. The support $D$ of a surface operator intersects the time zero slice on the interval $I=[0,1]$, parametrized by $x^{1}$. 
In this construction, we consider a surface operator associated with a unipotent conjugacy class $\mathfrak{C}$ or with a semisimple conjugacy class obtained by a deformation of $\mathfrak{C}$. Furthermore, the real form $G_{\mathbb{R}}$ is determined by one of the boundary conditions, say $\mathcal{B}_{-}$, while the other boundary condition, $\mathcal{B}_{+}$, is universal. In compactification to two dimensions, $\mathcal{B}_{+}$corresponds to the so-called canonical coisotropic brane; see [20, Section 12.4] for a detailed description of this boundary condition in four-dimensional gauge theory. In particular, $\mathcal{B}_{+}$includes mixed Neumann-Dirichlet boundary conditions for the gauge field $A$ and the Higgs field $\phi$ :

$$
\begin{aligned}
D_{0} \phi_{2}+\partial_{1} A_{2} & =0, \\
D_{0} \phi_{3}+\partial_{1} A_{3} & =0, \\
F_{02}-\partial_{1} \phi_{2} & =0, \\
F_{03}-\partial_{1} \phi_{3} & =0,
\end{aligned}
$$

where $x^{1}$ is the coordinate on the interval $[0,1]$.

For applications to the present paper, the details of the second boundary condition $\mathcal{B}_{-}$are not important, as long as it preserves the same supersymmetry $^{17}$ as $\mathcal{B}_{+}$. A particularly nice class of boundary conditions $\mathcal{B}_{-}$ correspond to imposing Dirichlet boundary conditions on half of the fields $(A, \phi)$. This can be done so that the boundary conditions, upon reduction to the sigma model, define a Lagrangian brane supported on a $G_{\mathbb{R}}$-conjugacy class $\mathfrak{C}_{\mathbb{R}} \subset \mathfrak{C}$, for some real form $G_{\mathbb{R}}$ of $G_{\mathbb{C}}$. For example, it is easy to see that the Dirichlet boundary condition

$$
\mathcal{B}_{-}:\left.\quad \phi\right|_{W_{-}}=0
$$

restricts the monodromy $V$ of the connection $\mathcal{A}=A+\mathrm{i} \phi$ to be in a conjugacy class of the compact group $G$. More generally, one can define a boundary condition $\mathcal{B}_{-}$associated with a $G_{\mathbb{R}}$-conjugacy class $\mathfrak{C}_{\mathbb{R}}$ for some real form $G_{\mathbb{R}}$ of $G_{\mathbb{C}}$, such that

$$
V \in \mathfrak{C}_{\mathbb{R}}
$$

For our purposes, all we need to know from [19] is that the central character $\zeta$ of the representation $\mathcal{H}$ attached to $\mathfrak{C}_{\mathbb{R}}$ depends only on the surface operator (that is, on the corresponding conjugacy class $\mathfrak{C}$ ) involved in this construction and not on the particular choice of the boundary condition $\mathcal{B}_{-}$ (which, among other things, determines the real form $G_{\mathbb{R}}$ and $\mathfrak{C}_{\mathbb{R}} \subset \mathfrak{C}$ ). In

\footnotetext{
${ }^{17}$ In fact, it is really only necessary to preserve part of their common supersymmetry. The important part is the supersymmetry of the relevant $A$-model.
} 
particular, for a surface operator (2.4) labeled by a Levi subgroup $\mathbb{L}$ and continuous parameters $(\alpha, \beta, \gamma, \eta)$, the central character is given by [19]

$$
\zeta=\eta+\mathrm{i} \gamma
$$

More generally, in all examples that we have checked of surface operators constructed via $\mathfrak{s u}(2)$ embeddings and solutions to Nahm's equations, it turns out that the central character $\zeta$ of the $G_{\mathbb{R}}$-representation attached to $\mathfrak{C}_{\mathbb{R}} \subset \mathfrak{C}$ is related to the semisimple part ${ }^{L} S$ of the conjugacy class ${ }^{L_{\mathfrak{C}}}$ associated with the dual surface operator,

$$
{ }^{L_{S}}=\exp (2 \pi \zeta)
$$

This motivates the following conjecture:

Conjecture. Let $\mathfrak{C}$ be a unipotent conjugacy class (or a semisimple conjugacy class obtained by a deformation of $\mathfrak{C})$. Then, the parameter $\frac{1}{2 \pi} \log { }^{L} S$ of the dual conjugacy class ${ }^{L_{\mathfrak{C}}}$ is equal to the central character $\zeta$ of (any) $G_{\mathbb{R}}$-representation attached to $\mathfrak{C}_{\mathbb{R}} \subset \mathfrak{C}$.

This conjecture can be verified for many surface operators. In particular, it holds for all the surface operators constructed in [2] and reviewed in Section 2.1. Indeed, let us consider a surface operator (2.4) labeled by a Levi subgroup $\mathbb{L}$ and continuous parameters $(\alpha, \beta, \gamma, \eta)$. As explained in [19] and summarized in equation (7.4), in this case the central character is $\zeta=\eta+\mathrm{i} \gamma$. Since $\eta$ is a "quantum" parameter, it is convenient to use the duality transformation of the parameters of such surface operators [2],

$$
(\alpha, \eta) \rightarrow(\eta,-\alpha)
$$

to write $\zeta$ in terms of "classical" parameters in the dual theory:

$$
\zeta=-{ }^{L} \alpha+i^{L} \gamma .
$$

This indeed equals the semisimple part ${ }^{L} S$ of the dual conjugacy class ${ }^{L} \mathfrak{C}$, thus justifying (7.5) for surface operators associated with Richardson conjugacy classes and their semisimple deformations. However, one can verify the above conjecture for more general surface operators, including rigid unipotent surface operators studied in this paper.

A simple class of surface operators that are rigid (and, therefore, not included in those of [2]) can be constructed in a theory with gauge group $G=\operatorname{Sp}(2 N)$ via $\mathfrak{s u}(2)$ embeddings labeled by $\lambda=\left[2,1^{2 N-2}\right]$. We already 
considered such rigid surface operators in the previous sections; they correspond to minimal orbits $\mathfrak{c}_{\min }$ in $C_{N}$. The minimal orbit $\mathfrak{c}_{\min }$ in $C_{N}$ has dimension $2 N$, and the representation attached to this orbit is the familiar Weyl representation obtained by quantizing the phase space of $N$ decoupled harmonic oscillators. (For mathematical literature on quantization of the minimal orbit $\mathfrak{c}_{\min }$, see, e.g., $[21,22]$.) The central character of this representation gives a rigid semisimple element

$$
{ }^{L_{S}}=\exp (2 \pi \zeta)=\operatorname{diag}(+1,-1,-1, \ldots,-1)
$$

in the dual group, ${ }^{L} G=\mathrm{SO}(2 N+1)$. It corresponds to the root system of type $D_{N}$, and its conjugacy class $\mathfrak{C}_{L_{S}}$ also has dimension $2 N$. In fact, $\mathfrak{C}_{L_{S}}=\mathfrak{C}_{\left(\left[1^{2 N}\right],[1]\right)}^{D_{N}}$ is precisely the rigid semisimple conjugacy class of a surface operator in the ${ }^{L} G=\mathrm{SO}(2 N+1)$ theory that, by matching invariants, we proposed in Section 6.2 as the dual to the rigid surface operator associated with the minimal nilpotent orbit $\mathfrak{c}_{\min }$ in the $G=\operatorname{Sp}(2 N)$ theory. In particular, this analysis implies that the minimal orbit in $C_{N}$ is dual to a rigid semisimple orbit in $B_{N}$, thus explaining a somewhat subtle duality in the case of $B_{2}$ and $C_{2}$ discussed in Section 5.1.

Suppose that a surface operator associated with a nilpotent orbit maps under duality to a surface operator associated with a nilpotent orbit of the dual group. The conjecture implies that $G_{\mathbb{R}}$-representations obtained by quantizing such an orbit have trivial (or integral) central character $\zeta$. These are precisely the special nilpotent orbits (which were described in Section 6.1). Because of this property, they are sometimes called "quantizable" orbits in the mathematical literature, cf. $[8,23]$. In particular, it follows that the pairs of rigid unipotent conjugacy classes in $G_{\mathbb{C}}$ and ${ }^{L} G_{\mathbb{C}}$ which are related to each other by $S$-duality are precisely the special ones. We have checked this prediction for all strongly rigid surface operators in $B_{N}$ and $C_{N}$ up to rank $N=10$, assuming that only strongly rigid conjugacy classes need to be considered in the dual group, and using the invariants we know for surface operators to partly constrain the duality map.

\section{Stringy constructions of rigid surface operators}

\subsection{Holographic description}

For gauge groups of classical types $A, B, C$, or $D$, the large $N$ limit of the $\mathcal{N}=4$ super Yang-Mills theory is believed to be equivalent to type IIB string theory in space-time $\operatorname{AdS}_{5} \times Q$, where the "horizon" $Q$ equals 
$\mathrm{S}^{5}$ if $G=\mathrm{SU}(N)$ and or $\mathbb{R P}^{5}$ if $G$ is an orthogonal or symplectic group $[1,24]$. In particular, under this duality, the superconformal symmetry group $\operatorname{PSU}(2,2 \mid 4)$ of the $\mathcal{N}=4$ gauge theory is identified with the isometry group of the super-geometry whose bosonic reduction is $\operatorname{AdS}_{5} \times Q$. In orientifold models with $Q=\mathbb{R} \mathbb{P}^{5}$, different choices of the gauge group $G$ correspond to different values of the discrete torsion for the two-form fields $B_{N S}$ and $B_{R R}$. Following [24], we introduce discrete holonomies

$$
\theta_{\mathrm{NS}}=\int_{\mathbb{R P}^{2}} \frac{B_{\mathrm{NS}}}{2 \pi} \quad, \quad \theta_{\mathrm{RR}}=\int_{\mathbb{R P}^{2}} \frac{B_{R R}}{2 \pi}
$$

which can take two values, 0 and $\frac{1}{2}$, since $\mathbb{R P}^{2} \subset \mathbb{R P}^{5}$ is a two-torsion element, generating $H_{2}\left(\mathbb{R P}^{5}, \widetilde{\mathbb{Z}}\right)=\mathbb{Z}_{2}$. $(\widetilde{\mathbb{Z}}$ is a twisted version of the constant sheaf of integers.)

A rigid surface operator of a type studied in this paper breaks the fourdimensional conformal group $\mathrm{SU}(2,2) \cong \mathrm{SO}(2,4)$ down to a subgroup $\mathrm{SO}(2,2) \times \mathrm{SO}(2)$. Moreover, just like half-BPS surface operators in [2], it introduces a singularity for two components of the Higgs field and, therefore, breaks the $R$-symmetry group $\mathrm{SO}(6)_{\mathcal{R}}$ down to a subgroup

$$
\mathrm{SO}(6)_{\mathcal{R}} \rightarrow \mathrm{SO}(4) \times \mathrm{SO}(2)
$$

The remaining symmetry group

$$
\mathrm{SO}(2,2) \times \mathrm{SO}(2) \times \mathrm{SO}(4) \times \mathrm{SO}(2)
$$

is precisely the part of the isometry of $\operatorname{AdS}_{5} \times \mathbf{S}^{5}$ preserved by a "probe" D3'-brane embedded in $\mathrm{AdS}_{5} \times Q$ as

$$
\begin{array}{lcll}
\text { space-time: } & \mathrm{AdS}_{5} \times Q \\
\text { D3'-brane: } & \mathrm{AdS}_{3} \times \ell
\end{array}
$$

where $\ell \subset Q$ is "equator" of $Q$.

The identification of the parameters is similar to the $\mathrm{SU}(N)$ case considered in [2]. Thus, if we denote the gauge field on the D3'-brane by $A^{\prime}$, the parameter $\alpha$ is simply the holonomy of $A^{\prime}$, while $\eta$ is identified with the 
holonomy of the dual photon $\widetilde{A}^{\prime}$,

$$
\alpha=\operatorname{Hol}_{\ell}\left(A^{\prime}\right), \quad \eta=\operatorname{Hol}_{\ell}\left(\widetilde{A}^{\prime}\right) .
$$

Similarly, in this description, $\beta$ and $\gamma$ determine the asymptotic behavior of the Higgs field $\varphi^{\prime}$ on the D3'-brane. Namely, it has the familiar form

$$
\varphi^{\prime}=\frac{1}{2 z}(\beta+\mathrm{i} \gamma)+\cdots
$$

where $z$ is a complex variable on the $\mathrm{D} 3^{\prime}$-brane world volume. It is convenient to introduce coordinates $\left(y_{1}, y_{2}, y_{3}, \chi\right)$ on the D3'-brane world volume, $\mathrm{AdS}_{3} \times \ell$, such that the metric takes the standard form

$$
d s^{2}=\frac{1}{y_{3}^{2}}\left(d y_{1}^{2}+d y_{2}^{2}+d y_{3}^{2}\right)+d \chi^{2}
$$

In terms of these coordinates, we have $z=y_{3} \mathrm{e}^{\mathrm{i} \chi}$.

In general, the number of D3'-branes determines the number of independent parameters $\alpha$, as well as $\beta, \gamma$, and $\eta$. (This is clear from the intersecting brane model, which will be discussed below.) In other words, the number of D3'-branes is equal to the number of abelian factors in the Levi subgroup $\mathbb{L}$. For example, in a theory with gauge group $G=\mathrm{SU}(N)$, a single D3'-brane corresponds to a surface operator with maximal Levi subgroup $\mathbb{L}=\mathrm{SU}(N-1) \times U(1)$. In general, larger number of D3'-branes corresponds to surface operators with smaller Levi subgroups and larger orbits $\mathfrak{c} \subset \mathfrak{g}_{\mathbb{C}}$.

The identification of the parameters allows to see how surface operators in this holographic description transform under $S$-duality. Indeed, since $S$ duality in the D3- and D3'-brane theory correspond to $S$-duality in type IIB string theory, it easily follows that $\alpha$ and $\eta$ transform as

$$
S: \quad(\alpha, \eta) \rightarrow(\eta,-\alpha)
$$

while $\beta$ and $\gamma$ are essentially invariant under $S$-duality.

\subsection{Application: $\mathrm{SO}(2 N)$ gauge theory}

In Section 7, we made a general proposal on how $S$-duality should act on surface operators associated with (rigid) unipotent conjugacy classes. Here, we will go in the opposite direction and use the holographic description 
to study the action of $S$-duality on surface operators associated with rigid semisimple conjugacy classes.

Let us consider a simple case of $\mathrm{SO}(2 N)$ gauge theory, whose holographic dual is given by $\mathrm{AdS}_{5} \times \mathbb{R} \mathbb{P}^{5}$ with no discrete torsion, $\left(\theta_{\mathrm{NS}}, \theta_{\mathrm{RR}}\right)=(0,0)$. A particular class of rigid surface operators which is easy to describe in this holographic setup consists of strongly rigid surface operators with semisimple holonomy $V=S$ of the form (2.31),

$$
S=\operatorname{diag}(+1,+1 \ldots,+1, \underbrace{-1,-1, \ldots,-1,-1}_{2 k}), \quad 1<k \leq\left[\frac{N}{2}\right] .
$$

This surface operator corresponds to $k \mathrm{D} 3^{\prime}$-branes with non-trivial holonomy $\alpha=\frac{1}{2}$ and with $\beta=\gamma=\eta=0$. An element $S$ of the form (8.8) breaks the gauge group $G=\mathrm{SO}(2 N)$ down to a subgroup,

$$
G \rightarrow S(O(2 k) \times O(2 N-2 k))
$$

so that we label this surface operator by the conjugacy class,

$$
\mathfrak{C}=\mathfrak{C}_{\left(\left[1^{2 k}\right],\left[1^{2 N-2 k}\right]\right)}^{D_{k} \times D_{N-k}}
$$

Now, let us consider what happens under duality. Since in the D3'-brane theory, $S$-duality exchanges $\alpha$ and $\eta$, just as in (8.7), it follows that strongly rigid surface operators associated with $S \neq 1$ given by (8.8) are mapped to rigid surface operators with ${ }^{L_{\alpha}}={ }^{L_{\beta}}={ }^{L_{\gamma}}=0$ and ${ }^{L_{\eta}} \neq 0$. In particular, such surface operators should correspond to rigid unipotent conjugacy classes ${ }^{{ }^{C}} \subset{ }^{L} G_{\mathbb{C}}$ since they have ${ }^{L} \alpha={ }^{L} \gamma=0$ and, hence, ${ }^{L} S=1$, cf. (2.8). In other words, the holographic description of these surface operators suggests that, under duality, they are mapped to strongly rigid surface operators labeled by rigid unipotent conjugacy classes ${ }^{L_{\mathfrak{C}} \text {, }}$

$$
S: \quad \begin{gathered}
\text { rigid semisimple } \\
(S \neq 1, U=1)
\end{gathered} \longrightarrow \quad \begin{gathered}
\text { rigid unipotent. } \\
\left({ }^{L} S=1,{ }^{L} U \neq 1\right)
\end{gathered}
$$

It is not yet clear how to deduce from this holographic description the dictionary between values of $L_{\eta}$ and the corresponding unipotent conjugacy classes ${ }^{L_{\mathfrak{C}}}$. However, we can determine what the answer must be by comparing invariants described in Section 4. We find that rigid semisimple conjugacy classes (8.9) are dual to rigid unipotent conjugacy classes labeled 
by the partition

$$
\lambda=\left[3,2^{2 k-2}, 1^{2 N-4 k+1}\right] .
$$

It is easy to see that the unipotent conjugacy class labeled by this partition is indeed rigid. Namely, it has no gaps — which, according to Section 2.3, is one of the criteria for rigidness - as long as $1<k \leq\left[\frac{N}{2}\right]$. The second condition for rigidness says that no odd part of $\lambda$ should occur exactly twice. This condition automatically holds true for the partition (8.11) since the only odd parts are " 3 " and " 1 ," and their multiplicities are always odd.

We note that the duality we arrived at, which relates rigid semisimple surface operators with the monodromy (8.8) and rigid unipotent surface operators associated with the conjugacy class labeled by (8.11), is consistent with the general proposal of Section 7 . Indeed, from the relation with quantization discussed in Section 7 it follows that the only rigid unipotent surface operators which under duality are mapped to rigid unipotent operators are those associated with special conjugacy classes. Therefore, as a consistency check, we should verify that the unipotent conjugacy class labeled by partition (8.11) is not special. This is easy to do using the criterion described in Section 6.1. According to this criterion, a unipotent conjugacy class $\mathfrak{C}_{\lambda}$ in $D_{N}$ is special if and only if the transpose partition $\lambda^{t}$ is symplectic. The transpose of the partition (8.11) is

$$
\lambda^{\mathrm{t}}=[2 N-2 k, 2 k-1,1] .
$$

Clearly, this $\lambda^{\mathrm{t}}$ is not symplectic since odd parts " $(2 k-1)$ " and " 1 " have odd multiplicity. Hence, we conclude that the unipotent conjugacy class labeled by partition (8.11) is not special and, therefore, according to the general proposal in Section 7 under duality should transform into a rigid semisimple conjugacy class. This is precisely what we find in this section, from the holographic description of the corresponding rigid surface operators.

Finally, we remark that the duality between surface operators in $\mathrm{SO}(8)$ gauge theory studied in Section 5.2 is a special case of the duality found in this section; it corresponds to $N=4$ and $k=2$.

\subsection{Intersecting brane models}

Now we will reconsider the same subject from the point of view of intersecting brane models. (The holographic models just considered arise from the near-horizon limit of the intersecting brane models.)

In intersecting brane models, gauge theories with symplectic and orthogonal gauge groups can be engineered by introducing orientifold $p$-planes 
(Op-planes). Therefore, let us start by recalling a few basic facts about $O p$-planes, see, e.g., [25]. In type II string theory, an orientifold $p$-plane is defined using a $\mathbb{Z}_{2}$ projection that combines world-sheet orientation symme$\operatorname{try} \Omega$ with a space-time involution $\mathcal{I}_{9-p}$ and, possibly, the action of $(-1)^{F_{L}}$ on fermions,

$$
O p: \quad \mathbb{R}^{1, p} \times \mathbb{R}^{9-p} / \mathcal{I}_{9-p} \Omega \cdot \begin{cases}1, & p=0,1 \bmod 4 \\ (-1)^{F_{L}}, & p=2,3 \bmod 4 .\end{cases}
$$

The action of the orientifold on anti-symmetric tensor fields is given by

$$
\begin{aligned}
& B_{\mathrm{NS}} \rightarrow-B_{\mathrm{NS}}, \\
& C_{p^{\prime}} \rightarrow+C_{p^{\prime}} \quad p^{\prime}=p+1 \bmod 4 \\
& C_{p^{\prime}} \rightarrow-C_{p^{\prime}} \quad p^{\prime}=p+3 \bmod 4 .
\end{aligned}
$$

For $2 \leq p \leq 5$, there are four types of orientifold planes, labeled by the discrete torsion $\left(\theta_{\mathrm{NS}}, \theta_{\mathrm{RR}}\right)$ of the NS-NS three-form field $H$ and of the $(6-$ $p$ )-form field $G_{6-p}$ in the R-R sector, cf. (8.1). We summarize these $O p$ planes, their charges, and the corresponding gauge groups in the table below.

\begin{tabular}{cccc}
\hline$\left(\theta_{\mathrm{NS}}, \theta_{\mathrm{RR}}\right)$ & $\begin{array}{c}\text { Orientifold } \\
\text { plane }\end{array}$ & $G$ & Charge \\
\hline$(0,0)$ & $O p^{-}$ & $\mathrm{SO}(2 N)$ & $-2^{p-5}$ \\
$\left(\frac{1}{2}, 0\right)$ & $O p^{+}$ & $\mathrm{Sp}(2 N)$ & $+2^{p-5}$ \\
$\left(0, \frac{1}{2}\right)$ & $\widetilde{O p}^{-}$ & $\mathrm{SO}(2 N+1)$ & $-2^{p-5}+\frac{1}{2}$ \\
$\left(\frac{1}{2}, \frac{1}{2}\right)$ & $\widetilde{O p}^{+}$ & $\mathrm{Sp}(2 N)$ & $+2^{p-5}$ \\
\hline
\end{tabular}

Maximally supersymmetric $\mathcal{N}=4$ gauge theory with $G=U(N)$ can be realized on the world volume of $N$ D3-branes in type IIB string theory. In this realization, half-BPS surface operators can be obtained by introducing $k$ extra D3'-branes, which intersect D3-branes over the surface $D \subset M$ (figure 4).

Introducing orientifold three-planes on top of the D3-branes leads to stringy realizations of gauge theories with orthogonal and symplectic gauge groups, where the gauge group is determined by the particular type of the O3-plane, according to the above table. On the other hand, the gauge group $G^{\prime}$ on the D3'-branes is a $\mathbb{Z}_{2}$ extension of $U(k)$, which we call $\overline{U(k)}$,

$$
1 \rightarrow U(k) \rightarrow \overline{U(k)} \rightarrow \mathbb{Z}_{2} \rightarrow 1
$$




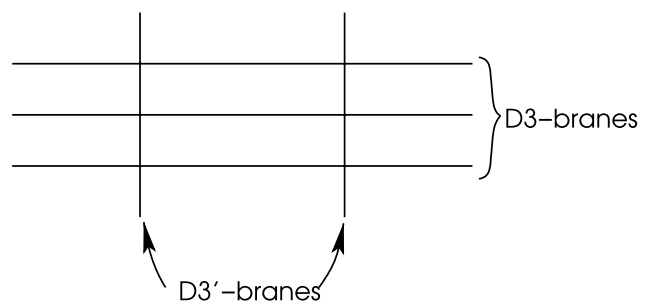

Figure 4: A D-brane realization of surface operators in $U(N)$ gauge theory. $N$ D3-branes (shown horizontally) intersect $k$ extra D3'-branes (shown vertically) over a two-dimensional subspace $D \subset M$.

Specifically, $\overline{U(k)}$ is generated by elements $g_{i}$ of $U(k)$ and the generator $\epsilon$ of $\mathbb{Z}_{2}$, with the commutation relations

$$
\begin{aligned}
& \left(g_{i}, \epsilon\right) \cdot\left(g_{j}, \epsilon\right)=\left(g_{i} \bar{g}_{j}, 1\right), \\
& \left(g_{i}, \epsilon\right) \cdot\left(g_{j}, 1\right)=\left(g_{i} \bar{g}_{j}, \epsilon\right), \\
& \left(g_{i}, 1\right) \cdot\left(g_{j}, \epsilon\right)=\left(g_{i} g_{j}, \epsilon\right) .
\end{aligned}
$$

The holonomy $V^{\prime}=\epsilon$ in the D3'-brane theory breaks $G^{\prime}=\overline{U(k)}$ down to a subgroup, which is the centralizer of $V^{\prime}$ in $G^{\prime}$. For example, $V^{\prime}=\epsilon$ breaks $G^{\prime}$ down to a subgroup $O(k) \times \mathbb{Z}_{2}$. Indeed, it consists of the elements $(g, s) \in$ $G^{\prime}$, such that

$$
(1, \epsilon) \cdot(g, s)=(g, s) \cdot(1, \epsilon)
$$

which implies $g=\bar{g} \in O(k)$.

\subsection{Bubbling geometries}

So far, we discussed stringy description of rigid surface operators in terms D3'-branes realizing $\mathcal{N}=4$ gauge theory either on the world volume of D3branes or via its holographic dual. However, there is yet another, equivalent description, in which D3'-branes are also replaced by a dual geometry. Following [26, 27], we call these bubbling geometries. Conformally invariant half-BPS surface operators in $\mathcal{N}=4$ gauge theory with gauge group $G=\mathrm{SU}(N)$ can be obtained [28] by analytic continuation of the LLM solutions $[26,27]$. These solutions are asymptotic to $\mathrm{AdS}_{5} \times \mathbf{S}^{5}$.

In order to make the symmetry group (8.3) manifest, it is convenient to construct the bubbling geometries as $\mathrm{AdS}_{3} \times \mathbf{S}^{3} \times \mathbf{S}^{1}$ fibrations over the three-dimensional base space $X=\mathbb{R}_{+} \times \mathbb{R}^{2}$ (for more detail, see [28]). In the case of $\mathrm{SU}(N)$ gauge theory, every half-BPS geometry is parametrized by positions $\left(\vec{x}_{i}, y_{i}\right)$ of point "charges" in $X$ of total charge $N$, where $y_{i} \in \mathbb{R}_{\geq 0}$ 


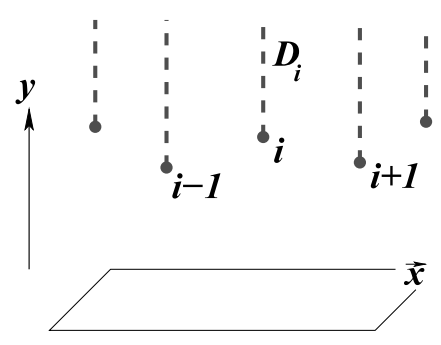

Figure 5: Bubbling geometries are specified by point "charges" in the base space $X=\mathbb{R}_{+} \times \mathbb{R}^{2}$. Semi-infinite dashed lines represent disks $D_{i}$.

and $\vec{x}_{i} \in \mathbb{R}^{2}$ (see figure 5 ). The coordinate $y_{i}$ is related to the value of each charge, $N_{i}$, as

$$
N_{i}=\frac{y_{i}^{2}}{4 \pi l_{p}^{4}}
$$

whereas the coordinate $\vec{x}$ is related to the (eigen-)values of $\beta$ and $\gamma$. Namely, we have ${ }^{18}$

$$
\vec{x}_{i}=\left(\beta_{i}, \gamma_{i}\right) .
$$

In order to describe the geometric interpretation of $\alpha$ and $\eta$, we note that $\mathbf{S}^{1}$ degenerates at every point $\left(\vec{x}_{i}, y_{i}\right)$ (location of the $i$ th charge) and $\mathbf{S}^{3}$ degenerates at the plane $y=0$. Therefore, every bubbling geometry contains some number of five-spheres (one for every point charge in $X$ ) represented by a Hopf-like fibration of $\mathbf{S}^{3} \times \mathbf{S}^{1}$ over the interval $y \in\left[0, y_{i}\right]$, and some number of disks (also, one for every point charge in $X$ ) ending on the asymptotic boundary,

$$
D_{i}=\left\{(y, \chi) \mid y \in\left[y_{i}, \infty\right), \chi \in \mathbf{S}^{1}\right\} .
$$

Here, $\chi$ is the variable parametrizing the $\mathbf{S}^{1}$, as in (8.6). The (eigen-)values of $\alpha$ and $\eta$ are holonimies of the NS and RR two-form fields [28]:

$$
\alpha_{i}=-\int_{D_{i}} \frac{B_{\mathrm{NS}}}{2 \pi} \quad, \quad \eta_{i}=\int_{D_{i}} \frac{B_{\mathrm{RR}}}{2 \pi} .
$$

The $S$-duality of type IIB string theory exchanges $B_{\mathrm{NS}}$ and $B_{\mathrm{RR}}$, thus, providing another evidence for (8.7) to (8.10).

\footnotetext{
${ }^{18}$ In the conventions where $\ell_{s}=1$
} 
For example, the bubbling geometry corresponding to a single charge at $\left(\vec{x}_{0}, y_{0}\right)$, is the familiar space $\mathrm{AdS}_{5} \times \mathbf{S}^{5}$, with the usual metric

$$
d s^{2}=y_{0}\left[\left(\cosh ^{2} u d s_{\mathrm{AdS}_{3}}^{2}+d u^{2}+\sinh ^{2} u d \psi^{2}\right)+\left(\cos ^{2} \theta d \Omega_{3}+d \theta^{2}+\sin ^{2} \theta d \phi^{2}\right)\right],
$$

where the variables are

$$
\begin{aligned}
& x^{1}-x_{0}^{1}+\mathrm{i}\left(x^{2}-x_{0}^{2}\right)=r \mathrm{e}^{\mathrm{i}(\phi-\psi)}, \\
& r=y_{0} \sinh u \sin \theta, \\
& y=y_{0} \cosh u \cos \theta, \\
& \chi=\frac{1}{2}(\phi+\psi) .
\end{aligned}
$$

Now we can extend this construction to describe bubbling geometries representing conformally invariant half-BPS surface operators in $\mathcal{N}=4$ gauge theory with symplectic and orthogonal gauge groups. As usual, this can be achieved by introducing a $\mathbb{Z}_{2}$ orientifold projection, such that the corresponding quotient of the $\operatorname{AdS}_{3} \times \mathbf{S}^{3} \times \mathbf{S}^{1}$ fibrations over $X$ is asymptotic to $\mathrm{AdS}_{5} \times \mathbb{R} \mathbb{P}^{5}$. Since the $\mathbb{Z}_{2}$ involution $\mathcal{I}$ acts trivially on $\mathrm{AdS}_{5}$ and as the antipodal map on $\mathbf{S}^{5}$, it follows from (8.17) to (8.18) that it acts as

$$
\begin{aligned}
\mathbf{S}^{3} & \rightarrow \mathbf{S}^{3} / \mathbb{Z}_{2} \\
\mathcal{I}: \quad \chi & \rightarrow \chi+\frac{\pi}{2} \\
\vec{x} & \rightarrow-\vec{x}
\end{aligned}
$$

Note that $\mathcal{I}$ has no fixed points. Moreover, as usual, the orientifold projection acts non-trivially on the two-form fields $B_{\mathrm{NS}}$ and $B_{\mathrm{RR}}$, cf. (8.13).

The generic surface operator that has deformation parameters $(\alpha, \beta, \gamma, \eta)$ and corresponds to the regular conjugacy class $\mathfrak{C}_{\text {reg }}$ is represented by $N$ pairs of charges at $\pm \vec{x}_{i}$, that is $N$ charges and their "mirror images."

On the other hand, surface operator associated with the rigid semisimple conjugacy class (8.8) and (8.9) is described by the "rigid" configuration with two charges, $N_{1}=2 k$ and $N_{2}=2 N-2 k$, located at $\vec{x}=0$.

\section{Acknowledgments}

We would like to thank R. Bezrukavnikov, A. Braverman, A. Elashvili, D. Gaiotto, V. Kac, G. Lusztig, C. Vafa, and especially D. Kazhdan for 
valuable discussions and correspondence. Research of SG is supported in part by NSF Grant DMS-0635607, in part by RFBR grant 07-02-00645, and in part by the Alfred P. Sloan Foundation. Research of EW is partly supported by NSF Grant PHY-0503584. Conclusions reported here are those of the authors and not of funding agencies.

\section{Appendix A Rigid nilpotent orbits for exceptional groups}

Here we describe rigid nilpotent orbits in exceptional cases. In such cases, the appropriate language to classify nilpotent orbits is based on Bala-Carter theory which we summarize below. According to Bala and Carter, nilpotent orbits in $\mathfrak{g}_{\mathbb{C}}$ are in one-to-one correspondence with pairs $\left(\mathfrak{l}, \mathfrak{p}_{\mathfrak{l}}\right)$, where $\mathfrak{l} \subset \mathfrak{g}$ is a Levi subalgebra, and $\mathfrak{p}_{\mathfrak{l}}$ is a distinguished ${ }^{19}$ parabolic subalgebra of the semisimple algebra $[\mathfrak{l}, \mathfrak{l}]$. Such pairs can be conveniently labeled as $X_{N}\left(a_{i}\right)$ where $X_{N}$ is the Cartan type of the semisimple part of $\mathfrak{l}$ and $i$ is the number of simple roots in any Levi subalgebra of $\mathfrak{p}_{\mathfrak{l}}$. If $i=0$ one simply writes $X_{N}$, and if a simple component of a Levi subalgebra $\mathfrak{l}$ involves short roots (when $\mathfrak{g}$ has two root lengths) then one labels its Cartan type with a tilde. Using this notation, below we list rigid nilpotent orbits in $G_{2}$ :

\begin{tabular}{ccc}
\hline Orbit $\mathfrak{c}$ & $\operatorname{dim}(\mathfrak{c})$ & $\pi_{1}(\mathfrak{c})$ \\
\hline$A_{1}$ & 6 & 1 \\
$\widetilde{A}_{1}$ & 8 & 1 \\
\hline
\end{tabular}

These are the only nilpotent orbits in $G_{2}$ which are not special. As usual, we omit the trivial orbit, and in the last column we also list the $G_{\mathrm{sc}}$-equivariant fundamental group of $\mathfrak{c}\left(\right.$ defined as $\pi_{1}(\mathfrak{c})=G_{\mathrm{sc}}(\mathfrak{c}) / G_{\mathrm{sc}}(\mathfrak{c})^{o}$, where $G_{\mathrm{sc}}(\mathfrak{c})$ is the centralizer of $\mathfrak{c}$ in the simply connected form of $G$ ). The $G_{\text {ad }}$-equivariant fundamental group, usually denoted $A(\mathfrak{c})$, is the same as $\pi_{1}(\mathfrak{c})$ in types $G_{2}$, $F_{4}$, and $E_{8}$.

\footnotetext{
${ }^{19} \mathrm{~A}$ nilpotent orbit in $\mathfrak{g}_{\mathbb{C}}$ is called distinguished if its centralizer contains no semisimple elements which are not in the center of $\mathfrak{g}_{\mathbb{C}}$. In type $A$, the only distinguished orbit is a principal orbit. In types $B, C$, or $D$, an orbit is distinguished if and only if its partition has no repeated parts. Thus, the partition of a distinguished orbit in type $B$ and $D$ has only odd parts, each occurring once, while the partition of a distinguished orbit in type $C$ has only even parts, also occurring only once.
} 
In the following table we list rigid nilpotent orbits in $F_{4}$ :

\begin{tabular}{ccc}
\hline Orbit $\mathfrak{c}$ & $\operatorname{dim}(\mathfrak{c})$ & $\pi_{1}(\mathfrak{c})$ \\
\hline$A_{1}$ & 16 & 1 \\
$\widetilde{A}_{1}$ & 22 & $S_{2}$ \\
$A_{1}+\widetilde{A}_{1}$ & 28 & 1 \\
$A_{2}+\widetilde{A}_{1}$ & 34 & 1 \\
$\widetilde{A}_{2}+A_{1}$ & 36 & 1 \\
\hline
\end{tabular}

All of these orbits, except for $\widetilde{A}_{1}$ and $A_{1}+\widetilde{A}_{1}$, are not special.

In type $E_{6}$, rigid nilpotent orbits are the following:

\begin{tabular}{ccc}
\hline Orbit $\mathfrak{c}$ & $\operatorname{dim}(\mathfrak{c})$ & $\pi_{1}(\mathfrak{c})$ \\
\hline$A_{1}$ & 22 & 1 \\
$3 A_{1}$ & 40 & 1 \\
$2 A_{2}+A_{1}$ & 54 & $\mathbb{Z}_{3}$ \\
\hline
\end{tabular}

The orbit $A_{1}$ is special, while $3 A_{1}$ and $2 A_{2}+A_{1}$ are not. The group $A(\mathfrak{c})$ is trivial for all of these rigid orbits.

In type $E_{7}$, rigid nilpotent orbits are the following:

\begin{tabular}{ccc}
\hline Orbit $\mathfrak{c}$ & $\operatorname{dim}(\mathfrak{c})$ & $\pi_{1}(\mathfrak{c})$ \\
\hline$A_{1}$ & 34 & 1 \\
$2 A_{1}$ & 52 & 1 \\
$\left(3 A_{1}\right)^{\prime}$ & 64 & 1 \\
$4 A_{1}$ & 70 & 1 \\
$A_{2}+2 A_{1}$ & 82 & 1 \\
$2 A_{2}+A_{1}$ & 90 & 1 \\
$\left(A_{3}+A_{1}\right)^{\prime}$ & 92 & 1 \\
\hline
\end{tabular}

All of these orbits have $A(\mathfrak{c})=1$. Among these, the orbits $A_{1}, 2 A_{1}$, and $A_{2}+2 A_{1}$ are special. 
Finally, in the following table we list rigid nilpotent orbits in $E_{8}$ :

\begin{tabular}{ccc}
\hline Orbit $\mathfrak{c}$ & $\operatorname{dim}(\mathfrak{c})$ & $\pi_{1}(\mathfrak{c})$ \\
\hline$A_{1}$ & 58 & 1 \\
$2 A_{1}$ & 92 & 1 \\
$3 A_{1}$ & 112 & 1 \\
$4 A_{1}$ & 128 & 1 \\
$A_{2}+A_{1}$ & 136 & $S_{2}$ \\
$A_{2}+2 A_{1}$ & 146 & 1 \\
$A_{2}+3 A_{1}$ & 154 & 1 \\
$2 A_{2}+A_{1}$ & 162 & 1 \\
$A_{3}+A_{1}$ & 164 & 1 \\
$2 A_{2}+2 A_{1}$ & 168 & 1 \\
$A_{3}+2 A_{1}$ & 172 & 1 \\
$D_{4}\left(a_{1}\right)+A_{1}$ & 176 & $S_{3}$ \\
$A_{3}+A_{2}+A_{1}$ & 182 & 1 \\
$2 A_{3}$ & 188 & 1 \\
$A_{4}+A_{3}$ & 200 & 1 \\
$A_{5}+A_{1}$ & 202 & 1 \\
$D_{5}\left(a_{1}\right)+A_{2}$ & 202 & 1 \\
\hline
\end{tabular}

The only special orbits in this list are $A_{1}, 2 A_{1}, A_{2}+A_{1}, A_{2}+2 A_{1}$, $D_{4}\left(a_{1}\right)+A_{1}$.

\section{Appendix B Orthogonal and symplectic Lie algebras and duality}

In this appendix, we recall the root systems of the Lie algebras $\mathfrak{s o}(2 N+1)$ and $\mathfrak{s p}(2 N)$. In particular, we describe a convenient matrix realization that leads to a simple identification of the invariant polynomials $\operatorname{Tr} \varphi^{k}$ in the corresponding fundamental representations.

We begin with the symplectic group $\operatorname{Sp}(2 N)$. It consists of $(2 N) \times(2 N)$ matrices $A$ that satisfy

$$
A^{t} J A=J
$$

where

$$
J=\left(\begin{array}{cc}
0 & I_{N} \\
-I_{N} & 0
\end{array}\right)
$$


The matrix form of the corresponding Lie algebra, $\mathfrak{s p}(2 N)$, can be obtained by writing $A=\exp (X) \simeq I+X$ in terms of $N \times N$ matrices $X_{i}$,

$$
X=\left(\begin{array}{ll}
X_{1} & X_{2} \\
X_{3} & X_{4}
\end{array}\right)
$$

Then, condition (B.1) implies

$$
X_{1}^{\mathrm{t}}=-X_{4}, \quad X_{2}^{\mathrm{t}}=X_{2}, \quad X_{3}^{\mathrm{t}}=X_{3}
$$

The Lie algebra, $\mathfrak{t}$, of the maximal torus of $\operatorname{Sp}(2 N)$ can be represented by diagonal matrices of the form

$$
X=\left(\begin{array}{cc}
D & 0 \\
0 & -D
\end{array}\right)
$$

where $D=\operatorname{diag}\left(x_{1}, x_{2}, \ldots, x_{N}\right)$.

Now, in this $2 N$-dimensional representation, let us define the root system of $\mathfrak{s p}(2 N)$

$$
\Lambda_{\mathrm{rt}}=\left\{ \pm\left(e_{i} \pm e_{j}\right), 1 \leq i<j \leq N\right\} \cup\left\{ \pm 2 e_{i}, i=1, \ldots, N\right\}
$$

the set of positive roots

$$
\Lambda_{\mathrm{rt}}^{+}=\left\{e_{i} \pm e_{j}, 1 \leq i<j \leq N\right\} \cup\left\{2 e_{i}, i=1, \ldots, N\right\}
$$

and the set of simple roots

$$
\Delta=\left\{e_{i}-e_{i+1}, 1 \leq i<N\right\} \cup\left\{2 e_{N}\right\}
$$

Here, $e_{i}$ denote basis elements of $\mathfrak{t}^{*} \cong \mathbb{R}^{N}$. The $2\left(N^{2}-N\right)$ short roots $\pm e_{i} \pm e_{j}$ can be represented by matrices (see, e.g., [12]):

$$
\begin{aligned}
X_{e_{i}-e_{j}} & =E_{i, j}-E_{j+N, i+N}, \\
X_{e_{i}+e_{j}} & =E_{i, j+N}+E_{j, i+N}, \\
X_{-e_{i}-e_{j}} & =E_{i+N, j}+E_{j+N, i},
\end{aligned}
$$

where $E_{i, j}$ is a matrix with 1 at the position $(i, j)$ and zeros elsewhere. Similarly, $2 N$ long roots $\pm 2 e_{i}$ are represented by matrices

$$
\begin{aligned}
X_{2 e_{i}} & =E_{i, i+N}, \\
X_{-2 e_{i}} & =E_{i+N, i} .
\end{aligned}
$$



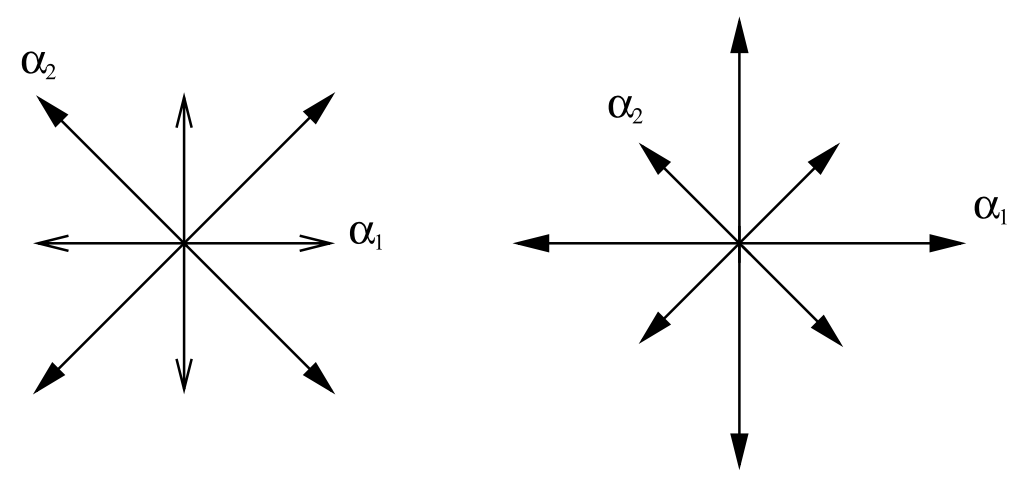

Figure 6: The root systems of type $B_{2}$ and $C_{2}$.

Choosing a metric on $\mathfrak{t}$ defines a natural isomorphism between $\mathfrak{t}$ and $\mathfrak{t}^{*}$ that we need later. We normalize the metric so that short coroots (equivalently, long roots) have length squared 2 . With this normalization, in type $C_{2}$ (see figure 6) we have

$$
\alpha_{1}=\sqrt{2} e_{1}, \quad \alpha_{2}=\frac{1}{\sqrt{2}}\left(e_{2}-e_{1}\right),
$$

where $\left\{e_{1}, e_{2}\right\}$ is an orthonormal basis of $\mathfrak{t}^{*} \cong \mathbb{R}^{2}$.

Now, let us consider the orthogonal group $\mathrm{SO}(2 N+1)$. In the $(2 N+1)$ dimensional representation, it is realized by $(2 N+1) \times(2 N+1)$ matrices $A$ which satisfy

$$
A^{\mathrm{t}} A=I \text {. }
$$

In order to obtain the matrix form of the corresponding Lie algebra $\mathfrak{s o}(2 N+$ 1 ), we write $A=\exp (X) \simeq I+X$. Then, condition (B.8) leads to the following condition on the Lie algebra element $X$;

$$
X+X^{\mathrm{t}}=0 .
$$

In particular, this condition implies that all diagonal elements of $X$ vanish.

Our goal, however, is to describe a matrix realization of the Lie algebra $\mathfrak{s o}(2 N+1)$ which would allow a simple comparison of the invariant polynomials in the dual Lie algebras $\mathfrak{s p}(2 N)$ and $\mathfrak{s o}(2 N+1)$. This will be easy to achieve if we can realize the Cartan subalgebra of $\mathfrak{s o}(2 N+1)$ by diagonal matrices, as we did in equation (B.4) for $\mathfrak{s p}(2 N)$. For this reason, it is 
convenient to perform a unitary transformation on matrices $A$,

$$
A=U B U^{\mathrm{t}}
$$

which after substituting to (B.8) and writing $B=\exp (X) \simeq I+X$ gives a condition on the Lie algebra element $X$,

$$
X^{\mathrm{t}} K+K X=0
$$

with $K=U^{t} U$.

In the $(2 N+1)$-dimensional representation that we are considering, we write matrices $X$ in the block form

$$
X=\left(\begin{array}{ccc}
X_{0} & a & b \\
c & X_{1} & X_{2} \\
d & X_{3} & X_{4}
\end{array}\right)
$$

where the diagonal blocks $X_{0}, X_{1}$, and $X_{4}$ have size $1, N$, and $N$, respectively. In this presentation, we choose

$$
U=\frac{1}{\sqrt{2}}\left(\begin{array}{ccc}
\sqrt{2} & 0 & 0 \\
0 & \mathrm{i} I_{N} & -\mathrm{i} I_{N} \\
0 & -I_{N} & -I_{N}
\end{array}\right)
$$

which gives

$$
K=U^{\mathrm{t}} U=\left(\begin{array}{ccc}
1 & 0 & 0 \\
0 & 0 & I_{N} \\
0 & I_{N} & 0
\end{array}\right)
$$

so that condition (B.9) becomes

$$
\begin{aligned}
& X_{0}=0, \quad X_{1}^{\mathrm{t}}=-X_{4}, \\
& c=-b^{\mathrm{t}}, \quad X_{2}^{\mathrm{t}}=-X_{2}, \\
& d=-a^{\mathrm{t}}, \quad X_{3}^{\mathrm{t}}=-X_{3} .
\end{aligned}
$$

Therefore, in this representations, we can realize elements of the Lie algebra $\mathfrak{s o}(2 N+1)$ by matrices of the form

$$
X=\left(\begin{array}{ccc}
0 & a & b \\
-b^{\mathrm{t}} & X_{1} & X_{2} \\
-a^{\mathrm{t}} & X_{3} & -X_{1}^{\mathrm{t}}
\end{array}\right)
$$

where $X_{1}$ is arbitrary and $X_{2}$ and $X_{3}$ are anti-symmetric. This form is similar to realization (B.2) and (B.3) of the Lie algebra $\mathfrak{s p}(2 N)$. In particular, as in (B.4) the Cartan subalgebra of $\mathfrak{s o}(2 N+1)$ is realized by diagonal 
matrices of the form

$$
X=\left(\begin{array}{ccc}
0 & 0 & 0 \\
0 & D & 0 \\
0 & 0 & -D^{\mathrm{t}}
\end{array}\right)
$$

Now, let us describe the root system of $\mathfrak{s o}(2 N+1)$,

$$
\Lambda_{\mathrm{rt}}=\left\{ \pm\left(e_{i} \pm e_{j}\right), 1 \leq i<j \leq N\right\} \cup\left\{ \pm e_{i}, i=1, \ldots, N\right\}
$$

with the standard choice of positive roots

$$
\Lambda_{\mathrm{rt}}^{+}=\left\{e_{i} \pm e_{j}, 1 \leq i<j \leq N\right\} \cup\left\{e_{i}, i=1, \ldots, N\right\}
$$

and simple roots

$$
\Delta=\left\{e_{i}-e_{i+1}, 1 \leq i<N\right\} \cup\left\{e_{N}\right\} .
$$

In the $(2 N+1)$-dimensional representation (B.10), $2\left(N^{2}-N\right)$ long roots $\pm e_{i} \pm e_{j}$ are represented by matrices

$$
\begin{aligned}
X_{e_{i}-e_{j}} & =E_{i+1, j+1}-E_{j+N+1, i+N+1}, \\
X_{e_{i}+e_{j}} & =E_{i+1, j+N+1}-E_{j+1, i+N+1}, \\
X_{-e_{i}-e_{j}} & =E_{i+N+1, j+1}-E_{j+N+1, i+1}
\end{aligned}
$$

and $2 N$ short roots $\pm e_{i}$ are represented by matrices

$$
\begin{aligned}
X_{e_{i}} & =E_{1, i+N+1}-E_{i+1,1}, \\
X_{-e_{i}} & =E_{1, i+1}-E_{i+N+1,1} .
\end{aligned}
$$

For example, with our choice of normalization, in type $B_{2}$ we have

$$
\alpha_{1}=e_{1}, \quad \alpha_{2}=e_{2}-e_{1} .
$$

Note, coroots of $B_{2}$ are the same as roots of $C_{2}$ scaled by the factor $\sqrt{n_{\mathfrak{g}}}=$ $\sqrt{2}$, and vice versa.

The matrix realizations of the Lie algebras $\mathfrak{s p}(2 N)$ and $\mathfrak{s o}(2 N+1)$ described here have a nice feature that, in both cases, the Cartan subalgebras are realized by the set of diagonal matrices, (B.4) and (B.11), respectively. This defines a natural map from the Cartan subalgebra of these two Lie algebras, in which we simply identify the "eigenvalues" in equations (B.4) and (B.11) (and add an extra "0" in the case of $\mathfrak{s o}(2 N+1)$ ).

In particular, this map between Cartan subalgebras of $\mathfrak{s p}(2 N)$ and $\mathfrak{s o}(2 N+1)$ gives rise to a map from invariant polynomials of $\mathfrak{s p}(2 N)$ to 
invariant polynomials of $\mathfrak{s o}(2 N+1)$, with the property that $\operatorname{Tr} \varphi^{k}$, with the trace in the $2 N$-dimensional representation of $\mathfrak{s p}(2 N)$, maps to $\operatorname{Tr} \varphi^{k}$, with the trace in the $(2 N+1)$-dimensional representation of $\mathfrak{s o}(2 N+1)$.

\section{References}

[1] J. M. Maldacena, The large $N$ limit of superconformal field theories and supergravity, Adv. Theor. Math. Phys. 2 (1998), 231; hep-th/9711200.

[2] S. Gukov and E. Witten, Gauge theory, ramification, and the geometric langlands program, hep-th/0612073.

[3] A. Kapustin and N. Saulina, The algebra of Wilson-'t Hooft operators, arXiv:0710.2097 [hep-th].

[4] O. DeWolfe, D. Freedman and H. Ooguri, Holography and defect conformal field theories, Phys. Rev. D66 (2002), 025009; hep-th/0111135.

[5] E. Witten, Gauge theory and wild ramification, arXiv:0710.0631 [hep-th].

[6] G. Lusztig, A class of representations of a Weyl group, Proc. Kon. Nederl. Akad. A82 (1979), 323.

[7] G. Lusztig, Notes on unipotent classes, Asian J. Math. 1 (1997), 194.

[8] G. Lusztig, Characters of reductive groups over a finite field. Ann. Mathematics Stud., 107, Princeton University Press, Princeton, NJ, 1984.

[9] N. Hitchin, The self-duality equations on a Riemann surface, Proc. London Math. Soc. (3) $5 \mathbf{5}$ (1987), 59.

[10] C. Simpson, Nonabelian Hodge theory, in Proceedings of the I.C.M., Kyoto 1990, Springer-Verlag, 1991, 198-230.

[11] M. Bershadsky, A. Johansen, V. Sadov and C. Vafa, Topological reduction of 4-d SYM to 2-d sigma models, Nucl. Phys. B448 (1995) 166; hep-th/9501096.

[12] D. Collingwood and W. McGovern, Nilpotent orbits in semisimple Lie algebras, Van Nostrand Reinhold Math. Series, New York, 1993.

[13] P. Kronheimer, Instantons and the geometry of the nilpotent variety, J. Differential Geom. 32 (1990), 473.

[14] C. De Concini and V. Kac, Representations of quantum groups at roots of 1: reduction to the exceptional case, Infinite Analysis, Part A, B (Kyoto, 1991), Adv. Ser. Math. Phys. 16 (1992), 141.

[15] P. Kronheimer, A hyper-Kahlerian structure on coadjoint orbits of a semisimple complex group, J. London Math. Soc. 42 (1990), 193. 
[16] D. Kazhdan and G. Lusztig, Fixed point varieties on affine flag manifolds, Isracl J. Math. 62 (1988), 129.

[17] N. Spaltenstein, Order relations on conjugacy classes and the KazhdanLusztig map, Math. Ann. 292 (1992), 281.

[18] G. Lusztig and N. Spaltenstein, Induced unipotent classes, J. London Math. Soc. 19 (1979), 41.

[19] S. Gukov and E. Witten, D-branes and quantization, Adv. Theor. Math. Phys. 13 (2009), 1-73.

[20] A. Kapustin and E. Witten, Electric-magnetic duality and the geometric Langlands program, hep-th/0604151.

[21] R. Brylinski, Geometric quantization of real minimal nilpotent orbits, Differential Geom. Appl. 9 (1998), 5.

[22] A. Braverman and A. Joseph, The minimal realization from deformation theory, J. Algebra 205 (1998), 13.

[23] W. Borho, J.-L. Brylinski and R. MacPherson, Nilpotent orbits, primitive ideals, and characteristic classes, A Geometric Perspective in Ring Theory, Progress in Math., 78, Birkhauser Boston, Inc., Boston, MA, 1989.

[24] E. Witten, Baryons and branes in anti de Sitter apace, JHEP 9807 (1998), 006; arXiv:hep-th/9805112.

[25] O. Bergman, E. G. Gimon and S. Sugimoto, Orientifolds, RR torsion, and K-theory, JHEP 0105 (2001), 047; arXiv:hep-th/0103183.

[26] H. Lin, O. Lunin and J. M. Maldacena, Bubbling AdS space and 1/2 BPS geometries, JHEP 0410 (2004), 025; arXiv: hep-th/0409174.

[27] H. Lin and J. M. Maldacena, Fivebranes from gauge theory, Phys. Rev. D74 (2006), 084014; arXiv: hep-th/0509235.

[28] J. Gomis and S. Matsuura, Bubbling surface operators and S-duality, JHEP 0706 (2007), 025; arXiv:0704.1657 [hep-th]. 
